\title{
Optimal Atmospheric Trajectory for Aero-Gravity Assist
}

\author{
Fayyaz Ahmed Lohar
}

\author{
December 1994
}

\section{Department of Mechanical Engineering McGill University, Montreal}

Dissertation submitted to the Faculty of Graduate Studies and Research of McGill University in partial fulfillment of the requirement of degree of Doctor of Philosophy

Copyright (C)1994 by Lohar, Fayyaz. All rights reserved. 


\section{Abstract}

Planetary gravity assist is used to increase or decrease the heliocentric velocity of the spacecraft. Gravity assist of a planet can be enhanced by an alternative technique called aero-gravity assist (AGA). The latter uses atmospheric maneuvering around the planet to increase the deflection angle of the planetocentric velocity, which leads to an increase in the heliocentric velocity of the spacecraft. In previous studies, very simple calculations were carried out to demonstrate the advantage of AGA. This study presents a realistic mathematical model of aero-gravity assist. Optimal atmospheric trajectory is synthesized such that the heliocentric velocity of the spacecraft is maximized without or with heating rate constraint. Optimization is carried out using Pontryagin's maximum principle.

In the absence of heating rate constraint, the atmospheric trajectory is such that the spacecraft flies mostly at the maximum lift-to-drag ratio in order to minimize the loss of kinetic energy and increase the angle of rotation around the planet. A comparison of AGA for Venus and Mars shows the overall superiority of using Mars over Venus. The results without heating rate constraint also show that the heating rates can be high, which could increase the wall temperature above an acceptable limit. Therefore, a heating rate constraint is imposed on the atmospheric flight for aero-gravity assist. It is noted that the optimal atmopheric maneuvering with a heating rate constraint reduces the heating rates considerably. They also reveal that

optimal AGA with heating rate constraint gives slightly lower heliocentric velocity than optimal AGA without heating rate constraint, particularly when the drag polar based on the Newtonian theory for the hypersonic regime is used in the numerical computations. 
It is also found that for high energy missions (solar flyby and Pluto missions) Marsaero-gravity assist alone will involve very high Earth launch energy and heating rates. Therefore, the potential of an alternative technique of combining Mars-aero-gravity assist with Jupiter-gravity assist for high energy missions is examined. The analysis shows that the use of this technique could reduce Earth launch energy and heating rates significantly.

This study also indicates that the plane of the heliocentric trajectory of the spacecraft can be changed by planetary atmospheric maneuvering while minimizing or maximizing the heliocentric velocity, depending on the specific aim. The results show that the minimization of heliocentric velocity gives larger heliocentric plane change than maximization of heliocentric velocity. Furthermore, the heliocentric plane change achieved by using atmospheric flight is more efficient than the plane change with a thrust impulse. It is also noted that in the case of minimization of the heliocentric velocity, the advantage of AGA over pure propulsive heliocentric plane change increases with the increase in the heliocentric plane change angle. 


\section{Résumé}

L'assistance de la gravité planétaire est utilisée dans le but d'accoroitre ou de diminuer la vitesse héliocentrique du véhicule spatial. Elle peut être améliorée par une autre technique appelée assistance aéro-gravitationnelle (AAG). Cette dernière utilise une manœuvre en atmosphère autour de la planète pour accroitre l'angle de déviation de la vitesse planéto-centrique, ce qui conduit à une augmentation de la vitesse héliocentrique du vaisseau spatial. Dans les études précédentes, de trés simples calculs ont été développés pour montrer l'avantage de l'AAG. Cette étude présente un modèle mathématique réaliste d'assistance aéro-gravitationnelle. La trajectorie atmosphérique optimale est obtenue de façon à ce que la vitesse héliocentrique de l'engin spatial soit maximisée avec ou sans contrainte sur les taux de réchauffement. L'optimisation est effectuée en utilisant le principe de maximisation de Pontryagin.

Dans l'absence d'une contrainte sur les taux de réchauffement, la trajectoire atmosphérique optimal est telle que le vaisseau spatial vole surtout selon un rapport portance-trâinée maximal afin de minimiser la perte d'énergie cinétique et accroitre l'angle de rotation autour de la planéte. Une comparaison d' AAG entre Vénus et Mars montre qu'il est plus efficace d'utiliser Mars que Vénus. On montre ainsi que, dans ce cas, les taux de réchauffement peuvent étre élévés, ce qui pourrait augmenter la température de paroi au dessus du seuil de tolérance. Par conséquence, une contrainte sur les taux de réchauffement est imposée pendant le vol en atmosphére pour l' AAG. Il faut noter que la manouvre atmosphérique optimale avec contrainte sur les taux de réchauffement réduit considérablement les taux de réchauffement. Les résultats montrent également que l' AAG optimale avec contrainte sur les taux de réchauffement donne une vitesse héliocentrique légérement plus faible que l' AAG 
optimal sans contrainte, particulièrment lorsque la polaire de traînée obtenue à partir de la théorie newtonienne en régime hypersonique est utilisée dans les calculs numériques.

Il est également prouvé que pour des missions à haute énergie (surveil solaire ou mission vers Pluton), l'assistance aéro-gravitationnelle de Mars seule impliquera une trés grande énergie pour le lancement depuis la terre, ainsi que des forts taux de réchauffement. Pour ces raisons, la possibilité d'une autre technique combinant l'AAG de Mars avec celle de Jupiter est étudiée dans le cas des missions à haute énergie. L'analyse montre que l'utilisation de cette technique pourrait diminuer de façon significative l'énergie nécessaire au lancement depuis la terre, ainsi que les taux de réchauffements.

Cette étude montre également que le plan de la trajectoire héliocentrique de l'engin spatial peut être changé par des manœuvres dans l'atmosphére planétaire, tout en minimisant ou maximisant la vitesse héliocentrique, suivant les spécifications de la mission. Les résultats montrent que la minimisation de la vitesse héliocentrique donne un changement de plane héliocentrique plus important qu'en maximisant la vitesse héliocentrique. En outre, le changement de plan héliocentrique, obtenu par un vol en atmosphére, est plus efficace que la changement de plan avec une impulsion de propulseur. Il faut également souligner que dans le cas d'une mimimisation de la vitesse héliocentrique, l'avantage de l' AAG sur un changement de plan par propulsion pur est d'autant plus marqué que l'angle du plan héliocentrique á modifier augmente. 


\section{Acknowledgements}

My deepest gratitude goes to my adivsors, Dr. Arun K. Misra and Dr. Dan Mateescu, for their continual support and confidence in me. Their outstanding ability as an educator and their honesty were prime motivator in my studies. Thanks also to the rest of the faculty in the Department of Mechanical Engineering who have helped to shape my education. Thanks also go out to all of my fellow graduate students for their support and friendship. In particular, I would like to thank my parents and my wife, as this thesis could have not been completed without their continual encouragement and support. 


\section{Nomenclature}

Only frequently used symbols are listed. Other symbols used in this thesis are defined during the presentation of the material.

$a=$ semimajor axis

$A=\rho_{o} S r_{o} C_{L}^{*} / 2 m$, a constant

$b=R / r_{0}$

$B=\rho_{r} S R_{r} C_{L}^{*} / 2 m$, a constant

$C=p_{h} / p_{v}$

$C_{D}=\operatorname{drag}$ coefficient

$C_{D_{\circ}}=$ value of $C_{D}$ when $C_{L}=0$

$C_{L}=$ lift coefficient

$C_{L}^{*}=$ value of $C_{L}$ at $(L / D)_{\max }$

$D=$ magnitude of drag force

$e=$ eccentricity of the trajectory

$E^{*}=(L / D)_{\max }$.

$E_{k}=$ kinetic energy

$\mathcal{E}=$ total orbital energy

$g_{p}=$ planetary acceleration due to gravity

$G=$ universal gravitational constant

$h=r / r_{o} ;$ angular momentum

HEO $=$ High Earth Orbit

$H_{T_{1}}=$ hyperbolic path of vehicle before Aero-Gravity Assist

$H_{T_{2}}=$ hyperbolic path of vehicle after Aero-Gravity Assist 
$\mathcal{H}=$ Hamiltonian

$i=$ planetocentric inclination of the trajectory plane to the equatorial plane

$i_{s}=$ heliocentric inclination of the trajectory plane to the ecliptic plane

$J=$ performace index

$K=$ induced drag factor

$L=$ lift force

$L / D=$ lift-to-drag ratio

LEO = Low Earth Orbit

$m=$ vehicle mass

$M=$ Mach number

OTV $=$ Orbital Transfer Vehicle

$p_{j}=$ adjoint variable associated with state $j$

$p_{d}=$ dynamic pressure

$q_{c}=$ convective heating rate

$q_{r}=$ radiative heating rate

$r=$ radial distance from the planet's center

$r_{o}=$ radius of the planet

$r_{p}=$ closest approach or periapsis distance

$r_{r}=$ reference altitude $+r_{0}$

$R=$ radius of planetary spherical atmosphere

$R_{e}=$ Reynolds number

$S=$ effective vehicle surface area normal to the velocity vector

$S_{h}=$ scale height

$t=$ time

$v=$ non-dimensional planetocentric speed $V / \sqrt{\mu / R}$ or $V / \sqrt{R_{r} g_{p}}$

$v_{s}=$ non-dimensional heliocentric speed $V_{s} / \sqrt{\mu / R}$

$v_{\infty}=$ non-dimensional planetocentric speed very far from the planet $V_{\infty} / \sqrt{\mu / R}$

$V=$ planetocentric speed

$V_{r_{s}}=$ radial component of $V_{s}$

$V_{s}=$ heliocentric speed 
$V_{\theta_{s}}=$ transverse component of $V_{s}$

$V_{\infty}=$ planetocentric speed at far away distance from the planet

$\Delta V=$ change in $V_{s}$

$x, y, z=$ Cartesian coordinates after 3-dimensional atmospheric maneuvering

$X, Y, Z=$ Cartesian coordinates before 3-dimensional atmospheric maneuvering

\section{Greek Symbols}

$\alpha=$ angle between $V_{\infty}^{-}$and $V_{p}$

$\alpha_{\mathfrak{a}}=$ angle of attack

$\beta_{s}=$ heliocentric flight path angle

$\gamma=$ planetocentric flight path angle

$\delta=$ deflection angle

$\eta=\rho / \rho_{o}$

$\theta=$ longitude; true anomaly

$\lambda=$ normalized lift control

$\mu=$ Gravitational constant multiplied by the mass of the planet

$\nu=$ kinematic viscosity

$\zeta=\tan ^{-1}\left(V_{r_{s}} / V_{\theta_{s}}\right)$

$\rho=$ density

$\rho_{0}=$ value of $\rho$ at the reference altitude

$\sigma=$ bank angle

$\tau=$ non-dimensional time; $t \sqrt{R_{r} g_{p}} / R_{r}$

$\phi=$ latitude

$\psi$ =heading angle

$\omega=$ angular velocity of rotation of the planet 


\section{Subscripts}

$e=$ value at atmospheric entry

$f=$ value at atmospheric exit; final condition

$o=$ initial condition

$s=$ value at heliocentric or suncentric orbit

1 =value at $H_{T_{1}}$

2 =value at $H_{T_{2}}$

\section{Superscripts}

- =value before AGA

$+=$ value after $\mathrm{AGA}$ 


\section{Contents}

1 Introduction 1

1.1 Previous Research Work .................. 3

1.1.1 Planetary Gravity Assist . . . . . . . . . . . . 3

1.1.2 Aero-Assisted Maneuvers . . . . . . . . . . . . 4

1.2 Scope of the Thesis .................... 7

1.3 Thesis Organization . . . . . . . . . . . . . 9

2 Formulation of the Problem $\quad 11$

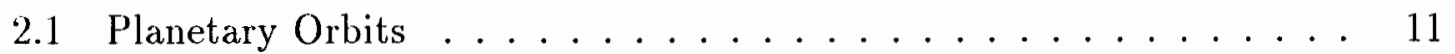

2.2 Patched Conic Method . . . . . . . . . . . . . 13

2.3 Heliocentric Velocity after Gravity Assist . . . . . . . . . . 16

2.4 Equations of Motion . . . . . . . . . . . . . 17

2.4.1 Equations Governing Flight over a Rotating Spherical Planet . 18

2.4.2 Equations Governing Flight over a Non-Rotating Planet . . 20

2.5 Aerodynanic Forces . . . . . . . . . . . . . . . 20

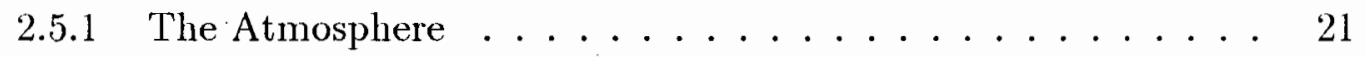

2.5.2 Drag and Lift Forces . . . . . . . . . . . . . . 22

2.5.3 The Drag Polar ................. 23

2.6 Heliocentric Velocity after AGA . . . . . . . . . . . 25

2.6.1 $V_{s}^{+}$after Planar Atmospheric Flight . . . . . . . . 25

2.6.2 $V_{s}^{+}$after 3-Dimensional Atmospheric Flight . . . . . . 26

2.7 Aerodynamic Heating Rate . . . . . . . . . . . . . . 31 
3 Optimization Method 33

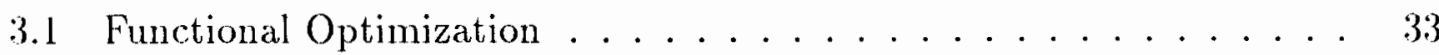

3.1.1 Necessary Conditions for Optimality . . . . . . . . . 34

3.1.2 Transversality Conditions ................ 35

3.2 Method of Solution . . . . . . . . . . . . 36

3.2.1 Shooting Method ............... 37

4 Optimal Aero-Gravity Assist $\quad 39$

4.1 Equations of Motion .................... 39

4.2 Optimization Problem ..................... 40

4.3 Method of Solution . . . . . . . . . . . . . 45

4.4 Validation ...................... 46

4.5 Results and Discussion ... . . . . . . . . . . . . . 48

4.5.1 Comparison of GA with AGA . . . . . . . . . 48

4.5.2 Nature of the Atmospheric Trajectory . . . . . . . . . 51

4.5.3 Effect of $E^{*}$ on Optimal $V_{s}^{+} \ldots \ldots \ldots . \ldots 2$

4.5.4 Effect of $C_{L}^{*}$ on the Heating Rates . . . . . . . . . 54

4.5.5 Comparison of GA and AGA for Mars and Venus . . . . . 56

5 Optimal Aero-Gravity Assist with Heat Constraint 59

5.1 Method of Solution .................... 59

5.1 .1 Inequality Constraints on Heating Rate . . . . . . . . . . . 60

5.1.2 Approach for Solving Optimal AGA Planar Problems with a Heating Rate Constraint ............. 62

5.2 Numerical Results and Discussion . . . . . . . . . . . 63

5.2.1 Nature of the Atmospheric Trajectories without and with Heat Constraint ..................... 64

5.2.2 Comparison of $V_{s}^{+}$without and with Heat Constraint . . . . 69 5.3 Sensitivity Study of Atmspheric Maneuvering with Density Uncertainty 71

6 Aero-Gravity Assist for High Energy Missions $\quad 76$

6.1 Difficulties Associated with Mars-AGA for High Energy Missions . . 77 


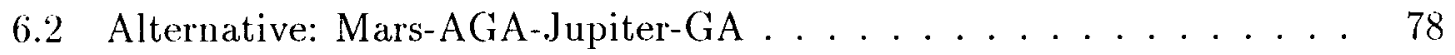

6.2 .1 Travel Time . . . . . . . . . . . . . . 78

6.2 .2 Pluto Mission . . . . . . . . . . . . . . . 81

6.2 .3 Solar Flyby Mission . . . . . . . . . . . . . 83

6.2 .4 Venus-GA-MAGAJGA $\ldots \ldots \ldots \ldots \ldots$

6.3 Discussion . . . . . . . . . . . . . . . 85

7 Optimal Aero-Gravity Assist with Heliocentric Plane Change $\quad 88$

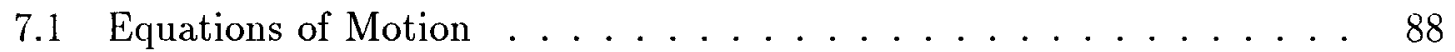

7.2 Variational Formulation $\ldots \ldots \ldots \ldots \ldots$

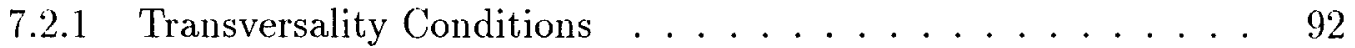

7.3 Method of Solution . . . . . . . . . . . . . . . 94

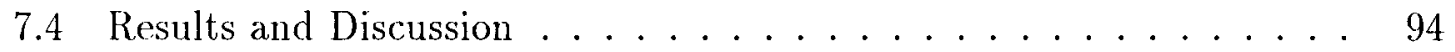

7.4.1 Maximum $V_{s}^{+}$with Heliocentric Plane Change . . . . . 95

7.4.2 Minimum $V_{s}^{+}$with Heliocentric Plane Change . . . . . . 97

7.4.3 Minimization of $V_{s}^{+}$with Different Angles of Heliocentric Plane

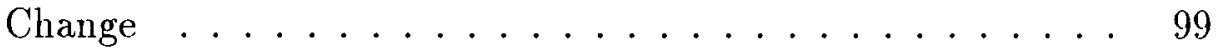

8 Conclusion

A Transversality Conditions for Planar Atmospheric Trajectory

B Transversality Conditions for 3-Dimensional Atmospheric Trajectory 


\section{List of Figures}

1.1 Geometry of planetary flyby trajectory for conventional gravity assist and vector diagram of velocities for gravity assist and aero-gravityassist. Solid and dashed lines show velocities for gravit assist and aerogravity-assist, respectively; superscripts '-' and '+' denote quantities before and after AGA, respectively. . . . . . . . . 4 4

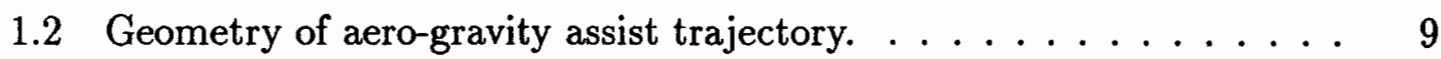

2.1 Vector diagram of velocities for gravity assist or aero-gravity-assist. . 15

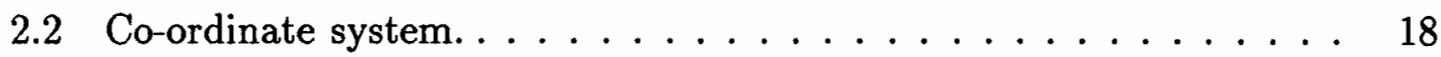

2.3 Aerodynamic forces in the plane of symmetry. . . . . . . . . 22

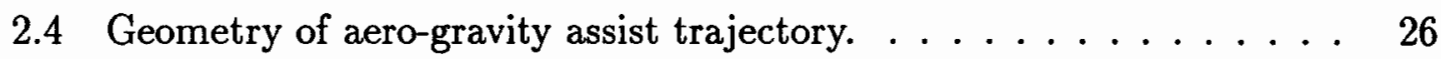

2.5 Velocity vectors, planes, and angles involved in aero-gravity assist with heliocentric plane change $i_{s} \ldots \ldots \ldots \ldots \ldots 27$

2.6 Geometry of trajectory variables and planetocentric orbital elements with respect to the initial plane $O M N \ldots \ldots \ldots \ldots 28$

2.7 Right spherical triangle showing velocity vectors and angles after aero-

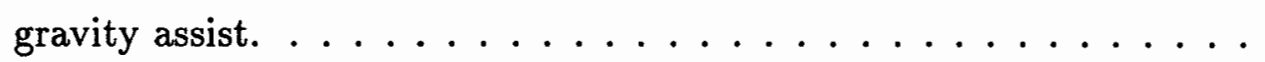

2.8 Right spherical triangles showing heliocentric plane change $i_{s}$, heliocentric flight path angle $\beta_{s}^{+}$, and other angles. . . . . . .

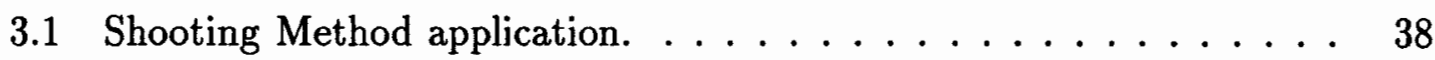

4.1 Comparison of the variation of the state variables with $\theta$ as obtained here with those given by of Mease and Vinh (1985). . . . . . . 47 
4.2 Variation of $V_{s}^{+}$vs $V_{\infty}$ for GA and AGA; o for AGA and solid line for

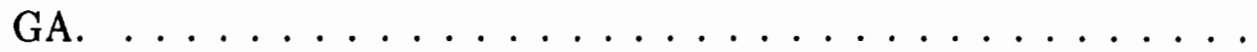

4.3 Variation of the state variables with $\theta$. Solid and dashed lines are for $n=2$ and $n=1.5$, respectively. . . . . . . . . . . .

4.4 Variation of normalized lift coefficient $\lambda$ with $\theta$. Solid and dashed lines are for $n=2$ and $n=1.5$, respectively. . . . . . . . . .

4.5 Variation of $V_{s}^{+}$vs $V_{\infty}^{-}$for GA and AGA for Mars and Venus; o for AGA and solid line for GA. . . . . . . . . . . .

5.1 Variation of the state variables with $\theta$; - without heat constraint; - with heat constraint, $n=2 ;-.-$ with heat constraint, $n=1.5$. . . . .

5.2 Variation of the normalized lift coefficient $\lambda$ and lift-to-drag ratio $L / D$ with $\theta$; - without heat constraint; - with heat constraint, $n=2$; -. with heat constraint, $n=1.5 . \ldots \ldots \ldots$

5.3 Variation of convective heating rate $q_{c}$, dynamic pressure $p_{d}$ and aerolift g-load with $\theta$; - without heat constraint; - - with heat constraint, $n=2 ;-.-$ with heat constraint, $n=1.5 \ldots \ldots 68$

5.4 Variation of altitude with theta from entry; - without heat constraint; - - without heat constraint with overall density $20 \%$ less than that predicted by the exponential model. . . . . . . . . . . .

6.1 Travel time from Mars to Pluto vs $V_{\infty_{M}}^{+}$; - for MAGAJGA or VGAMAGAJGA when $r_{p}=6.0 R_{J}$ at Jupiter; - - for MAGA only, with the perihelion at Mars. . . . . . . . . . . . . 80

6.2 Variation of $V_{\infty_{M}}^{+}$with respect to $V_{\infty}$, after MAGA or VGAMAGA. . 84

$6.3 V_{\infty}$ at Mars after VAGA with $V_{\infty_{E}} \ldots \ldots \ldots \ldots$

7.1 Variation of the normalized lift coefficient $\lambda$ and bank angle $\sigma$ with respect to time; — for $i_{s}=6.00 \mathrm{deg} . ;$ - . for $i_{s}=12.00 \mathrm{deg} . \ldots 102$

7.2 Variation of latitude $\phi$ and heading angle $\psi$ with respect to time; for $i_{s}=6.00 \mathrm{deg} . ;-\ldots$ for $i_{s}=12.00 \mathrm{deg} \ldots \ldots . \ldots 103$ 


\section{List of Tables}

2.1 Orbit Classification . . . . . . . . . . . . . . . 13

4.1 Variation of Venus-AGA Results with $V_{\infty}^{-} \ldots \ldots \ldots \ldots$

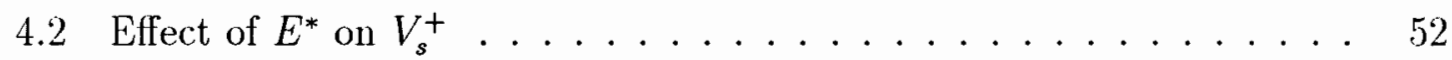

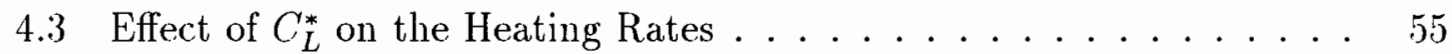

4.4 Comparison of Mars-AGA and Venus-AGA Results with $V_{\infty}^{-} \ldots \ldots 58$

5.1 Variation of AGA Results with $E^{*} \ldots \ldots \ldots \ldots$

5.2 Comparison of some important quantities for AOT and AGA . . . 72

5.3 Effect of density fluctuation on $V_{s}^{+} \ldots \ldots \ldots \ldots \ldots$

6.1 Different quantities related to Pluto Mission with $V_{\infty_{M}}^{+}=14.5 \mathrm{~km} / \mathrm{s} . \quad 78$

6.2 Comparison of JGA, MAGA.JGA and VGAMAGAJGA for a Pluto

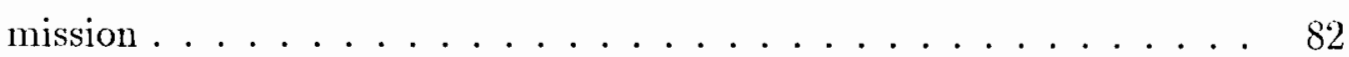

6.3 Potential of JGA, MAGAJGA and VGAMAGAJGA for Solar Missions 85

6.4 Closest approaches of spacecrafts sent for JGA . . . . . . . . 86

7.1 Maximization of $V_{s}^{+}$with $i_{s}=4.5 \mathrm{deg} \ldots \ldots \ldots 96$

7.2 Minimization of $V_{s}^{+}$with $i_{s}=6.0 \mathrm{deg} \ldots \ldots \ldots 98$

7.3 Minimum $V_{s}^{+}$with different $i_{s} \ldots \ldots \ldots \ldots 10 \ldots \ldots$ 


\section{Chapter 1}

\section{Introduction}

Low cost and reduction of transportation time between Earth orbits, as well as that for exploring the solar system, would greatly facilitate the utilization of space. Since mankind entered into the space era, the capability of fast transportation has been limited by the enormous mass of propellant needed to transport payloads in space. This situation has drawn the attention of engineers and scientists towards the development of new techniques for exploiting natural forces so as to minimize the propulsive force. For example, solar sails for propulsion of space vehicles have been proposed to travel within the solar system. Furthermore, the use of "gravity assist", in planetary and solar exploration, has dramatically opened new doors for expanding the set of possible missions for a given launch capability. Another natural force, aerodynamic force, can be used to advantage, as was demonstrated recently in the Magellan mission in which its Venus orbit was altered by utilizing the aero-braking technique. The exploitation of these two natural forces simultaneously, to reduce transportation cost and travel time is the subject of this study.

Planetary gravity assist has already been used several times successfully for exploring the solar system, including the recent Galileo and Ulysses missions, although direct trajectories, i.e., ballistic transfer with no intermediate flyby of any planet has historically been preferred in the trajectory design. The latter results in the shortest flight time to a particular target body, reducing navigation and guidance complexity and operational costs. However, as payload requirements increase, it may be nec- 
essary to use indirect trajectories, such as those using gravity assist, to explore the solar system and other systems beyond it.

The concept of gravity assist is based on the fact that energy can be transferred from or released to a planetary gravity field when a spacecraft's heliocentric trajectory is perturbed by the planet's gravity field. The effect of this perturbation is to rotate the planetocentric velocity vector $V_{\infty}$, thus changing the direction and the magnitude of the spacecraft's heliocentric velocity, $V_{s}$, which is the vector sum of the planet's heliocentric velocity $V_{p}$ and the spacecraft's planetocentric velocity (Kaplan 1976). This phenomenon can be explained by Fig. 1.1a, when the spacecraft passes by the planet with $V_{\infty}$ (planetocentric velocity at sufficiently far away distance; $V_{p}$ in Fig. 1.1a denotes heliocentric velocity of the planet) then the direction of $V_{\infty}$ is changed due to the planet's gravitational pull. Its effect on the heliocentric velocity of the spacecraft can clearly be seen by the vector diagrams, as they show that the heliocentric velocity of the spacecraft is increased and its direction is also changed after planetary flyby. The superscripts '-', ' + ' denote respectively quantities before and after gravity assist. But there are very few massive bodies in the solar system which can cause a sufficient gravitational perturbation to produce a large $\Delta V$ (increase in $V_{s}$ ). As shown in Fig. $1.1 \mathrm{~b}$, large changes in $V_{s}$ are possible only by large $\delta$ (deflection angle due to planetary flyby) or large $V_{\infty}$. Large $V_{\infty}$, on the other hand, needs a large gravity field to undergo a sufficiently large $\delta$, which may not be always available. In that case, multiple-gravity assists have to be used, as Galileo used VEEGA (Venus-Earth-Earth-Gravity-Assist) to reach Jupiter with a comparatively small Earth launch energy (D'Amario, et al. 1989). However VEEGA and similar techniques are unable to take the spacecraft beyond Saturn or to take it to Jupiter with sufficient $V_{\infty}$ so as to obtain Jupiter-gravity-assist (JGA) for solar or Pluto missions. In addition, Jupiter is a remote planet and a large amount of fuel is needed to send the spacecraft there directly to obtain JGA for Pluto or solar flyby missions. Therefore future missions to remote planets (e.g. Neptune and Pluto) or solar flybys require, in addition to JGA, some other novel technique.

One technique which can be quite attractive for future high energy missions is 
aero-gravity assist (AGA), proposed by Randolph and McRonald at JPL (1989). In aero-gravity assist, a spacecraft enters the planetary atmosphere and uses the aerodynamic lift force, in addition to the gravitational pull of the planet, to rotate further around the planet, thereby enhancing the gravity assist on the spacecraft. This is demonstrated in Fig. 1.1b. As one can note due to the additional rotation of $V_{\infty}, V_{s}$ has increased further. To limit the kinetic energy loss due to the aerodynamic drag by the spacecraft during atmospheric maneuvering, it must have a low drag and a high $L / D$ (lift to drag ratio). With possible advent of novel hypersonic vehicles (e.g. waverider), which have high $L / D$, a large deflection angle $\delta$ is possible. This can lead to quite a large $\Delta V$ and change in the direction of $V_{s}$ compared to the simple gravity assist.

\subsection{Previous Research Work}

The research literature relevant to the thesis will be discussed in two parts: (1) research work on gravity assist, (2) studies on aero-assisted maneuvers.

\subsubsection{Planetary Gravity Assist}

Investigators in 1960s proposed the use of gravity assist for exploring the solar system. Hunter (1964) showed the usefulness of Jupiter gravity assist in detail, including solar flyby, 90 deg out-of-the ecliptic flight and Pluto missions. He also pointed out that if smart guidance techniques are used, then gravity assist would open new doors to unmanned exploration of the solar system.

Niehoff (1966) considered several planets for gravity assist, for example, Mars, Venus, Saturn, etc. Dearwester (1966) was the first to suggest multiple gravity assists of outer planets for remote planet missions, such as Jupiter-Saturn-Uranus gravity assist to reach Neptune.

Later, these ideas were transformed into real missions. The Mariner in 1973 used Venus gravity assist to reduce its perihelion and set up a series of Mercury encounters. Poineer 10 and 11 were launched in 1973 and used Jupiter gravity assist for escaping 


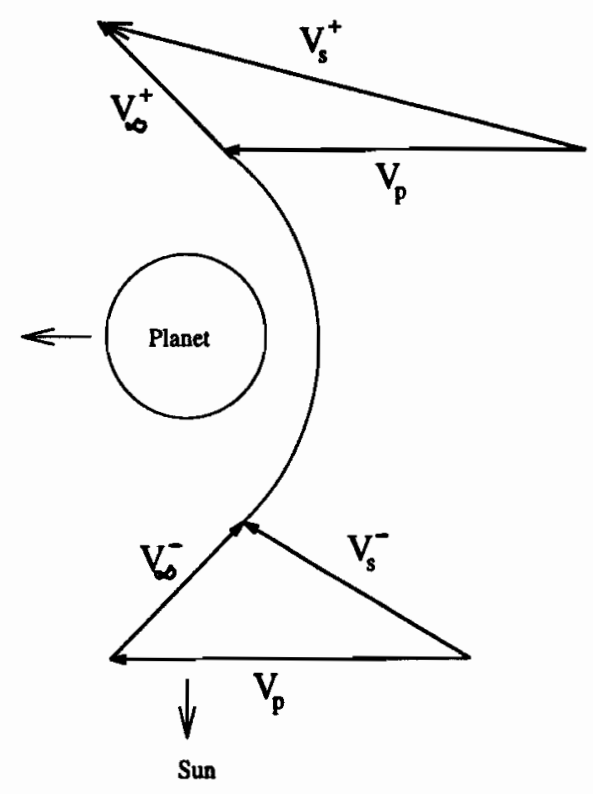

(a)

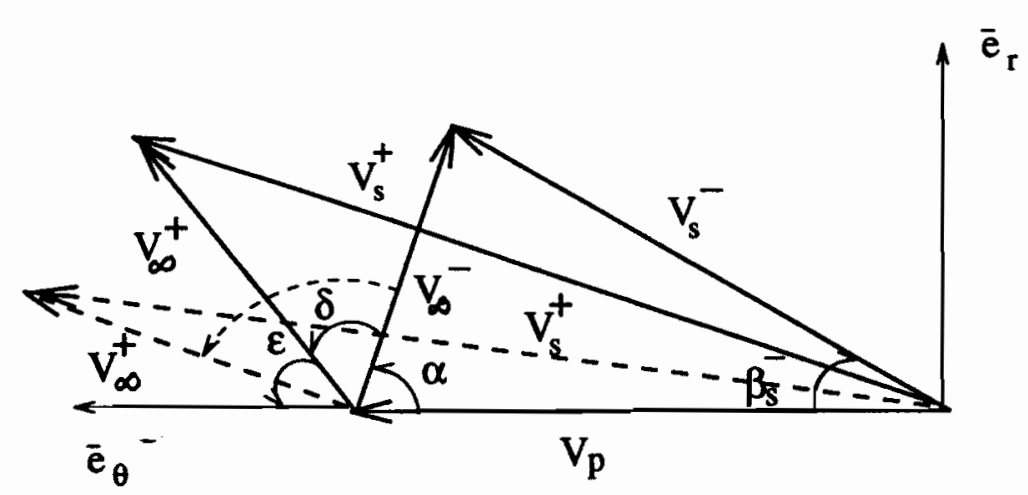

(b)

Figure 1.1: Geometry of planetary flyby trajectory for conventional gravity assist and vector diagram of velocities for gravity assist and aero-gravity-assist. Solid and dashed lines show velocities for gravit assist and aero-gravity-assist, respectively; superscripts '-' and ' + ' denote quantities before and after AGA, respectively. 
from the solar system. Voyager 2 also used Jupiter gravity assist in 1979 to flyby Saturn and Uranus (Rycroft 1990). The Galileo spacecraft was supposed to be launched in 1986 for a direct trajectory to Jupiter. But the Challenger accident caused a temporary halt to the mission and led to intensive research to find new mission options. Finally Galileo was launched in 1989 to reach Jupiter using VEEGA (Venus-EarthEarth-Gravity-Assist) trajectory designed by Diehl et al. (1987) with a relatively small Earth launch energy compared to the direct trajectory (for a comparison, see D'Amario, Byrnes, Johannesen, and Nolan 1989). This technique signalled the beginning of a new era of reducing Earth launch energy for solar and outer planetary flyby missions. Similar reduction is also one of the objectives of this investigation.

\subsubsection{Aero-Assisted Maneuvers}

In 1962 London presented a paper in which he demonstrated the possibility of using atmospheric maneuvering to produce appropriate aerodynamic force so as to change the spacecraft's terrestrial orbit with significantly smaller propellant expenditure compared to an extra-atmospheric propulsive maneuver. Cuadra and Arther (1966) went further to suggest the use of aero-cruise, which means using thrust to cancel the drag during aerodynamic maneuvering, to change the orbital plane of the spacecraft. Since then, several studies on aero-assisted missions have been conducted; these are: synergic plane change, aero-capture, multi-pass aero-braking and - aero-assisted orbit transfer. An excellent survey of investigations on aero-assisted techniques has been given by Walberg (1985). A brief description of these missions will be given below.

Synergic plane change: This is a maneuver in which a change in the orbit inclination is accomplished through a combination of propulsive and aerodynamic forces by giving an appropriate bank angle rather than only by propulsion. The maneuver is applicable to orbits about any planet with a suitable atmosphere.

Aero-capture: In this technique, the velocity reduction is achieved in a single deep atmospheric pass sufficient to transfer the vehicle from its hyperbolic approach trajectory to a desired elliptic orbit about the planet (i.e., the vehicle is captured by 
the planet's gravitational field).

Multi-pass aero-braking: As the name suggests, in this technique several atmospheric entries are required for capture or lowering the orbit (e.g. Magellan mission in 1993). Unlike aero-capture, it is carried out at such high altitudes that nearly free molecular flow is experienced.

Aero-assisted orbit transfer (AOT): In this technique, terrestrial obits are changed using aerodynamic lift and drag forces. There are two kinds of AOT, coplanar and non-coplanar. In the coplanar case, the orbit is changed from a High-Earth-Orbit to a Low-Earth-Orbit by using the aerodynamic drag to decrease the velocity of the spacecraft and using the aerodynamic lift to keep it in the atmosphere until conditions necessary to achieve the desired orbit transfer are satisfied. In non-coplanar AOT, either the orbital plane alone is changed, or both orbit and its plane are changed in a complex maneuver.

Since AOT is very much related to AGA to be studied in this thesis, and since our investigation is inspired by the previous research on AOT, a brief literature survey on it is given below. This literature survey can be divided into two categories: (1) aero-glide, (2) aero-cruise. In aero-glide, no thrust is used during the atmospheric flight. As mentioned earlier, in aero-cruise, thrust is used to cancel the drag during the atmospheric flight.

\section{Aero-Glide}

A fundamental study on coplanar aero-assisted orbit transfer was carried out by Mease and Vinh (1985), in which optimal aerodynamic maneuvering through the atmosphere was used for changing circular orbits from a High-Earth-Orbit (HEO) to a Low-Earth-Orbit (LEO) without and with a heating rate constraint. An excellent monograph by Vinh also deals with this topic (1981). Vinh and Hanson (1985) were the first to analyze aero-assisted orbital plane change; they used calculus of variations to determine optimally varying aerodynamic lift and showed the superiority of AOT over all propulsive modes when the ratio of the initial and final terrestrial orbit radii is between 1 and 4. Miele, Basapur and Mease (1986) proposed a nearly grazing 
planets, Earth, Venus and Mars, and showed that Mars will be a more appropriate planet than Venus for AGA. Later, Lewis and Kothari (1989) developed an analytical model to analyse AGA and presented some results for the Martian atmosphere and gravity field. Anderson, et al. (1992) developed several vehicle configurations for possible use in AGA. Lewis and Kothari (1990) developed some additional analytical models to study AGA and suggested that aerodynamic maneuvering could be used for changing the plane of the spacecraft trajectory and get out of the ecliptic plane. Recently, Lewis and McRonald (1992) used analytical models developed for AGA using various $L / D$ ratios and like Randolph and McRonald (1992) concluded that AGA can be used for a Pluto mission even with the present technology.

\subsection{Scope of the Thesis}

All the studies on AGA cited above are restricted to simple analytical models which were based on several assumptions. For example, in the lower region of the atmospheric trajectory of the spacecraft a circular path at a given altitude was used to increase deflection angle $\delta$. It was also assumed that the drag will be negligible during the time interval between entering the sensible atmosphere and arriving at the chosen altitude of the circular path for aerodynamic maneuvering as well as between the chosen altitude and the atmospheric exit. Furthermore, the $L / D$ ratio of the spacecraft during its maneuvering along the circular path was assumed to be constant. Moreover, aerodynamic heating during maneuvering through the atmosphere was not considered in these studies.

Therefore, the objectives of this thesis are to develop more realistic models and to use calculus of variation techniques to determine optimal atmospheric trajectories for AGA. The optimization problem is solved by using the Hamiltonian formulation based on Pontryagin's maximum principle. The analysis will also be extended to the case when a heat constraint has been imposed. Optimization of the global trajectory, that is, the trajectory from the Earth to the planets for AGA and GA and to the solar system target, will be carried out in such a way that heating rate during AGA is 
low and the Earth launch energy is a minimum. An optimal atmospheric trajectory is determined such that the heliocentric velocity $V$, will be maximized or minimized after AGA. The change of heliocentric orbital plane will also be considered.

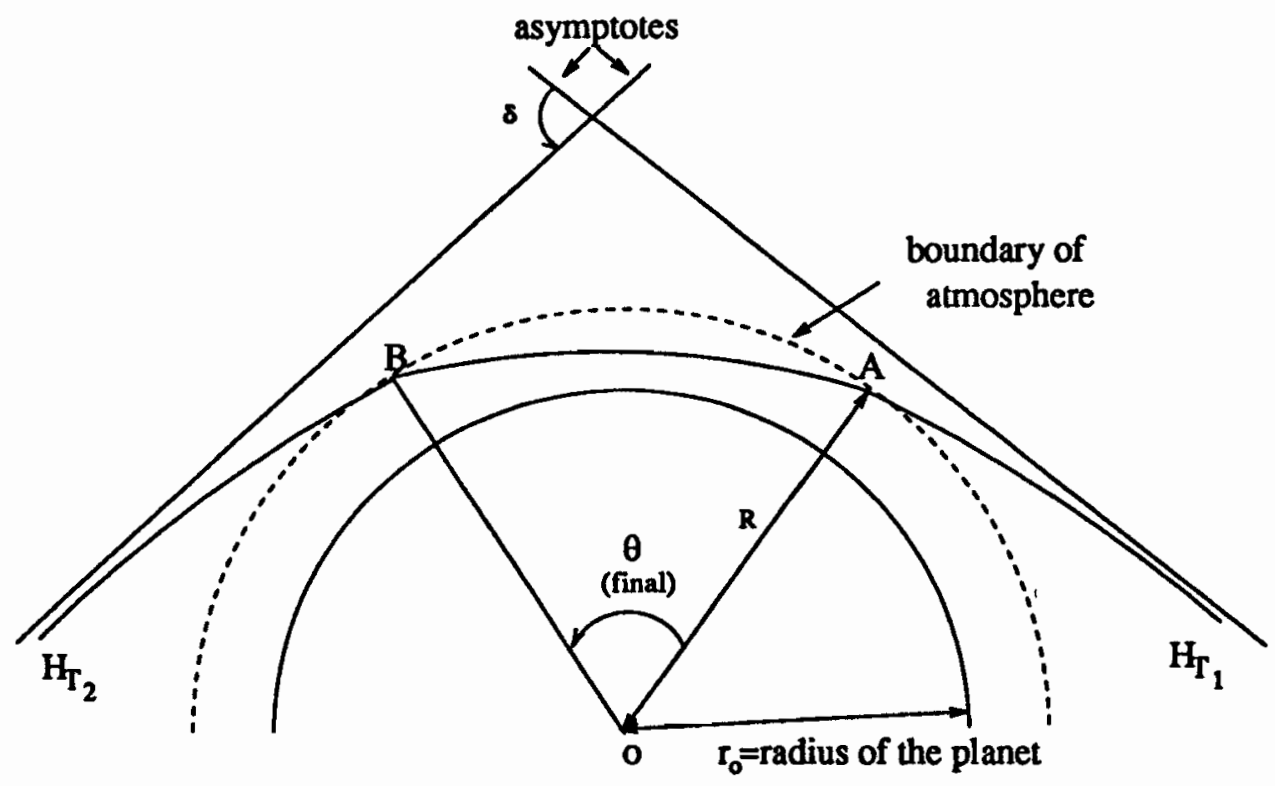

Figure 1.2: Geometry of aero-gravity assist trajectory.

According to the approach used in this thesis, the basic events of an aero-gravityassist mission are as follows. The spacecraft is launched from the Earth orbit with a tangential thrust in the direction of the orbital velocity which injects the vehicle in a heliocentric elliptic transfer orbit in such a way that it arrives at the planet with planetocentric velocity $V_{\infty}^{-}\left(V_{\infty}\right.$ before AGA or GA, where $V_{\infty}$ stands for velocity of the spacecraft at sufficiently far away distance from the planet). The spacecraft approaches the planet along a hyperbolic trajectory $H_{T_{1}}$ with its periapsis located inside the atmosphere of the planet. At point $\mathrm{A}$ in Fig 1.2, the vehicle enters the atmosphere. It flies along an optimal trajectory with minimum loss of kinetic energy, using modulated lift for maneuvering. In case of AGA with heat constraint, the heating rate at the stagnation region should not exceed the prescribed heating rate constraint during atmospheric maneuvering. When it exits from the atmosphere (at point $B$ ), the direction of the path has changed by a large angle $\delta$, while due to the atmospheric drag $V_{\infty}^{+}\left(V_{\infty}\right.$ after AGA) has decreased a little (i.e., $\left.V_{\infty}^{+}<V_{\infty}^{-}\right)$. The spacecraft now follows a new hyperbolic trajectory $H_{T_{2}}$. The flight path angle, angle 
of rotation of the spacecraft through the atmosphere and planetocentric velocity at $B$ are such as to give optimum results. The optimum results can be of several types, e.g. either maximum or minimum $V_{s}$, specific heliocentric flight path angle, etc., at a point on $H_{T_{2}}$ sufficiently far away from the planet.

\subsection{Thesis Organization}

In Chapter 2 the equations of motion and models for calculating aerodynamic heating rates will be presented. In this chapter, the expressions for the objective functions, that is the heliocentric velocity of the spacecraft after AGA for planar and threedimensional atmospheric maneuvering will be derived, which will be used later in optimizing the AGA trajectory.

Chapter 3 will discuss the optimization method to be used in this thesis. The method of solution of the optimization problem will also be discussed.

Chapter 4 will present the results of the analysis regarding optimal planar atmospheric trajectory for aero-gravity assist. A comparison between aero-gravity assist and gravity assist will also be presented. Furthermore, Mars-AGA and Venus-AGA will be compared in detail.

Chapter 5 will discuss optimal atmospheric trajectory for aero-gravity assist with a heat constraint. The effect of fluctuation of the atmospheric density on optimal AGA will also be studied.

In Chapter 6, the problems associated with the use of AGA for planetary and solar missions will be investigated.

In Chapter 7, the heliocentric velocity after AGA, $V_{s}^{+}$, when the heliocentric plane of the spacecraft is changed, will be extremized.

Chapter 8 will present the conclusion of this study, suggestions for further study, and contributions of this investigation to the advancement of knowledge. 


\section{Chapter 2}

\section{Formulation of the Problem}

We have seen in Chapter 1 that an AGA trajectory has three components: (1) a hyperbolic trajectory before AGA; (2) an atmospheric trajectory and (3) a hyperbolic trajectory after AGA. The effect of AGA can be optimized by controlling the atmospheric trajectory since the nature of the hyperbolic trajectory after AGA depends on it. In this chapter, we will formulate the problem in such a way that the effects of all three components of AGA trajectory are combined in one model so that an optimization technique could be applied later to get the optimum benefit of AGA. Firstly, we will present the equations of motion of a spacecraft in a planetary atmo-

sphere. Then, the mathematical models to calculate the heliocentric velocity $V_{s}^{+}$of the spacecraft after planar or three-dimensional atmospheric flight will be developed. Finally, we will discuss the aerodynamic heating rate models, that will be used in our analysis.

For the sake of completeness, it will be appropriate to present a brief introduction to planetary orbits, as two of the component trajectories are in space, and give definitions of the basic terms related to interplanetary trajectory and conventional gravity assist before starting to formulate the problem. 


\subsection{Planetary Orbits}

For all practical orbit situations involving man-made spacecraft, or natural bodies such as a planet or the sun, one of the masses in the two-body system is much greater than the other. Thus if $m_{1} \gg m_{2}$, then the motion of $m_{2}$ about $m_{1}$ is essentially the motion of a particle in an inertially fixed field subjected to the gravitational pull of $m_{1}$. This type of motion is called central force motion. The force of attraction is always directed to a fixed point in the inertial space, with the magnitude solely a function of the distance between the point of interest and the center of attraction. The motion of the spacecraft around the natural body can be easily obtained by solving the two-body problem based on Newtonian inverse-square attraction law. We have,

$$
\frac{d^{2} \boldsymbol{r}}{d t^{2}}+\frac{\mu \boldsymbol{r}}{r^{3}}=0
$$

where $\mu=G\left(m_{1}+m_{2}\right), G$ is the universal gravitational constant and $\boldsymbol{r}$ is the position vector of the orbiting body relative to the central body. Solving Eq. (2.1), one obtains the general polar equation for a conic section with the origin at one of the foci (Kaplan 1976):

$$
r=\frac{h^{2} / \mu}{1+e \cos \theta}
$$

where $\theta$ is the true anomaly, measured from the position where $r$ has the minimum value, $h$ is the angular momentum per unit mass of the orbiting body and is given by $h=r^{2} \dot{\theta}$, while $e$ is eccentricity of the conic section.

The total energy per unit mass of the orbiting body is given by

$$
\mathcal{E}=E_{k}-U
$$

where $E_{k}$ is the kinetic energy of the orbiting body, while $U=\mu / r$ represents the gravitational potential (which is negative potential energy). Allowing $m_{1}$ to be much larger than $m_{2}$ leads to $\mu=G m_{1}$ and permits the kinetic energy expression to become

$$
E_{k}=\frac{1}{2} m_{2} \dot{r} \cdot \dot{r}
$$

$\dot{r}$ in Eq. (2.4) can be written as

$$
\dot{\boldsymbol{r}}=\dot{\boldsymbol{r}} \dot{i}_{r}+r \dot{\theta} \dot{\boldsymbol{i}}_{\theta} \text { or } V=\left(\dot{r}^{2}+r^{2} \dot{\theta}^{2}\right)^{1 / 2} .
$$


Table 2.1: Orbit Classification

\begin{tabular}{|c|l|c|}
\hline Range of $e$ & Orbit Shape & $\mathcal{E}$ \\
\hline$e=0$ & Circle & $-\mu / 2 a<0$ \\
$0<e<1$ & Ellipse & $-\mu / 2 a<0$ \\
$e=1$ & Parabola & 0 \\
$e>1$ & Hyperbola & $-\mu / 2 a>0$ \\
\hline
\end{tabular}

where $V$ is the speed of the spacecraft. Therefore the kinetic energy for unit mass is given by

$$
E_{k}=\frac{1}{2}\left(\dot{r}^{2}+r^{2} \dot{\theta}^{2}\right)
$$

Hence the total energy per unit mass becomes

$$
\mathcal{E}=\frac{V^{2}}{2}-\frac{\mu}{r}=\frac{1}{2}\left(\dot{r}^{2}+r^{2} \dot{\theta}^{2}\right)-\frac{\mu}{r} .
$$

Table 2.1 lists the total energy for various conic section trajectories. When a spacecraft flies by a planet for gravity assist, it has a hyperbolic trajectory. The total energy $\mathcal{E}$ in this case is positive. This allows the spacecraft to have a nonzero planetocentric velocity $V_{\infty}$ far away from the planet. As can be seen from Eq. (2.7), when $r \rightarrow \infty, \mathcal{E}=V_{\infty}{ }^{2} / 2$; equating this to the expression given in Table 2.1, one has $a=-\mu / V_{\infty}^{2}$.

For simplicity, we will use the patched conic method for interplanetary transfer which is briefly described in the next section.

\subsection{Patched Conic Method}

This approach permits us to use the results of the two-body problem to approximate the trajectory of a spacecraft between two attracting bodies, which in reality is a three-body-system. For interplanetary probes, the trajectory has three segments: Earth escape, heliocentric transfer, and planetary encounter. The sequence of calculations consists of ignoring all attracting bodies except the one whose influence is 
the greatest. As the spacecraft leaves a low-Earth-orbit, it will be essentially in a two-body hyperbolic escape trajectory with respect to the Earth. At a certain point, the gravitational sphere of influence of the Earth ends when the sun becomes the primary attracting body. At that point the Earth is ignored, and the spacecraft is in a heliocentric transfer orbit until the target planet is approached. The probe then enters another two-body trajectory about the planet that is being used for gravity assist. Obviously, the sun influences the motion continuously but we ignore it to get an approximate solution. This approach permits quick calculations with sufficient accuracy to conduct preliminary studies (Kaplan 1976).

Now consider an interplanetary transfer from the Earth. The order of calculations is as follows:

(1) Establish the required velocity impulse to escape low-Earth-orbit with a hyperbolic trajectory so that the vehicle trajectory will reach the target planet with some $V_{\infty}^{-}$. A single velocity increment $\Delta V_{E}$ is assumed to start the transfer mission. We have

$$
\Delta V_{E}=V_{p_{E}}-V_{c}
$$

where $V_{p_{E}}$ is the velocity of the spacecraft required at the perigee for the hyperbolic escape, while $V_{c}$ is the orbital speed at the parking orbit. The planetocentric velocity far away from the Earth, $V_{\infty_{E}}$, for the hyperbolic trajectory is given by

$$
V_{\infty_{E}}=\left(V_{p_{E}}^{2}-V_{s c}^{2}\right)^{\frac{1}{2}}
$$

where $V_{s c}$ is the velocity for parabolic escape from the low-Earth-orbit, while $V_{\infty_{B}}$ is called the hyperbolic excess velocity.

(2) The Earth is turned off and the sun takes over. This is the heliocentric phase of the flight. Initial velocity and directions for this phase are transferred from the low-Earth-obit, and starting position is at the Earth. If the angular momentum $h_{s}^{-}$ (it is constant for a specific unperturbed trajectory) of the spacecraft is less than that of the Earth then the heliocentric position of the spacecraft near the Earth is its aphelion; otherwise it is the perihelion. The value of the heliocentric eccentricity $e_{s}^{-}$ 
depends on the heliocentric position of the spacecraft near the Earth and is given by

$$
e_{s}^{-}=\left(h_{s}^{-}\right)^{2} / \mu_{s} r_{E} \pm 1,
$$

where $r_{E}$ is the distance of the Earth from the sun (1 A.U.) and the subscript $s$

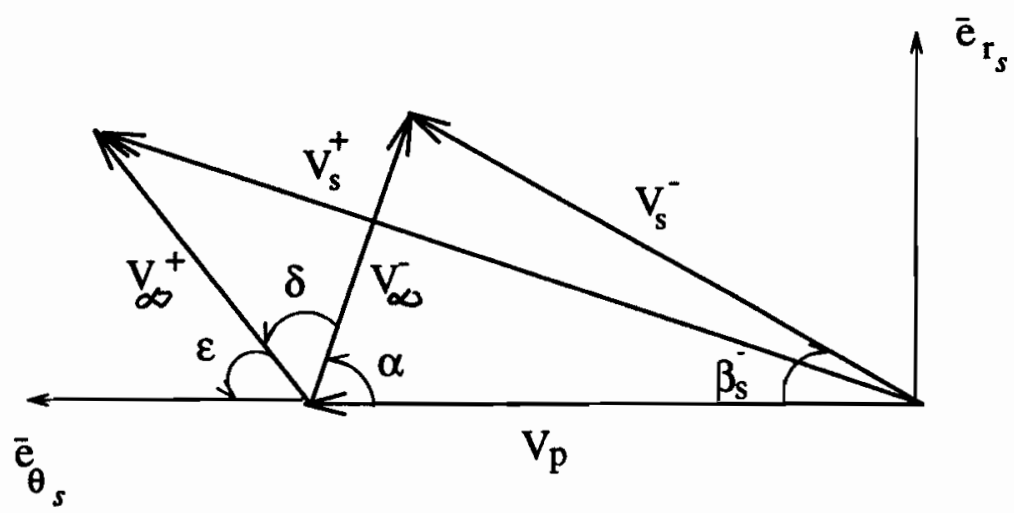

Figure 2.1: Vector diagram of velocities for gravity assist or aero-gravity-assist.

implies that the quantities are related to the sun-centric (heliocentric) trajectory. When the heliocentric position of the spacecraft near the Earth is the aphelion, then the sign is positive otherwise it is negative.

(3) When the target planet is approached, the sun is turned off, and the planetocentric velocity vector $V_{\infty}^{-}$at that point, shown in Fig. 2.1, generates the initial conditions for the hyperbolic passage. In Fig. 2.1, $V_{p}$ is the heliocentric velocity of the planet, while $V_{s}^{-}$is that of the spacecraft prior to the planetary flyby. After the flyby, the planetocentric velocity of the spacecraft has rotated through an angle $\delta$ and its heliocentric velocity becomes $V_{s}^{+}$.

From Fig. 2.1 we have

$$
V_{\infty}^{-}=\left[V_{p}^{2}-2 V_{s}^{-} \cos \beta_{s}^{-} V_{p}+\left(V_{s}^{-}\right)^{2}\right]^{\frac{1}{2}},
$$

where $V_{s}^{-}=h_{s}^{-} \cos \beta_{s}^{-} / r_{s}$ while $\beta_{s}^{-}$is the corresponding flight path angle. The latter is given by

$$
\beta_{s}^{-}=\tan ^{-1}\left[\frac{r_{s} \mu_{s} e_{s}^{-} \sin \theta_{s}^{-}}{\left(h_{s}^{-}\right)^{2}}\right],
$$

where $\theta_{s}^{-}$is the heliocentric true anomaly of the spacecraft at the location of the planet along the interplanetary trajectory and is given by

$$
\theta_{s}^{-}=\cos ^{-1}\left[\frac{1}{e_{s}^{-}}\left\{\frac{\left(h_{s}^{-}\right)^{2}}{r_{s} \mu}-1\right\}\right] .
$$


Figure 2.1 shows the angle between $V_{\infty}^{-}$and $V_{p}$, which can be calculated from

$$
\alpha=\sin ^{-1}\left(\frac{V_{s}^{-} \sin \beta_{s}^{-}}{V_{\infty}^{-}}\right) .
$$

If we know only $V_{\infty}^{-}$then $V_{s}^{-}, \beta_{s}^{-}$and $\Delta V_{E}$ can be determine by iteration.

(4) In the third component trajectory, the planet's gravity field takes over with the above-mentioned conditions at a large distance away from the planet.

\subsection{Heliocentric Velocity after Gravity Assist}

In the case of a hyperbolic orbit, the energy of the spacecraft is given by

$$
\mathcal{E}=\frac{V^{2}}{2}-\frac{\mu}{r}=\frac{V_{\infty}^{2}}{2},
$$

where $V$ is the planetocentric velocity of the spacecraft in the vicinity of the planet. Thus

$$
V_{\infty}=\left(V^{2}-\frac{2 \mu}{r}\right)^{1 / 2} .
$$

For conventional gravity assist, $V_{\infty}^{-}=V_{\infty}^{+}$, but for aero-gravity assist $V_{\infty}^{+}<V_{\infty}^{-}$, because of the loss of kinetic energy due to aerodynamic drag. From Fig. 2.1, the heliocentric velocity $V_{s}^{+}$after either aero-gravity assist or conventional gravity assist is given by

$$
V_{s}^{+}=\left(V_{\theta_{s}}^{2}+V_{r_{s}}^{2}\right)^{1 / 2},
$$

where

$$
V_{\theta_{s}}=V_{p}+V_{\infty}^{+} \cos \epsilon, V_{r_{s}}=V_{\infty}^{+} \sin \epsilon, \epsilon=\pi-\alpha-\delta .
$$

The angle $\alpha$ is defined earlier [Eq. (2.14)], while $\delta$, the bending angle of $V_{\infty}$, depends on the nature of the planetocentric trajectory.

For conventional gravity assist $\delta$ is given by (Kaplan 1976)

$$
\delta=2 \sin ^{-1}\left(\frac{1}{e}\right)
$$

while

$$
e=1+\frac{V_{\infty}^{2} r_{p}}{\mu}
$$


where $r_{p}$ is the closest approach. The value of $\delta$ can be increased by an appropriate atmospheric trajectory in AGA.

In the case as shown in Fig. 2.1, $V_{-}^{-}$is increased because the hyperbolic passage is on the back side of the planet and $V_{\infty}$ is deflected in the counter-clockwise direction by an angle $\delta . V_{s}^{-}$will decrease for front side passage, $V_{\infty}$ will then be rotated in the clockwise direction for the same angle $\delta$, provided $r_{p}$ is the same. For front side passage expressions for $V_{\theta_{s}}$ and $\epsilon$ will be slightly different, i.e.,

$$
V_{\theta_{s}}=V_{p}-V_{\infty}^{+} \cos \epsilon ; \epsilon=\alpha-\delta .
$$

The above relations are valid for both conventional gravity assist and aero-gravity assist, except that $\delta$ is different for the two cases and $V_{\infty}^{+}$is different from $V_{\infty}^{-}$for AGA.

For the analysis of AGA or GA, several parameters of the overall flight profile must be specified. These include the initial Earth orbit radius at launch, type of heliocentric transfer, distance of closest approach to the planet, and front side or back side passage.

We have assumed in our analysis that the heliocentric transfer trajectory is a single impulse transfer and that the heliocentric orbits of the planets are circular and coplanar.

As mentioned earlier, the angle $\delta$ appearing in the expression of $\epsilon$ in Eq. (2.18) and Eq. (2.21) for front side and back side passage respectively, would be different for AGA and GA. $\delta$ for GA is given in Eq. (2.19), but that for specific AGA-must be calculated. Before doing that, we will discuss the co-ordinate system and equations of motion of the spacecraft during its flight in the planetary atmosphere.

\subsection{Equations of Motion}

With respect to the planet-fixed system $O X Y Z$ (Fig. 2.2), the position vector $\mathbf{r}$ is defined by the magnitude $r$, its longitude $\theta$ (measured from the $X$-axis, in the equatorial plane, positive in the counter-clockwise direction) and its latitude $\phi$ (measured from the equatorial plane, along a meridian, positive towards the North Pole). $\gamma$ is 


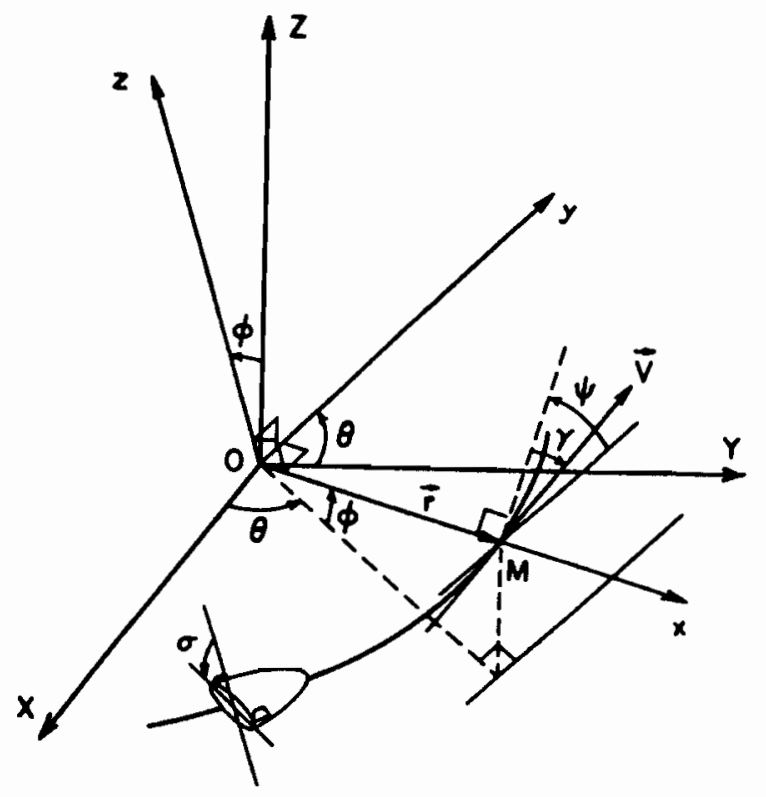

Figure 2.2: Co-ordinate system.

the flight path angle, the angle between the velocity vector $\mathrm{V}$ and the local horizontal plane, that is the plane passing through the vehicle located at point $M$ and orthogonal to the position vector $\mathbf{r}$. It is positive when $\mathbf{V}$ is above the horizontal plane. $\psi$ is the heading angle, i.e., the angle between the local parallel of the latitude and the projection of $\mathbf{V}$ on the horizontal plane. It is positive in the right-handed direction about the $x$-axis. $\mathbf{i}, \mathbf{j}$, and $\mathbf{k}$ are the unit vectors along the axes of the rotating system Oxyz. Next the equations governing the motion of the spacecraft in a planetary atmosphere are given.

\subsubsection{Equations Governing Flight over a Rotating Spherical Planet}

The general three dimensional motion can be described either by three second order differential equations (involving second order derivatives of $r, \theta, \phi$ ) or by an equivalent set of six first order equations (involving first order derivatives of $r, \theta, \phi, V, \gamma, \psi$ ). The usage of the second approach is more common in atmospheric flight mechanics.

The equations governing the atmospheric trajectory are obtained by assuming the spacecraft to be a point mass and then using Newton's second law. However, to calculate the aerodynamic forces, the spacecraft must be treated as a finite body. 
The determination of the aerodynamic forces will be discussed in a later section.

The atmospheric flight of the spacecraft will be analyzed with the following assumptions. The vehicle has a lifting configuration and the lift can be modulated by varying the angle of attack. Lift modulation is the sole means of controlling the flight path angle in the atmosphere. Furthermore, the atmospheric properties, the vehicle's aerodynamic properties and other parameters appearing in the equations of motion, as well as the initial position and velocity of the vehicle, are all known precisely. The effect of the uncertainty in the atmospheric density will be considered in Chapter 5 .

The equations of motion relative to a rotating spherical planet are (Vinh et al. 1980, Vinh 1993)

$$
\begin{aligned}
\frac{d r}{d t}= & V \sin \gamma, \\
\frac{d \theta}{d t}= & \frac{V \cos \gamma \cos \psi}{r \cos \phi}, \\
\frac{d \phi}{d t}= & \frac{V \cos \gamma \sin \psi}{r}, \\
\frac{d V}{d t}= & \frac{F_{T}}{m}-g_{p} \sin \gamma+\omega^{2} r \cos \phi(\sin \gamma \cos \phi-\cos \gamma \sin \psi \sin \phi), \\
V \frac{d \gamma}{d t}= & \frac{F_{N} \cos \sigma}{m}-g_{p} \cos \gamma+\frac{V^{2} \cos \gamma}{r}+2 \omega V \cos \psi \cos \phi \\
& +\omega^{2} r \cos \phi(\cos \gamma \cos \phi+\sin \gamma \sin \psi \sin \phi), \\
V \frac{d \psi}{d t}= & \frac{F_{N} \sin \sigma}{m \cos \gamma}-\frac{V^{2} \cos \gamma \cos \psi \tan \phi}{r}+2 \omega V(\tan \gamma \sin \psi \cos \phi-\sin \phi) \\
& -\frac{\omega^{2} r}{\cos \gamma} \cos \psi \sin \phi \cos \phi,
\end{aligned}
$$

where $t=$ time; $F_{T}=T_{p} \cos \alpha_{t}-D ; F_{N}=T_{p} \sin \alpha_{t}+L ; D=$ aerodynamic drag force; $L=$ aerodynamic lift force; $T_{p}=$ propulsive thrust; $\alpha_{t}=$ angle between the velocity and thrust vectors (see Fig. 2.3); $m=$ mass of the spacecraft; $\omega=$ angular velocity of rotation of the planet.

We have assumed that the value of $g_{p}$ is based on the Newtonian gravitational field, therefore $g_{p}=\mu / r^{2}$, where $\mu$ is the universal gravitational constant $G$ multiplied by the mass of the planet (Note that $g_{p}$ is not a constant).

Equations (2.22-2.24) can be obtained from kinematics while Eqs.(2.25-2.27) are the force balance equations. If we assume that the atmosphere is at rest with respect 
to the planet, then it has the same rotation as the planet. For the planets which will be considered here for AGA, $\omega$ is very small and the term $\omega^{2} r$, which is the transport acceleration, will also be small and can be neglected. On the other hand, the term $2 \omega V$, which represents the Coriolis acceleration, might have an important effect in a high speed, long range flight. For an accurate analysis, especially in the problem of computing the trajectory of a ballistic missile, this term should be retained. However, our analysis will be restricted to two planets, Venus and Mars. For Venus, the Coriolis acceleration will be negligible because $\omega$ for Venus is approximately 240 times smaller than that of the Earth. In the case of Mars, $g_{p}$ is small (approximately 1/2.6 times that of the Earth) and the main forces involved in the calculations are the centrifugal effect $\left(V^{2} / r\right)$, and aerodynamic lift and drag, while the Coriolis acceleration will be around half of $g_{p} \ll V^{2} / r$. Hence we would neglect it and assume that the planet is non-rotating.

\subsubsection{Equations Governing Flight over a Non-Rotating Planet}

If $\omega$ is put equal to zero in Eqs.(2.24-2.26), one obtains

$$
\begin{aligned}
\frac{d r}{d t} & =V \sin \gamma, \\
\frac{d \theta}{d t} & =\frac{V \cos \gamma \cos \psi}{r \cos \phi}, \\
\frac{d \phi}{d t} & =\frac{V \cos \gamma \sin \psi}{r}, \\
\frac{d V}{d t} & =\frac{F_{T}}{m}-g_{p} \sin \gamma \\
V \frac{d \gamma}{d t} & =\frac{F_{N} \cos \sigma}{m}-g_{p} \cos \gamma+\frac{V^{2} \cos \gamma}{r} \\
V \frac{d \psi}{d t} & =\frac{F_{N} \sin \sigma}{m \cos \gamma}-\frac{V^{2} \cos \gamma \cos \psi \tan \phi}{r} .
\end{aligned}
$$

Since thrust will not be used in the maneuvers considered in this thesis, the term $T_{p}$ is removed from the above equations of motion and hence $F_{T}=-D$ and $F_{N}=L$. Equations (2.28-2.33) will be used in our analysis instead of Eqs.(2.22-2.27).

The energy needed to change the flight path angle or bank angle by control surfaces is negligible compared to the energy of the spacecraft; therefore we have not included it in our calculations. 


\subsection{Aerodynamic Forces}

Besides the gravitational force of attraction, the lifting vehicle flying inside the atmosphere of a planet is also subjected to aerodynamic forces. The discussion below contains the basic elements of how to calculate the aerodynamic force necessary for the analysis of the optimal trajectories in atmospheric flight.

\subsubsection{The Atmosphere}

To analyze the effects of the aerodynamic forces acting on a vehicle in flight, it is necessary to model the planetary atmosphere in which the flights take place. Our analysis will be restricted to skip trajectories i.e., trajectories in which a vehicle enters the upper atmosphere, maneuvers to satisfy its objectives and then leaves the atmosphere for space. The more complicated aspects of planetary atmosphere are of no consequence in our aerodynamic calculations. For instance, though the atmosphere is composed of a mixture of a number of gases, it may be treated as a uniform gas of unvarying composition throughout the aerodynamically significant altitudes. The particular composition of the atmosphere can have an important influence only on the aerodynamic heating of the vehicle because of the details of dissociation of the gases after passing through the vehicle's bow shock wave. For performance analysis, the concern in modelling the atmosphere will be to conveniently and accurately represent the density variation with the altitude.

The physical properties of interest for planetary atmosphere are temperature, pressure, density and viscosity. It is a fact that the values of these properties vary, not only with geographic location and altitude, but also from day to day. In flight mechanics calculation, we adopt a specific set of data representing some kind of average conditions. Here we will only discuss the density because temperature, viscosity and pressure are not explicitly related to the aerodynamic forces, but affect the motion through density (Vinh 1981).

It will be assumed here that the atmospheric density $\rho$ increases exponentially 
with the decrease of altitude, that is,

$$
\rho=\rho_{0} e^{-\left(r-r_{r}\right) / S_{h}}
$$

where $\rho_{0}$ is the density at the reference altitude $\left(r_{r}-r_{0}\right), r_{0}$ is radius of the planet and $S_{h}$ is a scale height. The value of $S_{h}$ can be found by fitting the observed atmospheric data of the planet to the exponential relation given in Eq.(2.34).

\subsubsection{Drag and Lift Forces}

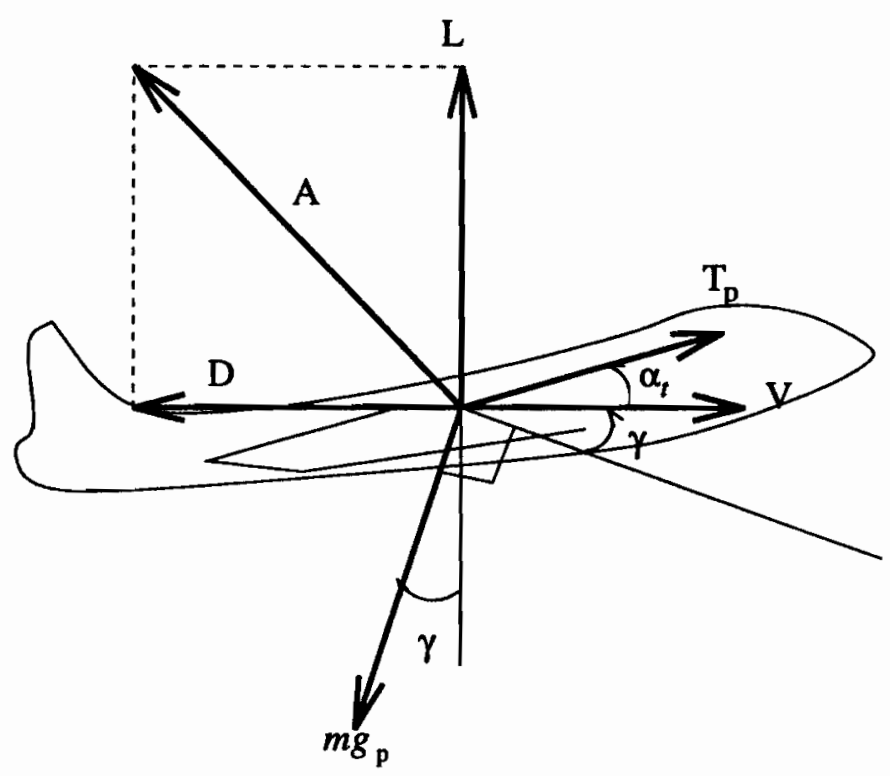

Figure 2.3: Aerodynamic forces in the plane of symmetry.

Aerodynamic forces develop whenever there exists a relative motion between the fluid and the immersed body. The aerodynamic force on a body depends on its shape and its attitude, that is its relative orientation with respect to the flow of the fluid. For a given shape and attitude, aerodynamic force $A$ depends on the density, the free stream velocity, the size of the body, the viscosity and the speed of sound in the fluid. Thus we can write

$$
A=C_{A} \rho S V^{2}
$$


where $C_{A}$ is a certain dimensionless coefficient which is a function of the shape of the body, its attitude with respect to the relative velocity $V$ of the fluid, velocity of sound and viscosity. The size of the body can be expressed through the reference area $S$. The attitude of the vehicle can be described conveniently by the angle of attack $\alpha_{a}$, which is the angle between the relative velocity vector and a reference line fixed with respect to the vehicle. In the equations of motion, it is convenient to divide the aerodynamic forces into two forces: the drag force opposite to the direction of the motion, and the lift force orthogonal to it. Hence we have

$$
\begin{aligned}
D & =\frac{1}{2} \rho S V^{2} C_{D}, \\
L & =\frac{1}{2} \rho S V^{2} C_{L},
\end{aligned}
$$

where $C_{D}$ and $C_{L}$ are called the drag and lift coefficient, respectively. These are functions of the angle of attack $\alpha_{a}$, Reynolds number $R_{e}=V l / \nu$ ( $l$ is a reference length of the vehicle and $\nu$ is the kinematic viscosity) and Mach number $M$. Therefore we have

$$
\begin{aligned}
C_{D} & =C_{D}\left(\alpha_{a}, M, R_{e}\right), \\
C_{L} & =C_{L}\left(\alpha_{a}, M, R_{e}\right) .
\end{aligned}
$$

\subsubsection{The Drag Polar}

At given $M$ and $R_{e}$, the coefficients $C_{D}$ and $C_{L}$ are function of $\alpha_{a}$. By eliminating $\alpha_{a}$ from Eqs.(2.38) and (2.39) one obtains

$$
C_{D}=C_{D}\left(C_{L}, M, R_{e}\right)
$$

The aerodynamic configurations of most vehicles can be expressed by the generalized drag polar:

$$
C_{D}=C_{D_{o}}\left(M, R_{e}\right)+K\left(M, R_{e}\right) C_{L}^{n},
$$

where $C_{D_{o}}$ (zero lift drag coefficient) denotes the drag coefficient at zero $C_{L}$, i.e. zero $\alpha_{a}$, while $K C_{L}{ }^{n}$ is called the induced drag. 
For constant $M$ and $R_{e}$, the coefficients $C_{D_{o}}$ and $K$ are constant and can be selected to best represent the drag polar obtained through wind tunnel measurements. For subsonic flight, $n=2$ can be used and the drag polar is called the parabolic polar. The wind tunnel experiments also show that the parabolic polar is reasonably valid for hypersonic regime up to $M=8$ (Penlund et al. 1983). However, for a threedimensional body at very high Mach number $(M>20)$ when the ratio of specific heats $c_{p} / c_{v}$ becomes close to 1 due to ionization caused by high temperature, results closer to the real situation can be obtained by using $n=3 / 2$ in the drag polar which corresponds to the Newtonian theory valid for hypersonic regime (Miele 1966, Anderson 1989, Shevell 1989). Previous investigators have considered $n=2$ for maneuvering through the atmosphere for "aero-assisted orbit transfer" and "reentry flight mechanics" even though in all cases the Mach numbers of the vehicles were very high $(M>20)$. The reason is probably the simplicity of the parabolic polar model. In our analysis, we will consider both values of $n$ for the hypersonic regime, mainly because the velocity range of our hypersonic flight will be very high $(M>50)$, which will make $n=2$ inappropriate, but also for comparison purposes.

The $L / D$ ratio can be written as

$$
E=L / D=\frac{C_{L}}{C_{D_{o}}+K C_{L}^{n}} .
$$

Clearly, $E$ reaches its maximum when $d E / d C_{L}=0$, that is when

$$
C_{L}=C_{L}^{*}=\left(\frac{C_{D_{\circ}}}{(n-1) K}\right)^{\frac{1}{n}} .
$$

The corresponding value of $E$ is

$$
E^{*}=\frac{C_{L}^{*}}{C_{D}^{*}}=\frac{1}{n}\left(\frac{1}{K}\right)^{\frac{1}{n}}\left(\frac{n-1}{C_{D_{\mathrm{o}}}}\right)^{\frac{n-1}{n}},
$$

where $E^{*}$ is the maximum value of $L / D$, while $C_{L}^{*}$ and $C_{D}^{*}$ are the corresponding lift and drag coefficients, respectively. To simplify the analysis, we assume that the liftto-drag relationship is independent of the Mach number and Reynolds number. This assumption is essentially correct for flight in the hypersonic regime. We introduce a rescaled lift coefficient $\lambda$ defined by

$$
\lambda=\frac{C_{L}}{C_{L}^{*}} .
$$


When $C_{L}$ reaches its maximum, $\lambda$ has its maximum value, $\lambda_{\max }$. We can now rewrite the $L / D$ relationship for constant $M$ and $R_{e}$ as

$$
C_{D}=C_{D}^{*} f(\lambda), \text { or } C_{D}=\frac{C_{L}^{*} f(\lambda)}{E^{*}}
$$

where

$$
f(\lambda)=\frac{(n-1)+\lambda^{n}}{n} .
$$

Now the above relation can be substituted in the equations of motion Eqs.(2.28-2.33).

\subsection{Heliocentric Velocity after AGA}

By controlling the atmospheric trajectory component we can change the hyperbolic trajectory component after AGA, and eventually $V_{s}^{+}$can be increased or decreased. In this section, we will derive an expression for $V_{s}^{+}$after AGA. The cases of planar and three-dimensional atmospheric flight will be dealt separately and in each case the cumulative deflection angle of all three components of AGA trajectory is incorporated into the calculation of $V_{s}^{+}$. Later, an optimization technique will be applied to the atmospheric component to obtain the extremum $V_{s}^{+}$(maximum for outer planet missions and minimum for solar missions).

\subsection{1 $\quad V_{s}^{+}$after Planar Atmospheric Flight}

We have mentioned above in Section 2.3 that the expression for heliocentric velocity of the spacecraft $V_{s}^{+}$after aero-gravity assist or conventional gravity assist is given by the same Eqs.(2.17)-(2.18) except that the term $\epsilon$ is different. For AGA, when augmenting $V_{s}^{+}, \epsilon$ after planar atmospheric flight is given by

$$
\epsilon=\pi-\alpha-\delta
$$

where $\delta$ can determined from Fig. 2.4 as

$$
\delta=\frac{\delta_{1}}{2}+\frac{\delta_{2}}{2}+\theta_{f}-\left|\theta_{1}\right|-\theta_{2} .
$$




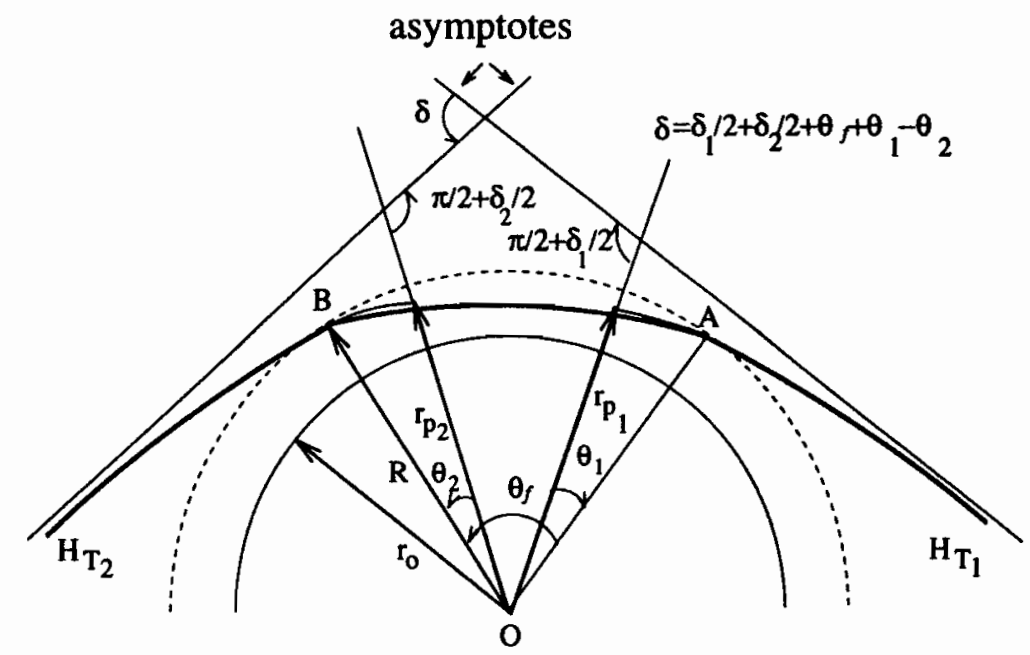

Figure 2.4: Geometry of aero-gravity assist trajectory.

Using the properties of a hyperbolic trajectory, one can write (Kaplan 1976)

$$
\begin{aligned}
\theta_{i} & =\tan ^{-1}\left(\frac{R V_{i}^{2} \sin \gamma_{i} \cos \gamma_{i}}{R V_{i}^{2} \cos ^{2} \gamma_{i}-\mu}\right), \\
\delta_{i} & =2 \sin ^{-1}\left(\frac{1}{e_{i}}\right), \\
e_{i} & =\left[\left(R V_{i}^{2} / \mu-1\right)^{2} \cos ^{2} \gamma_{i}+\sin ^{2} \gamma_{i}\right]^{1 / 2},
\end{aligned}
$$

where $i=1$ and 2 , and the subscript $f$ stands for quantities at the completion of the atmospheric maneuvering at point B in Fig. 2.4. Subscripts $i=1$ and 2 denote quantities for the two hyperbolic planetocentric trajectories $H_{T_{1}}$ and $H_{T_{2}}$ passing through $\mathrm{A}$ and $\mathrm{B}$, respectively. $\theta_{1}$ and $\theta_{2}$ are the angles between their hypothetical closest approach radial lines, $r_{p_{1}}$ and $r_{p_{2}}$, and lines $\mathrm{AO}$ and $\mathrm{BO}$, respectively as shown in Fig. 2.4. In previous studies (McRonald and Randolph 1990, Anderson et al. 1990) $\theta_{1}$ and $\theta_{2}$ were not taken into account. But for more accurate results, these quantities should be included in the calculations. Furthermore, using Eq.(2.15) we have

$$
V_{1}=\left[\left(V_{\infty}^{-}\right)^{2}+2\left(\frac{\mu}{R}\right)\right]^{1 / 2}, \quad V_{\infty}^{+}=\left[V_{f}^{2}-2\left(\frac{\mu}{R}\right)\right]^{1 / 2}
$$

where $R$ is the radius of the sensible atmosphere, $V_{1}$ is the planetocentric velocity at point $\mathrm{A}$ in Fig. 2.4, and $V_{2}=V_{f}$, the planetocentric velocity at point $\mathrm{B}$ in Fig. 2.4. By substituting Eqs. (2.49)-(2.51) in Eqs. (2.47)-(2.48) and then substituting Eq. (2.47) in Eqs. (2.17) and (2.18) we will get the value of $V_{s}^{+}\left[V_{\infty}^{+}\right.$and $V_{1}$ will be calculated by using Eqs. (2.52)]. 


\subsection{2 $V_{s}^{+}$after 3-Dimensional Atmospheric Flight}

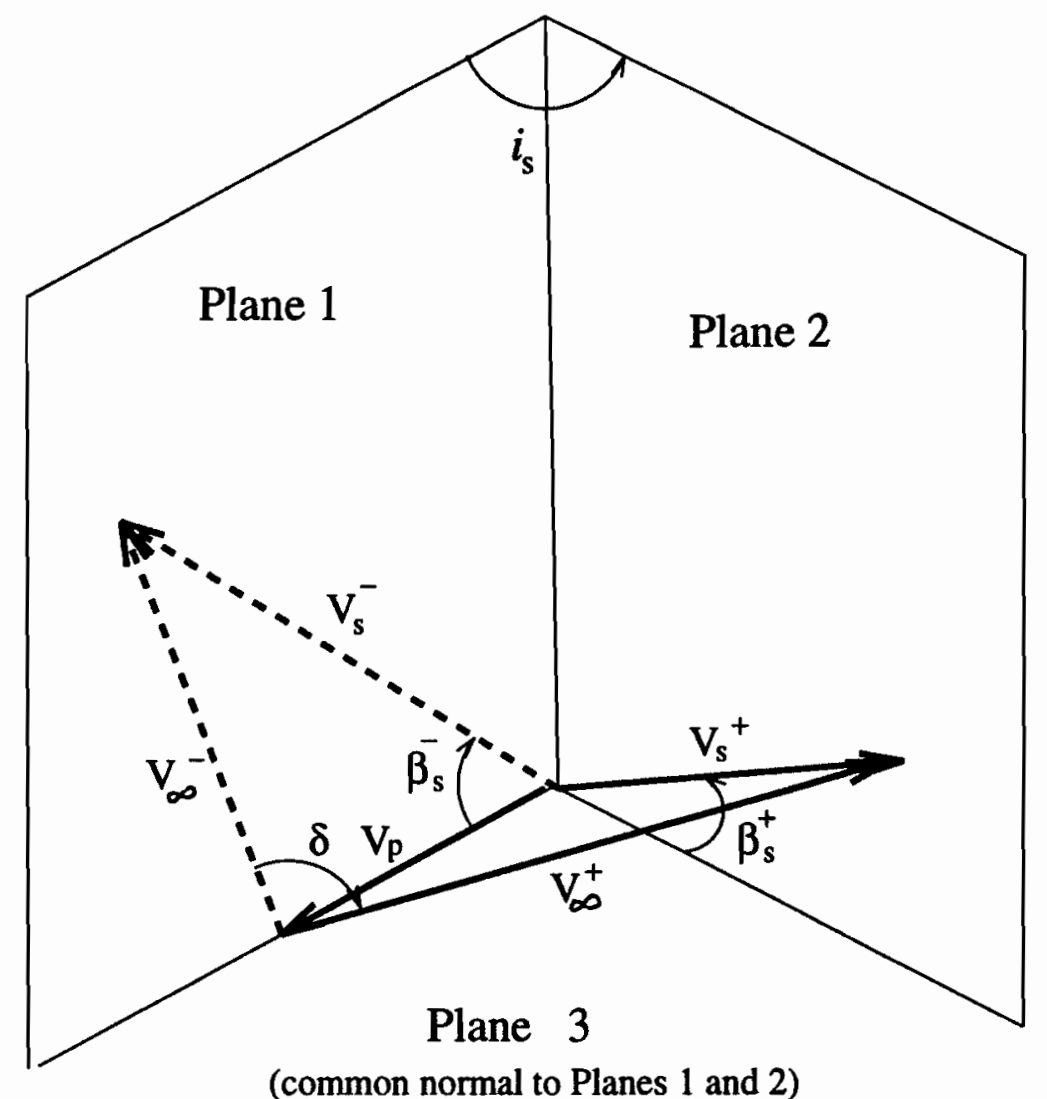

Figure 2.5: Velocity vectors, planes, and angles involved in aero-gravity assist with heliocentric plane change $i_{s}$.

In the case of AGA involving 3 -dimensional atmospheric flight, $V_{s}^{+}$will be in a different plane (Plane 2 in Fig. 2.5) from the one in which $V_{\infty}^{-}$and $V_{s}^{-}$lie (Plane 1 in Fig. 2.5). Here we assume that the heliocentric orbital plane of the planet and that of the spacecraft are the same prior to the atmospheric flight. After maneuvering through the atmosphere the plane of the spacecraft trajectory attains an inclination of $i_{s}$ with respect to the orbital plane of the planet (see Fig. 2.5)

Figure 2.6 shows the geometry of the atmospheric flight and the associated variables $\theta, \phi$ and $\psi$. The plane before the atmospheric flight is the reference plane $O M_{o} N . M_{o}$ is the point where the spacecraft enters the atmosphere of the planet and is in the reference plane. $M$ is the position of the vehicle at time $t_{f}$ (i.e., after completion of the atmospheric flight) in the osculating plane $O I M$. 


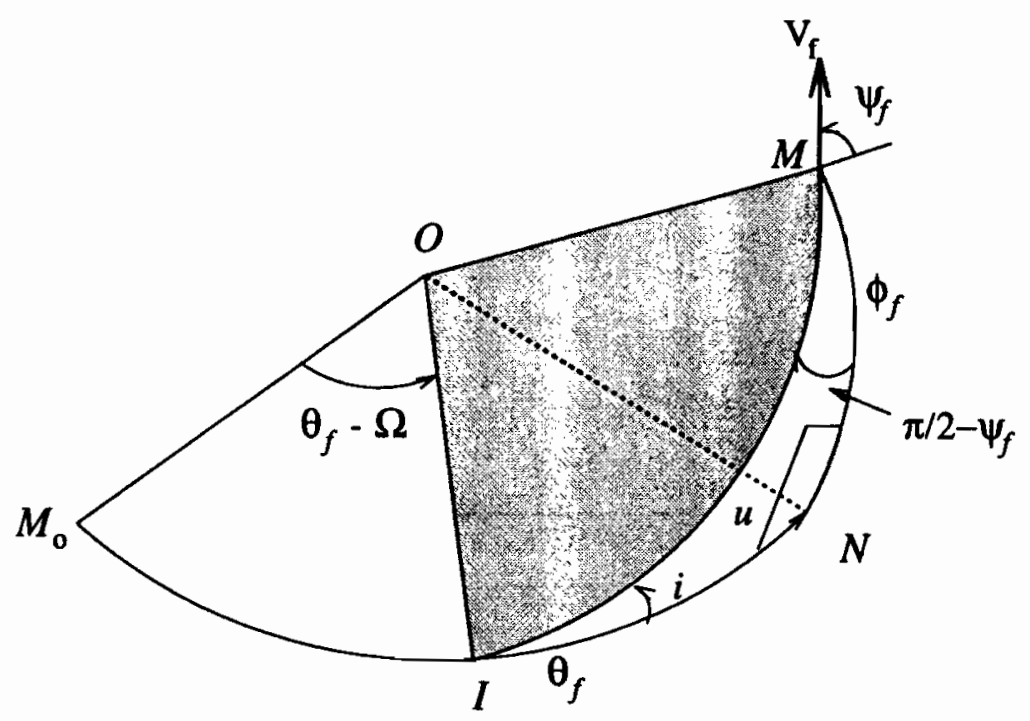

Figure 2.6: Geometry of trajectory variables and planetocentric orbital elements with respect to the initial plane $O M N$.

By using spherical trigonometry we get the planetocentric plane change $i$ at the completion of the atmospheric flight by considering the right spherical triangle INM as

$$
i=\cos ^{-1}\left(\cos \phi_{f} \cos \psi_{f}\right) .
$$

To find $\epsilon$ after atmospheric flight with planetocentric plane change $i$, we have to find expression for the angle between the velocity of the planet $V_{p}$ and $V_{\infty}^{+}$(see Fig. 2.1). Now in Fig. 2.7, the projection of $V_{\infty}^{+}$in the plane $O M_{0}^{\prime} N^{\prime}$ will subtend an angle $\delta^{\prime}$ with $\boldsymbol{V}_{\infty}^{-}$. Therefore, we have

$$
\delta=\cos ^{-1}\left(\cos \phi_{t} \cos \delta^{\prime}\right)
$$

where

$$
\begin{gathered}
\delta^{\prime}=\frac{\delta_{1}}{2}+\theta_{f}+\theta_{1}+\tan ^{-1}(\cos i \tan \Lambda)-\Omega, \\
\Lambda=u+\frac{\delta_{2}}{2}-\theta_{2},
\end{gathered}
$$

and $\phi_{t}$ is the latitude angle at a far away distance from the planet (subscript $t$ shows quantities at far away distance from the planet) and is given by

$$
\phi_{t}=\sin ^{-1}\{\sin i \sin (\pi / 2+\Lambda)\} \text {. }
$$


From Fig. 2.6, $\Omega$ is the angle between $O I$ and $O N$, given by

$$
\Omega=\tan ^{-1}(\tan u \cos i) \text { or } \Omega=\sin ^{-1}\left(\tan \phi_{f} / \tan i\right)
$$

where $u$ is the angle between $O I$ and $O M$ and is given by

$$
u=\sin ^{-1}\left(\frac{\sin \phi_{f}}{\sin i}\right) \text { or } u=\cos ^{-1}\left(\cos \Omega \cos \phi_{f}\right) .
$$

In Fig. 2.7, the vectors shown by dashed lines lie on the $O M_{o}^{\prime} N^{\prime}$ plane, while the vectors shown by solid lines lie in the $O M_{o}^{\prime} M^{\prime}$ plane. From Fig. 2.7, when augmenting $V_{s}^{+}$, we get

$$
\epsilon=\pi-\alpha_{t}
$$

where $\alpha_{t}$ is the angle between $V_{p}$ and $V_{\infty}^{+}$, the one needed to determine $\epsilon$, and is defined as

$$
\alpha_{t}=\cos ^{-1}\left(\cos \theta_{t} \cos \phi_{t}\right)
$$

and $\theta_{t}=\delta^{\prime}+\alpha$. For front side passage to reduce $V_{s}^{-}, \epsilon=\alpha_{t}$ and $\theta_{t}=\alpha-\delta^{\prime}$.

We proceed to derive an expression for the heliocentric plane change $i_{s}$. From Fig. $2.5, \zeta$ is the angle between $V_{p}$ and $V_{s}^{+}$, and can be written as

$$
\zeta=\tan ^{-1}\left(V_{r_{s}} / V_{\theta_{s}}\right)
$$

where $V_{r s}$ and $V_{\theta_{\theta}}$ are defined in Eqs. (2.18).

Figure 2.8(a) shows that $\phi_{t}^{\prime}$ is the angle between $V_{s}^{+}$and its projection on $O_{1} N_{1} P$ plane, and is given by

$$
\phi_{t}^{\prime}=\sin ^{-1}\left(\frac{V_{\infty}^{+} \sin \phi_{t}}{V_{s}^{+}}\right) \text {, }
$$

and

$$
i_{t}^{\prime}=\sin ^{-1}\left(\frac{\sin \phi_{t}^{\prime}}{\sin \zeta}\right)
$$

Now we can derive an expression for $i_{s}$, the heliocentric plane change angle after aero-gravity assist, from Fig. 2.8(b) as

$$
i_{s}=\tan ^{-1}\left(\tan \zeta \cos i_{n}\right)
$$




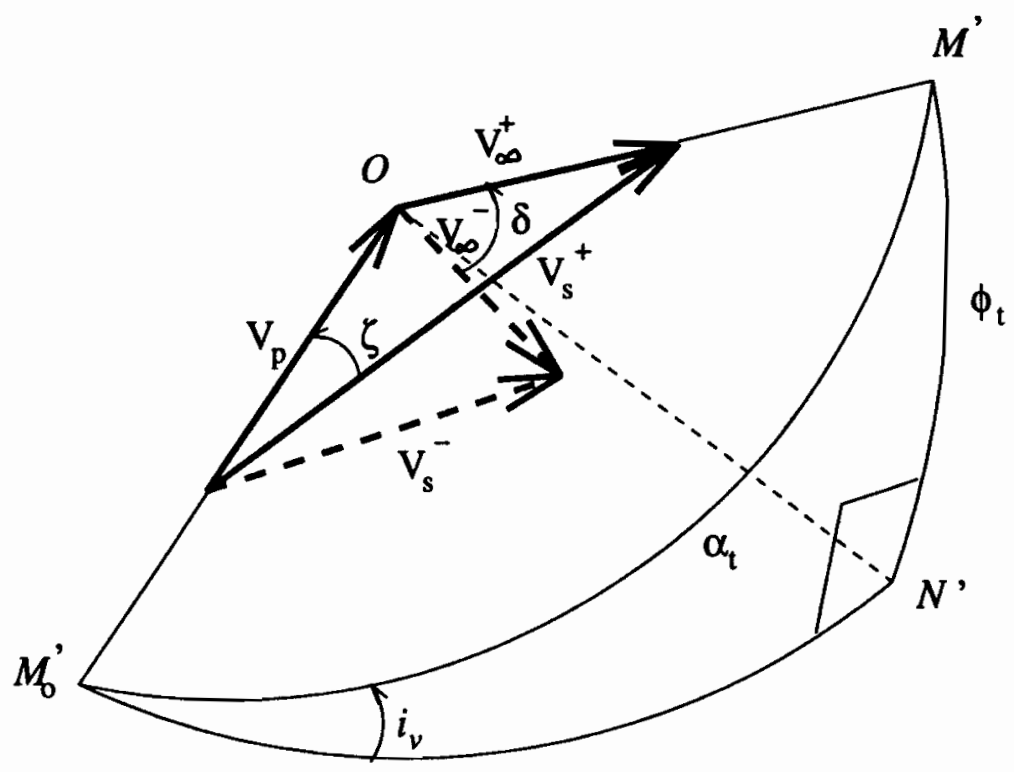

Figure 2.7: Right spherical triangle showing velocity vectors and angles after aerogravity assist.

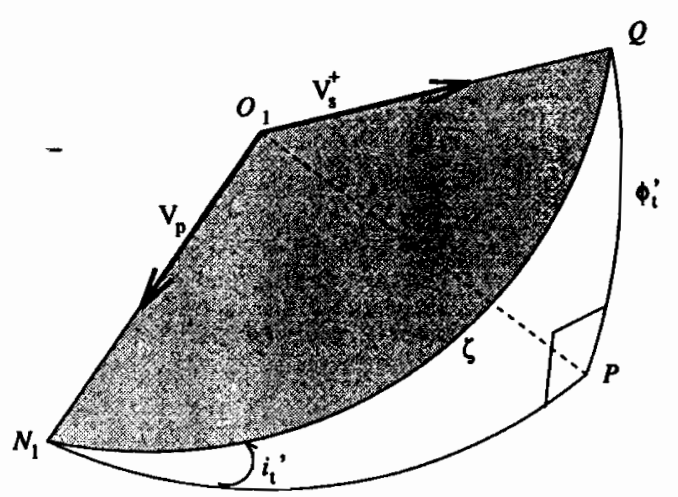

(a)

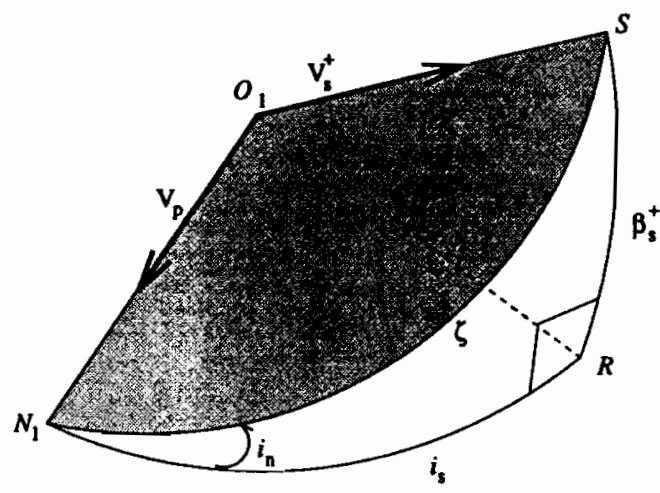

(b)

Figure 2.8: Right spherical triangles showing heliocentric plane change $i_{s}$, heliocentric flight path angle $\beta_{s}^{+}$, and other angles. 
where $i_{n}=\pi / 2-i_{t}{ }_{t}$, because the plane $O_{1} N_{1} P$ (identical to Plane 1 in Fig. 2.5) is perpendicular to plane $O_{1} N_{1} R$ in Fig. 2.8(b) (Plane 3 in Fig. 2.5). Thus we have a new heliocentric flight path angle

$$
\beta_{s}^{+}=\tan ^{-1}\left(\tan i_{n} \sin i_{s}\right)
$$

Now let us consider the case when initially the spacecraft trajectory has a heliocentric inclination $i_{s_{o}}$ with respect to the orbital plane of the planet prior to AGA. This case is similar to the one shown in Fig. 2.8(b); if we know $\beta_{s}^{-}$then we can find $i_{n}$ by using an equation similar to Eq. (2.66) after replacing $i_{s}$ with $i_{s_{o}}$ and $\beta_{s}^{+}$with $\beta_{s}^{-}$as

$$
i_{n}^{\prime}=\tan ^{-1}\left(\frac{\tan \beta_{s}^{-}}{\sin i_{s_{o}}}\right)
$$

The heliocentric inclination angle $i_{s}$ after atmospheric maneuvering for this case is given by

$$
i_{s}=\tan ^{-1}\left(\tan \zeta \cos i_{n}\right),
$$

where $i_{n}=i_{n}^{\prime}-i_{t}^{\prime}$ and we have

$$
\beta_{s}^{+}=\tan ^{-1}\left(\tan i_{n} \sin i_{s}\right)
$$

\subsection{Aerodynamic Heating Rate}

During the flight of the spacecraft in the planetary atmosphere its kinetic energy decreases. A part of this energy is converted into heat absorbed by the vehicle. Here it will be assumed that only the maximum temperature in the stagnation region of the vehicle is of concern. To control this temperature, it suffices to control the heating rate in that region. Whether AGA is feasible or not, will depend on the level of heating rate in a given scenario.

The aerodynamic convective heating rate, $q_{c}$, along the atmospheric trajectory is computed according to the equation (Anderson 1989, Vinh et al. 1980)

$$
q_{c}=\frac{k \rho^{1 / 2} V^{3}}{\left(r_{n}\right)^{1 / 2}}
$$


where $r_{n}$ is the nose radius of the stagnation region, while $k$ is a constant dependent on the constituent gases of the atmosphere. We have already indicated above that the composition of the atmospheric gases is important for calculating the aerodynamic heating rate. In the numerical calculations, AGA of Venus and Mars has been considered, whose atmospheres are assumed here to contain $85 \% \mathrm{CO}_{2}$ and $15 \% \mathrm{~N}_{2}$ (in reality, the atmospheres of Venus and Mars contain respectively $90 \% \mathrm{CO}_{2}, 10 \% \mathrm{~N}_{2}$, and $95 \% \mathrm{CO}_{2}, 5 \% \mathrm{~N}_{2}$, but the values of $k$ for $90 / 10$ and $95 / 5$ mixtures are not available in the literature; in any case, $k$ is not very sensitive to the mixture ratio). The value of $k$ for the $85 / 15$ mixture is given by Sutton and Graves (1971), which is $1.8425 \times 10^{-8}$ (and is not likely to be very different from the real values; note that even for $50 / 50$ mixture, $k=1.7765 \times 10^{-8}$ and is only $5 \%$ lower). The above value of $k$ assumes that $q_{c}$ is in Watt $/ \mathrm{cm}^{2}, \rho$ in $\mathrm{kg} / \mathrm{m}^{3}, V$ in m/s and $r_{n}$ in $\mathrm{m}$.

The radiative heating rate is computed using (Tauber and Sutton 1991)

$$
q_{r}=F r_{n}^{a} \rho^{p} f(V)
$$

where $f(V)$ are tabulated values that are dependent on the flight velocity $V$ and the atmospheric composition. The exponents $a$ and $p$ are functions of $\rho$ and $V$. According to Page and Woodward (1972), at high velocities (e.g. $10 \mathrm{~km} / \mathrm{s}$ ) the radiative heating rate is more or less the same for the atmospheres of the Earth, Venus, and Mars. The velocities of spacecraft in the present case will be higher than the above velocity. Actually, at a very high Mach number the atmospheric gases dissociate, become a partial ionized plasma and the radiative heating rate for the Earth, Venus and Mars become approximately equal. Therefore the following values, which are for air, can be used:

$$
F=4.736 \times 10^{4} ; a=1.072 \times 10^{6} V^{-1.88} \rho^{-0.325} ; p=1.22 .
$$

In Eqs.(2.71) and (2.72), again the heating rate is in Watt $/ \mathrm{cm}^{2}, \rho$ is in $\mathrm{kg} / \mathrm{m}^{3}, V$ is in $\mathrm{m} / \mathrm{s}$ and $r_{n}$ is in $\mathrm{m}$. The nose radius $r_{n}$ has been taken as either 0.5 or $1 \mathrm{~m}$ (in 1989 Randolph and McRonald have used $r_{n}=1 \mathrm{~m}$ and in the studies on AOT mission $r_{n}$ was taken a $1 \mathrm{~m}$ as well). Since only the heating rate range is of concern here, these models will suffice for this thesis. 


\section{Chapter 3}

\section{Optimization Method}

In this chapter we will discuss the optimization method that is used in this thesis to maximize the advantage of AGA. To start with, we will review the basics of optimization theory.

\subsection{Functional Optimization}

Functional optimization solves the problem of finding an unknown function to maximize or minimize a certain functional $J$. Usually, we consider a dynamical system, defined at each instant $t$, by a column vector $x$ with $n$ components $x^{i}(i=1,2 \ldots ., n)$, called the state vector. The system is governed by a system of differential equations called equations of motion or state systems

$$
\dot{\boldsymbol{x}}=f(\boldsymbol{x}, \boldsymbol{u}, t),
$$

where $\boldsymbol{f}$ is a column vector with $n$ components, and $\boldsymbol{u}$ is a column vector with $m$ components $u^{j}(j=1,2, \ldots, m)$ called the control vector. For a space vehicle, assumed to be a point mass, the vector $x$ may represent the set of three components of the position vector, the three components of the velocity vector and the mass, while vector $\boldsymbol{u}$ may represent a set of three components of the thrust, the bank angle and the angle of attack. But here in our analysis no thrust will be applied, therefore the mass component of vector $\boldsymbol{x}$ and thrust component of control vector $\boldsymbol{u}$ will not appear in the equations of motion. 
In the classical calculus of variations, a problem involves of finding an optimal control law $\boldsymbol{u}^{*}(t)$ such that a certain function $J$ of the initial state and time and final state and time

$$
J=J\left(\boldsymbol{x}_{o}, t_{o}, \boldsymbol{x}_{f}, t_{f}\right),
$$

has a stationary value. $J$ is called the performance index. The trajectory is generated from the initial state $\boldsymbol{x}_{o}$ at time $t_{o}$ to the final state $\boldsymbol{x}_{f}$ at time $t_{f}$ by integration of the equations of motion using certain control law $\boldsymbol{u}=\boldsymbol{u}(t)$. Since $J$ depends on the particular vector function $\boldsymbol{u}(t)$ selected, it is a functional. The vector function $\boldsymbol{u}^{*}(t)$ is the unknown function which maximizes or minimizes the functional $J$. For a minimization problem the sign of $J$, performance index for maximization problem, is changed (Vinh 1981).

\subsubsection{Necessary Conditions for Optimality}

Pontryagin's maximum principle states that if the control function $u_{j}$, piecewise continuous on the interval $\left[t_{a}, t_{f}\right]$, is maximizing, then there exists a nontrivial adjoint vector $\boldsymbol{p}$ defined on the same interval. We can form the Hamiltonian $\mathcal{H}$

$$
\mathcal{H}=\boldsymbol{p} \cdot \boldsymbol{f}
$$

so that the adjoint vector $\boldsymbol{p}$ satisfies the differential equation

$$
\dot{p}=-\frac{\partial \mathcal{H}}{\partial \boldsymbol{x}}
$$

If the components of the control vector are subject to the inequality constraint

$$
0 \leq u^{j}<u_{\max }^{j},
$$

with respect to the components of the control vector, the Hamiltonian is maximized when $u^{j}= \pm u_{m a x}^{j}$ or at an interior point. The first order necessary condition for an interior maximum is

$$
\frac{\partial \mathcal{H}}{\partial \boldsymbol{u}}=0 .
$$

The second order optimal condition is 


$$
\frac{\partial^{2} \mathcal{H}}{\partial \boldsymbol{u}^{2}}<0
$$

If time $t$ (or any other independent variable) is not contained explicitly in the Hamiltonian $\mathcal{H}$, then $\partial \mathcal{H} / \partial t=0$ and we have

$$
\mathcal{H}=\text { constant }
$$

Furthermore, if a state variable $x^{i}$ is not contained explicitly in the Hamiltonian $\mathcal{H}$, it is said to be ignorable. The corresponding adjoint equation is

$$
\dot{p^{i}}=-\frac{\partial \mathcal{H}}{\partial x^{i}}=0
$$

i.e., $p^{i}$ is a constant. If $p^{i}$ is not included in the performance index $J$, then

$$
p^{i}=0
$$

If an initial or final state variable is not contained explicitly in the performance index $J$ nor has been specified, then it is called a free parameter; a necessary condition for optimality is that the corresponding adjoint is zero. For example, if $x_{f}^{i}$ is free, then $p_{f}^{i}=0$. This also applies to the independent variable, that is, if $t_{f}$ is free, $\mathcal{H}_{f}=0$. (Note that the Hamiltonian is the adjoint to the independent variable.) Furthermore, if initial or final values of all the state variables are free then the final time must be fixed, otherwise the optimization problem will be meaningless. For example if $x_{f}^{i}$ is free for all $i$, then $p_{f}^{i}=0$; similarly, if $x_{o}^{i}$ is free for all $i$ then $p_{o}^{i}=0$. In either case, the final time (or independent variable) must be specified to solve the optimization problem (Pontryagin et al. 1962; McCausland 1968).

\subsubsection{Transversality Conditions}

In trajectory optimization problems when the beginning or the end point of the state trajectory is free, one needs to introduce a transversality condition to find all the boundary conditions which are required for the solution of the problem. The transversality condition, for the Hamiltonian formulation of the problem under discussion can be shown to be (Vinh 1981; McCausland 1968)

$$
\delta J-\left[-\mathcal{H} \delta t+\boldsymbol{p}_{i} \delta \boldsymbol{x}_{i}\right]_{0}^{f}=0,
$$


where

$$
\delta J=\frac{\partial J}{\partial \boldsymbol{x}_{o}} \delta \boldsymbol{x}_{o}+\frac{\partial J}{\partial t_{o}} \delta t_{o}+\frac{\partial J}{\partial \boldsymbol{x}_{f}} \delta \boldsymbol{x}_{f}+\frac{\partial J}{\partial t_{f}} \delta t_{f}
$$

or explicitly

$$
\begin{aligned}
& \frac{\partial J}{\partial \boldsymbol{x}_{o}}=-\boldsymbol{p}_{o} \\
& \frac{\partial J}{\partial \boldsymbol{x}_{f}}=\boldsymbol{p}_{f} \\
& \frac{\partial J}{\partial t_{o}}=\mathcal{H}_{o} \\
& \frac{\partial J}{\partial t_{f}}=-\mathcal{H}_{f} .
\end{aligned}
$$

\subsection{Method of Solution}

The trajectory optimization problem is a two-point-boundary-value-problem (TPBVP), since it needs boundary conditions to be satisfied at final time $t_{f}$ in addition to some initial conditions while maximizing or minimizing the performance index $J$. In trajectory optimization problems, time is usually free (i.e., it does not appear explicitly in the Hamiltonian and performance index). In this study also, time will be considered free while solving the optimization problem. In those problems in which the time is free, we have $n$ initial conditions. For example if $x_{o}^{i}(i=1,2, . ., n)$ are prescribed, $x_{f}^{i}(i=1,2, \ldots, m)$ are also prescribed, $n-m$ are the transversality conditions, and time is free, then we have to find appropriate $p_{o}^{i}(i=1,2, \ldots, n)$ to satisfy the boundary conditions and to find the final time $t_{f}$ as well. Hence we have $n+1$ final conditions to satisfy for the optimum solution. Therefore this kind of problem has to be transformed into the so-called standard form, which means finding $n+1$ initial conditions to satisfy $n+1$ final conditions at unknown $t_{f}$.

For this purpose we introduce a new state variable $x^{n+1}$ such that (Ascher, Mattheij and Russel 1988; Stoer and Bulirsch 1980)

$$
x^{n+1}=t_{f} \text { and } y=t / t_{f}
$$


and add the ordinary differential equation

$$
\frac{d x^{n+1}}{d y}=0 .
$$

$y$ will be the new independent variable instead of $t$.

We introduce now a new vector $\hat{x}\left(x^{1}, x^{2} \ldots x^{n+1}, p^{1}, p^{2}, \ldots p^{n+1}\right)$ and the system of differential equations in the TPBVP would be:

$$
\frac{d \hat{x}}{d y}=\left[\begin{array}{c}
\dot{x}^{1} \cdot x^{n+1} \\
\dot{x}^{2} \cdot x^{n+1} \\
\vdots \\
\dot{x}^{n} \cdot x^{n+1} \\
0 \\
\dot{p}^{1} \cdot x^{n+1} \\
\dot{p}^{2} \cdot x^{n+1} \\
\vdots \\
\dot{p}^{n} \cdot x^{n+1}
\end{array}\right] .
$$

Thus we have $2 n+1$ ODEs and $2 n+1$ boundary conditions (out of these $n$ boundary conditions to be satisfied at $y=0$ and $n+1$ boundary conditions to be satisfied at $y=1$ ) which is the standard form of a TPBVP. The terminal boundary conditions are applied at $y_{f}=1$. Now the solution can be obtained by using the shooting method. The technique used in the shooting method for solving a TPBVP is explained briefly below.

\subsubsection{Shooting Method}

In the shooting method, we regard the initial unspecified conditions $\hat{x}_{0}^{i}(i=1,2, \ldots, k)$ as variables and seek to determine their values that satisfy the boundary conditions at $y=1$ or $t_{f}$.

The nomenclature "shooting" might have been motivated by the ballistic problem illustrated in Fig. 3.1. Here we have a cannon located at position $x=a$ on the $x$-axis, and a target at $x=b$. If an artillery shell with known initial velocity is subject only 


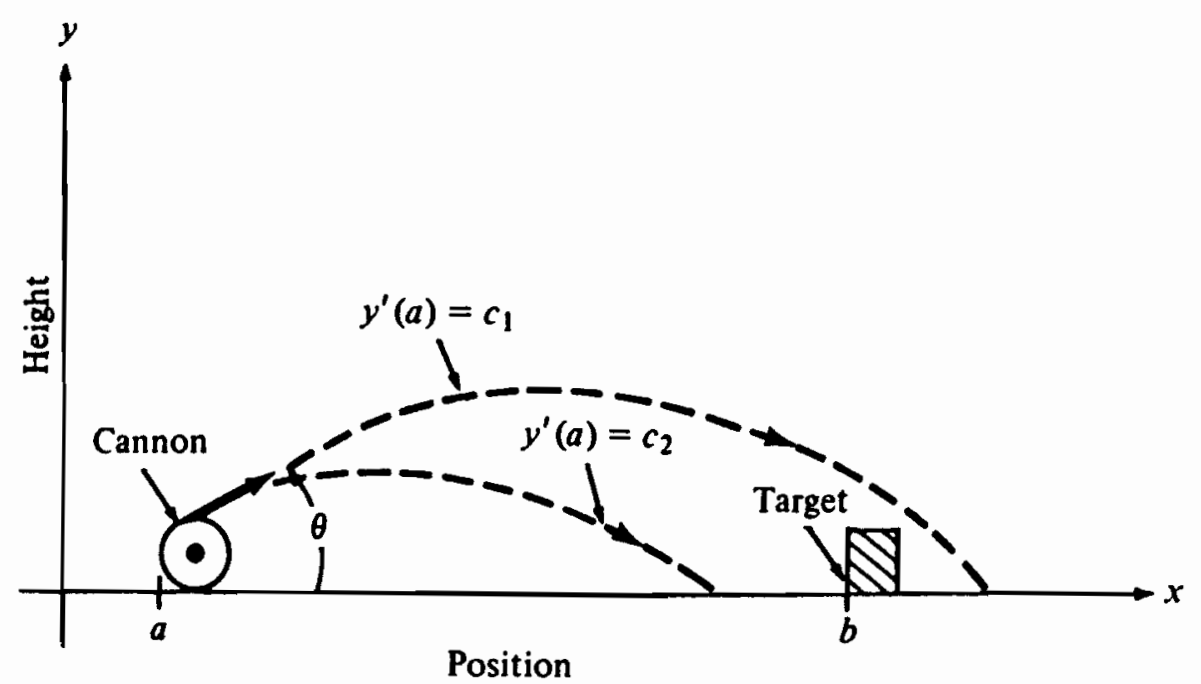

Figure 3.1: Shooting Method application.

to Newton's law of motion, then its trajectory $y(x)$ satisfies a second-order differential equation $y^{\prime \prime}=f\left(x, y, y^{\prime}\right)$. The natural boundary condition is that the height $y(x)$ of the shell be 0 at both $a$ and $b$, or $y(a)=y(b)=0$. Under the shooting method, one adjusts the unknown $y^{\prime}(a)(=\tan \theta)$ or $\theta$ with respect to the error of $y^{\prime}(a)=c_{1}$ or $y^{\prime}(a)=c_{2}$ (see Fig. 3.1) until $y(b)=0$.

In solving the optimization problem of an atmospheric trajectory, the shooting method can be implemented by conjunctive use of a nonlinear algebraic equation solver, such as the Damped Newton Method, for finding required initial conditions, and a differential equation solver, such as Runge-Kutta or Adams-Moulton Method, to calculate the requisite terminal condition needed to satisfy the boundary conditions.

The implementation of the shooting method in solving a TPBVP with nonlinear differential equations and boundary conditions is very difficult and there is no guarantee of convergence. However, the convergence mainly depends upon how good the initial guesses are. Estimates are sometimes available from physical conditions, and sometimes from a graphical representation of solution (Ascher et al. 1988, Stoer and Bulirsh 1980, Yakowitz and Szidarovszky 1986, Conte and de Boor 1980). 


\section{Chapter 4}

\section{Optimal Aero-Gravity Assist}

In this chapter the optimal atmospheric trajectory of the spacecraft for aero-gravity assist is synthesized so as to maximize the heliocentric velocity $V_{s}^{+}$. The problem has been formulated mathematically and necessary conditions for the optimal trajectory and control have been derived using Pontryagin's maximum principle, as discussed in Chapter 3. The results are given for both the drag polars, i.e., when $n=2$ and 1.5 , for the purpose of comparison. The Earth has two neighbouring planets, Mars and Venus, both of which have an atmosphere. Therefore, both Mars-aero-gravity assist and Venus-aero-gravity assist are considered and compared at the end.

\subsection{Equations of Motion}

The equations of motion for planar atmospheric flight, as given in Chapter 2, are

$$
\begin{aligned}
\frac{d r}{d t} & =V \sin \gamma \\
\frac{d \theta}{d t} & =\frac{V \cos \gamma}{r} \\
\frac{d V}{d t} & =-\frac{V^{2} \rho S C_{D}}{2 m}-\frac{\mu}{r^{2}} \sin \gamma, \\
\frac{d \gamma}{d t} & =\frac{V \rho S C_{L}}{2 m}-\left(\frac{\mu}{V r^{2}}-\frac{V}{r}\right) \cos \gamma .
\end{aligned}
$$

When $r>R$, where $R$ is the radius of the sensible atmosphere (see Figs. 1.2 and 2.4), the first terms in Eqs. (4.1c) and (4.1d) disappear and the flight is Keplerian. But for 
$r<R$, the flight is subjected to the atmospheric forces and $C_{L}$ (or $\lambda=C_{L} / C_{L}^{*}$ ) can be used as a control parameter. This corresponds physically to using pitch modulation to shape the trajectory. $C_{L}$ is allowed to assume both positive and negative values. A negative $C_{L}$ value can be interpreted as resulting either from a negative pitch angle or from a positive angle with the vehicle flying upside down.

The independent variable is now changed from $t$ to $\theta$, because that will make it easier to handle the optimization problem. We will then have to solve only three equations instead of four. As shown in Chapter 2, $\theta_{f}$ is included in the performance index. The equations of motion in non-dimensionalized form with $\theta$ as the independent variable are given below for the planar case:

$$
\begin{aligned}
& \frac{d h}{d \theta}=h \tan \gamma, \\
& \frac{d v}{d \theta}=-\frac{A \eta f(n, \lambda) v h}{E^{*} \cos \gamma}-\frac{b \tan \gamma}{h v}, \\
& \frac{d \gamma}{d \theta}=\frac{A h \eta \lambda}{\cos \gamma}+1-\frac{b}{v^{2} h} .
\end{aligned}
$$

The dimensionless variables and parameters used in Eq.(4.2) are defined as follows:

$$
\begin{gathered}
h=\frac{r}{r_{o}} ; v=\frac{V}{\sqrt{\frac{\mu}{R}}} ; b=\frac{R}{r_{o}} ; \\
\eta=\frac{\rho}{\rho_{o}}=e^{-z\left(r-r_{r}\right)} ; z=\frac{1}{S_{h}} ; A=\frac{\rho_{o} S r_{o} C_{L}^{*}}{2 m} .
\end{gathered}
$$

From Eq.(2.46) for $n=2$ and 1.5, $f=\left(1+\lambda^{2}\right) / 2$ and $\left(1+2|\lambda|^{1.5}\right) / 3$, respectively.

\subsection{Optimization Problem}

$V_{s}^{+}$is the heliocentric velocity of the spacecraft after completing the maneuver inside the atmosphere through an angle $\theta_{f}$. We want to maximize $V_{s}^{+}$subject to the differential constraints represented by the equations of motion. Thus we have the performance index

$$
J=V_{s}^{+}
$$


with the constraints represented by Eq.(4.2). Using Eqs.(2.17) and (2.18), we can rewrite $V_{s}^{+}$in dimensionless form

$$
v_{s}^{+}=\left(v_{\theta_{s}}^{2}+v_{r_{s}}^{2}\right)^{1 / 2}
$$

where

$$
\begin{aligned}
v_{\theta_{s}} & =v_{p}+v_{\infty}^{+} \cos \epsilon ; \quad v_{r_{s}}=v_{\infty}^{+} \sin \epsilon ; \\
\epsilon & =\pi-\alpha-\delta ; v_{\infty}^{+}=\left(v_{f}^{2}-2\right)^{1 / 2} ;
\end{aligned}
$$

all the velocities are in dimensionless form (dimensional velocities are divided by $\sqrt{\mu / R})$.

From Chapter 2, we know that

$$
\delta=\frac{\delta_{1}}{2}+\frac{\delta_{2}}{2}+\theta_{f}+\theta_{1}-\theta_{2}
$$

and in terms of dimensionless state variables, we have, for $i=1,2$,

$$
\begin{aligned}
\theta_{i} & =\tan ^{-1} \frac{v_{i}^{2} \sin \gamma_{i} \cos \gamma_{i}}{v_{i}^{2} \cos ^{2} \gamma_{i}-1} \\
\delta_{i} & =2 \sin ^{-1}\left(\frac{1}{e_{i}}\right) \\
e_{i} & =\left[\left(v_{i}^{2}-1\right)^{2} \cos ^{2} \gamma_{i}+\sin ^{2} \gamma_{i}\right]^{1 / 2},
\end{aligned}
$$

where, as mentioned in Chapter 2, the indices 1 and 2 denote quantities for hyperbolic trajectories $H_{T_{1}}$ and $H_{T_{2}}$ at A and B, respectively (see Fig.2.4).

Before deriving optimality conditions, we will briefly explain why we need to optimize the atmospheric trajectory to maximize the advantage of AGA. If we examine Eqs.(4.4-4.6), we can notice that $v_{s}^{+}$is function of $\delta$ and $v_{\infty}^{+}$, while $\delta$ is function of $v_{f}, \gamma_{f}$, and $\theta_{f}$ (subscript $f$ stands for values at the end of atmospheric maneuver). If we increase only $\theta_{f}$, then $v_{f}$ will be reduced too much due to the aerodynamic drag. There will not be much benefit of AGA, in that case. Thus if we want to maximize the advantage of AGA, then we must find the point where $v_{f}$ is not reduced too much due to the aerodynamic drag, at the same time $\delta$ is large enough. Hence to find the maneuver for which $V_{s}^{+}$is maximum we have to use an optimization method. And 
as mentioned above, we will use the Hamiltonian formulation based on Pontryagin's maximum principle for optimizing the atmospheric trajectory associated with AGA.

The spacecraft enters the atmosphere at A (Fig.2.4) and at this point we have

$$
\theta_{e}=0 \quad \text { and } \quad h_{e}=\frac{R}{r_{o}}
$$

and it exits at $B$ with

$$
\theta_{f} \quad \text { unspecified and } h_{f}=\frac{R}{r_{o}}
$$

Now we proceed to derive the necessary conditions for the optimal solution using Pontryagin's maximum principle. From Section 3.1.1, in terms of the adjoint variables $p_{h}, p_{v}$ and $p_{\gamma}$, the Hamiltonian can be formulated for $n=2$ as follows:

$$
\begin{aligned}
\mathcal{H}= & p_{h} h \tan \gamma+p_{v}\left\{\frac{-A \eta v h\left(1+\lambda^{2}\right)}{2 E^{*} \cos \gamma}-\frac{b \tan \gamma}{v h}\right\} \\
& +p_{\gamma}\left\{\frac{A h \eta \lambda}{\cos \gamma}+1-\frac{b}{h v^{2}}\right\} .
\end{aligned}
$$

Using the first order optimal condition with respect to the lift control $\lambda, \mathcal{H}$ is maximized when $d \mathcal{H} / d \lambda=0$ (see Eq.(3.6)), which leads to

$$
\lambda=\frac{E^{*} p_{\gamma}}{v p_{v}} .
$$

From the second order optimal condition, the second derivative of $\mathcal{H}$ with respect to $\lambda$ should be negative to satisfy Pontryagin's maximum principle [see Eq.(3.7)]; this happens when $|\gamma|<90^{\circ}$ and $p_{v}>0$.

By using Eq.(3.9), the adjoint variables satisfy the necessary conditions

$$
\frac{d p_{v}}{d \theta}=-\frac{\partial \mathcal{H}}{\partial v} ; \frac{d p_{\gamma}}{d \theta}=-\frac{\partial \mathcal{H}}{\partial \gamma} ; \frac{d p_{h}}{d \theta}=-\frac{\partial \mathcal{H}}{\partial h} .
$$

By introducing a new parameter $C=p_{h} / p_{v}$, differentiating $\lambda$ and $C$ with respect to $\theta$ and using Eq.(4.11) we have, for $n=2$,

$$
\begin{aligned}
\frac{d \lambda}{d \theta}= & \frac{-E^{*} h C}{v \cos ^{2} \gamma}+\frac{A \eta h \sin \gamma\left(1-\lambda^{2}\right)}{2 \cos ^{2} \gamma}+\frac{E^{*} b}{h v^{2} \cos ^{2} \gamma} \\
& +\frac{2 \lambda b \tan \gamma}{h v^{2}}+\frac{2 \lambda^{2} b}{E^{*} h v^{2}}
\end{aligned}
$$




$$
\begin{aligned}
\frac{d C}{d \theta}= & \frac{A \eta v\left(1-\lambda^{2}\right)\left(1-h r_{o} z\right)}{2 E^{*} \cos \gamma}-\frac{b \tan \gamma}{h^{2} v}-C \tan \gamma \\
& -\frac{\lambda b}{E^{*} h^{2} v}-\frac{A \eta h C\left(1+\lambda^{2}\right)}{2 E^{*} \cos \gamma} \\
& +\frac{C b \tan \gamma}{h v^{2}}+\frac{2 C b \lambda}{E^{*} h v^{2}} .
\end{aligned}
$$

One can now obtain the modified Hamiltonian in terms of $\lambda$ and $C$ as

$$
\begin{aligned}
\hat{\mathcal{H}}= & \frac{\mathcal{H}}{p_{v}}=\frac{A \eta v h\left(\lambda^{2}-1\right)}{2 E^{*} \cos \gamma}-\frac{b \tan \gamma}{h v}+\frac{\lambda v}{E^{*}} \\
& -\frac{\lambda b}{E^{*} h v}+C h \tan \gamma .
\end{aligned}
$$

If $n$ is taken as 1.5 in the drag polar, then Eqs.(4.12)-(4.14) become

$$
\begin{aligned}
\frac{d \lambda}{d \theta}= & -\frac{2 E^{*} h C|\lambda|^{1 / 2}}{v \cos ^{2} \gamma}+\frac{2 A|\lambda|^{1 / 2} \eta h \sin \gamma\left(1-|\lambda|^{3 / 2}\right)}{3 \cos ^{2} \gamma} \\
& +\frac{2|\lambda|^{1 / 2} E^{*} b}{h v^{2} \cos ^{2} \gamma}+\frac{4 \lambda b \tan \gamma}{h v^{2}}+\frac{4|\lambda|^{3 / 2} b}{E^{*} h v^{2}}, \\
\frac{d C}{d \theta}= & \frac{A \eta v\left(1-|\lambda|^{3 / 2}\right)\left(1-h r_{o} z\right)}{3 E^{*} \cos \gamma}-\frac{b \tan \gamma}{h^{2} v}-C \tan \gamma \\
& -\frac{|\lambda|^{1 / 2} \operatorname{sgn}(\lambda) b}{E^{*} h^{2} v}-\frac{A \eta h C\left(1+2|\lambda|^{3 / 2}\right)}{3 E^{*} \cos \gamma} \\
& +\frac{C b \tan \gamma}{h v^{2}}+\frac{2 C b|\lambda|^{1 / 2} \operatorname{sgn}(\lambda)}{E^{*} h v^{2}} \\
\hat{\mathcal{H}}= & \frac{\mathcal{H}}{p_{v}}=C h \tan \gamma-\frac{A \eta v h\left(|\lambda|^{3 / 2}+1\right)}{3 E^{*} \cos \gamma}-\frac{b \tan \gamma}{h v} \\
& +\frac{|\lambda|^{1 / 2} \operatorname{sgn}(\lambda) v}{E^{*}}-\frac{|\lambda|^{1 / 2} \operatorname{sgn}(\lambda) b}{E^{*} h v} \\
& +\frac{A \eta h v \lambda|\lambda|^{1 / 2} \operatorname{sgn}(\lambda)}{E^{*} \cos \gamma} .
\end{aligned}
$$

while the lift control is given by

$$
|\lambda|=\left(\frac{E^{*} p_{\gamma}}{v p_{v}}\right)^{2}
$$

From Sec. 3.1.2, we know that the optimal solution needs transversality conditions to be satisfied while maximizing a functional or performance index involving the final 
values of the state variables. In our case these conditions are [see Eq.(3.13)]

$$
p_{\theta_{f}}=\mathcal{H}=\frac{\partial J}{\partial \theta_{f}} ; p_{v_{f}}=\frac{\partial J}{\partial v_{f}} ; p_{\gamma_{f}}=\frac{\partial J}{\partial \gamma_{f}} \text {. }
$$

Now the transversality condition to be satisfied by the normalized lift coefficient is

$$
\lambda_{f}=\frac{E^{*} p_{\gamma_{f}}}{v_{f} p_{v_{f}}}, \text { for } n=2,
$$

and

where

$$
|\lambda|_{f}=\left(\frac{E^{*} p_{\gamma_{f}}}{v_{f} p_{v_{f}}}\right)^{2}, \text { for } n=1.5
$$

$$
\begin{aligned}
& p_{v_{f}}=\frac{v_{f}\left(v_{\theta} \cos \epsilon+v_{r} \sin \epsilon\right)}{v_{s} v_{\infty}^{+}}+\frac{\partial \delta}{\partial v_{f}} \frac{v_{\infty}^{+}\left(v_{\theta} \sin \epsilon-v_{r} \cos \epsilon\right)}{v_{s}} \\
& p_{\gamma_{f}}=\frac{v_{\infty}^{+}\left(v_{\theta} \sin \epsilon-v_{r} \cos \epsilon\right)}{v_{s}} \frac{\partial \delta}{\partial \gamma_{f}} \\
& p_{\theta_{f}}=\frac{v_{\infty}^{+}\left(v_{\theta} \sin \epsilon-v_{r} \cos \epsilon\right)}{v_{s}}
\end{aligned}
$$

The values of $\partial \delta / \partial v_{f}$ and $\partial \delta / \partial \gamma_{f}$ are quite lengthy and therefore are omitted here for brevity, but can be found in Appendix A.

We have a second transversality condition for our solution, because the final value of the independent variable is not free and included in the performance index, hence $\hat{\mathcal{H}} \neq 0$ (see Sec. 3.1.1). Therefore the modified Hamiltonian $\hat{\mathcal{H}}$ satisfies the second transversality condition

$$
\hat{\mathcal{H}}_{f}-\frac{p_{\theta_{f}}}{p_{v_{f}}}=0 .
$$

Now we have five equations, namely Eqs.(4.2) for the three states, and either Eqs.(4.12)-(4.13) or Eqs.(4.15)-(4.16), depending on $n$. Integration of these equations will yield extremal trajectories for a number of situations which differ only in the entry and exit conditions that must be satisfied. We have a two-point boundary value problem, since the states $h, v, \gamma$ satisfy specified initial conditions, while $h$ and the co-states $p_{v}, p_{\gamma}$ must satisfy conditions specified at $\theta=\theta_{f}$. Our problem is also a two parameter problem because $\hat{\mathcal{H}} \neq 0$ and we have to guess the initial values of $C$ and $\lambda$ during the course of solving the two-point boundary value problem.

Although the heating rate constraint is not imposed on the solution in this Chapter, it will be calculated to see if it becomes excessive. 


\subsection{Method of Solution}

Calculations were carried out with specified values of $V_{\infty}^{-}$. Different values of $V_{\infty}^{-}$ imply different heliocentric trajectories from the Earth to the planet. From these, the entrance conditions $v_{e}$ and $\gamma_{e}$ can be computed. We have a two-point boundary value problem resulting from the optimization as shown in Sec. 3.2 and the system of ODEs in the form of Eq.(3.19) which can be solved by the shooting method. Using the computed values of $v_{e}$ and $\gamma_{e}$, setting $h_{e}=R / r_{o}$ and initial guesses for $\lambda_{e}$ and $C_{e}$ as initial conditions, Eqs. (4.2), (4.12) and (4.13) or Eqs.(4.2), (4.15) and (4.16) are integrated from $\theta=0$ until $h$ becomes $R / r_{o}$ again. The integration is performed by the Adams-Moulton method up to order twelve, with local absolute error controlled to be less than $1.0 \times 10^{-8}$. In all the cases studied, it has been possible to find a set of initial guess values of $\lambda_{e}$ and $C_{e}$ such that boundary conditions are satisfied at the exit after the iterations involved in the shooting method.

The trajectory and control computed are optimal, since, the necessary conditions \{Eqs.(4.2), (4.12) and (4.13)\}, the entry and exit conditions \{Eqs.(4.7), and (4.8)\}, and the transversality conditions Eqs.(4.20) and (4.23) are satisfied. There are more than one local maxima but the global maximum can be identified by examining the atmospheric trajectory. For example, in the case of global maximum, $\theta_{f}$ must be significantly large and the kinetic energy loss must be small, i.e. the vehicle should fly close to $E^{*}$ during most of the atmospheric trajectory. It was noted that the tolerance in satisfying the exit boundary condition has a negligible effect on the optimal value of $V_{s}^{+}$.

\subsection{Validation}

Before discussing the results, the accuracy of the formulation and the computer program must be checked. In order to do so, we consider an aeroassisted orbital transfer (AOT) problem, which is different from the problem considered here, but is somewhat similar. This AOT problem has been considered by Mease and Vinh (1985). We solve it by modifying the optimal necessary conditions developed above, and then 
compare the results with those of Mease and Vinh.

In this problem, the geocentric orbit of OTV (orbital transfer vehicle) is changed to a coplanar Low-Earth-Orbit by using aerodynamic maneuvering. Mease and Vinh have used time as the independent variable in the equations of motion for while in our case $\theta$ is the independent variable. But the dimensional state variables $r, V$, and $\gamma$ are the same, hence the difference in the independent variable will not affect the validation; of course, the aero-gravity assist problem must be replaced by the orbital transfer problem. For this problem, we have the initial state variables as

$$
V_{e}=10.32 \mathrm{~km} / \mathrm{s}, \quad \gamma_{e}=6.4 \mathrm{deg}, \text { and } r_{e}=R=6498 \mathrm{~km}
$$

and the conditions to be satisfied at the end of the atmospheric maneuvering are

$$
\gamma_{f}=0.4 \mathrm{deg}, r_{f}=R \text {. }
$$

The final time is free. Thus, $\hat{\mathcal{H}}=0$ and the value of $C_{e}$ can be calculated from Eq.(4.14).

Figure 4.1 shows reasonable agreement between the results obtained by using our optimality conditions and computer program and those of Mease and Vinh, although the independent variables in both cases are different and an exponential model to approximate the variation of the atmospheric density with the altitude is used here while Mease and Vinh have used a density model based on Chebyshev polynomials. This comparison gives us some confidence in the accuracy of the formulation.

\subsection{Results and Discussion}

For all the cases discussed below, the planet chosen for AGA is either Venus or Mars and the transfer is from $H_{T_{1}}$ to $H_{T_{2}}$, which will change the heliocentric trajectory of the spacecraft at a heliocentric distance of $1.084 \times 10^{8} \mathrm{~km}$ for Venus or $2.27 \times 10^{8} \mathrm{~km}$ for Mars, from the Sun, i.e., at the mean distance of the relevant planet from the Sun. The radius of the atmosphere, $R$, is taken as 6190 or $3483 \mathrm{~km}$ for Venus and Mars, respectively (Note that the radius of Venus is $6050 \mathrm{~km}$ and that for Mars is $3383 \mathrm{~km}$ ). It has been observed in AOT problems that variation in the radius of the sensible 
Present Results
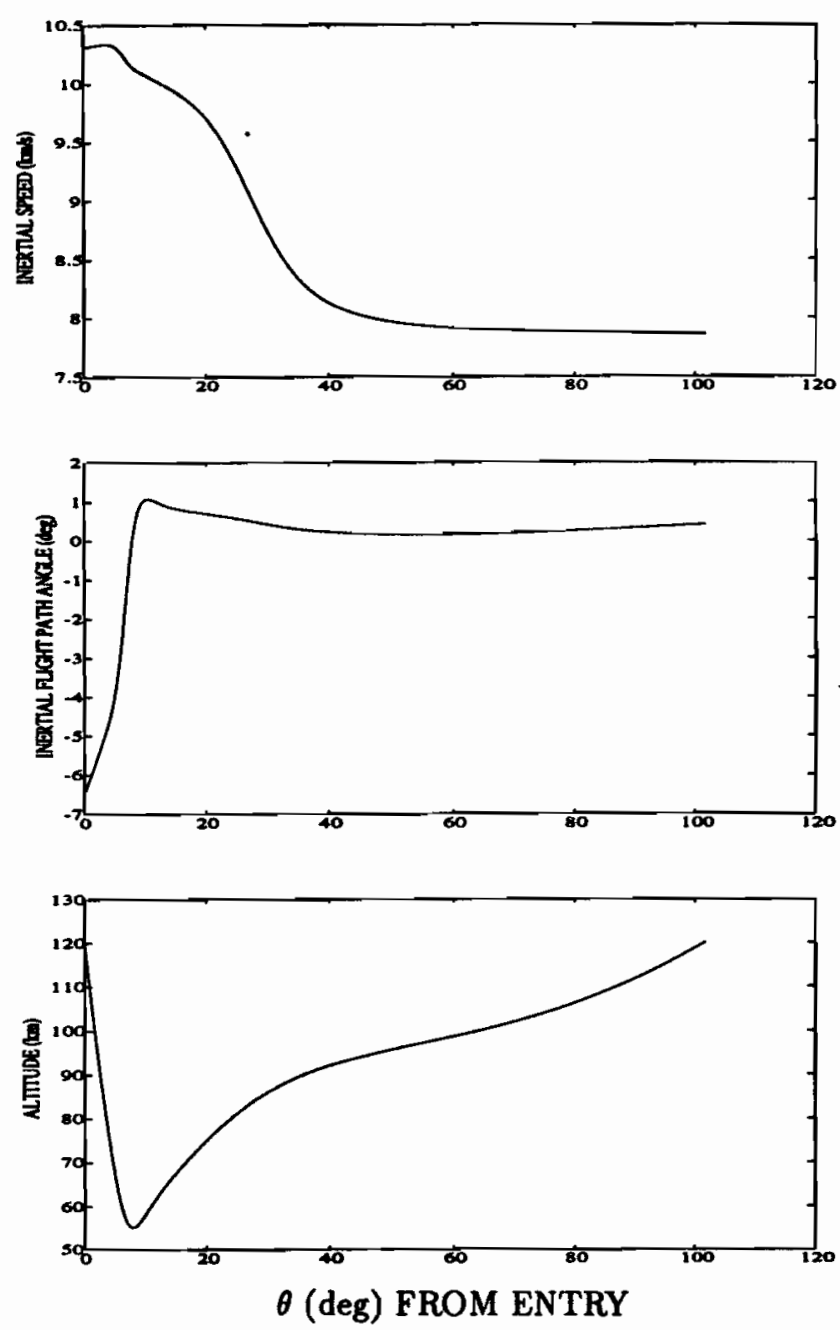

Results of Mease and Vinh
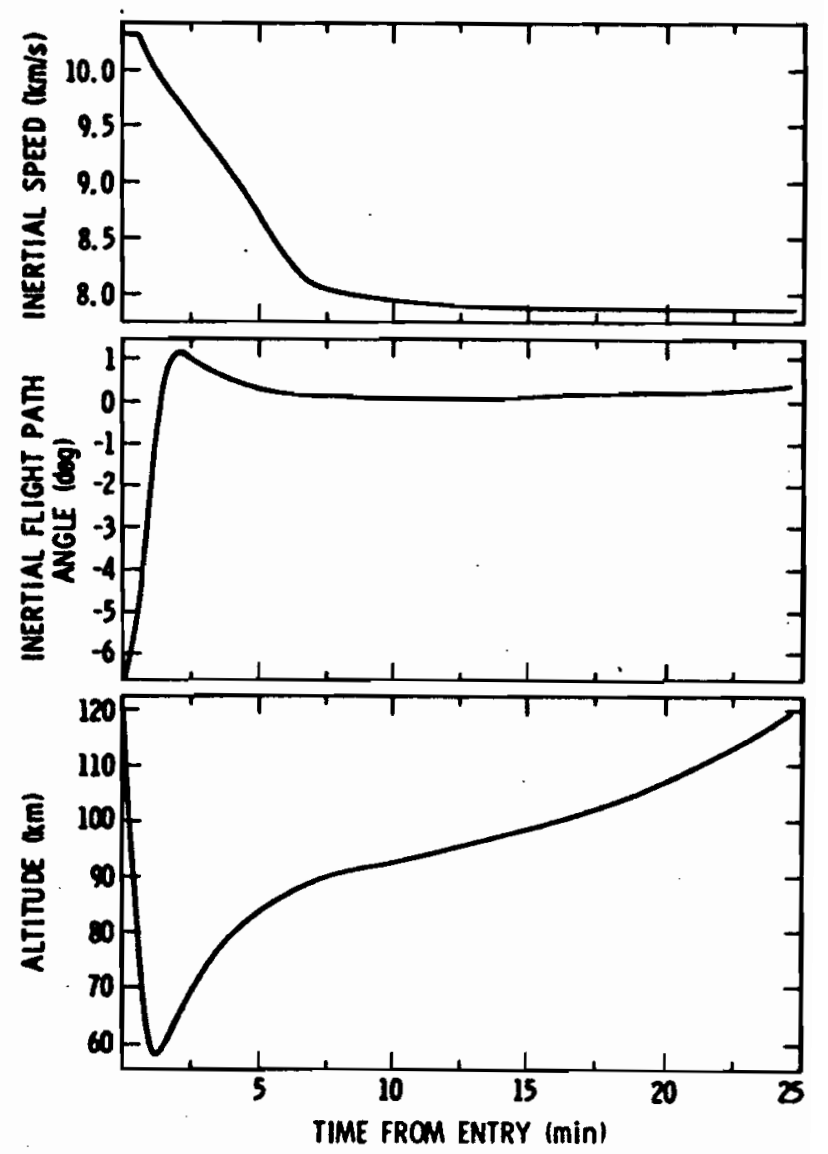

Figure 4.1: Comparison of the variation of the state variables with $\theta$ as obtained here with those given by of Mease and Vinh (1985). 
atmosphere $R$ does not affect the atmospheric trajectory much (Mease and Vinh 1985). Similar behavior is expected in AGA as well. Hence, the radius of the sensible atmosphere was assigned a reasonable value and was not varied. Above this altitude, the atmospheric density was assumed to be identically zero. Within the assumed atmospheric flight altitude range, $40-140 \mathrm{~km}$ for Venus and $20-100 \mathrm{~km}$ for Mars, the density was approximated by a model in which the density reduces exponentially with the increase in altitude. The scale height $S_{h}$ defined earlier was calculated according to the atmospheric data of Venus and Mars presented by Kondratyev and Hunt (1982) and Seiff (1991). The vehicle mass-to-surface area is $50 \mathrm{~kg} / \mathrm{m}^{2}$ for all the cases, except the case when we compare Mars-AGA and Venus-AGA.

The results of the analysis are presented in five parts. In the first part, we compare $V_{s}^{+}$obtained from the simple Venus-GA with that obtained from the optimal VenusAGA. In the second part, we discuss the optimal atmospheric trajectories for $n=2$ and 1.5. In the third part, we compare the $V_{s}^{+}$resulting from optimal AGA with different values of $E^{*}$. In the fourth part, the effect of $C_{L}^{*}$ on the heating rates is examined. Finally, Mars-AGA and Venus-AGA are compared.

\subsubsection{Comparison of GA with AGA}

We have taken three values of $V_{\infty}^{-}$, i.e. 10,12 , and $14 \mathrm{~km} / \mathrm{s}$, and corresponding results for Venus-AGA are shown in Fig.4.2 along with a plot for simple gravity assist. We have assumed that $E^{*}=5$ and $C_{L}^{*}=0.3$ for the vehicle at hypersonic velocity. The AGA results for the values of $C_{L}^{*}$ and $E^{*}$ for some Waverider hypersonic vehicle configurations developed by Anderson (1992) are given in Table 4.3. To obtain the results for GA, we have chosen the distance of closest approach to be $r_{p}=6550 \mathrm{~km}$, which is well above the radius of the atmosphere. It is clear from Fig.4.2 that AGA is superior to simple GA. It also shows that as planetocentric velocity $V_{\infty}^{-}$increases it leads to a smaller heliocentric velocity $V_{s}^{+}$with $\mathrm{GA}$.

One might argue that the $\Delta V$ for GA is lower partly because the distance of closest approach is greater compared to AGA. However, this difference in $r_{p}$ has only a negligible effect. In Table $4.1, V_{s}^{+}$for simple GA has been calculated using the same 
$r_{p}$ as in AGA and assuming that there is no atmosphere. The difference between $V_{s}^{+}$ for GA in Table 4.1 and Fig.4.2 is negligible and AGA retains its superiority.

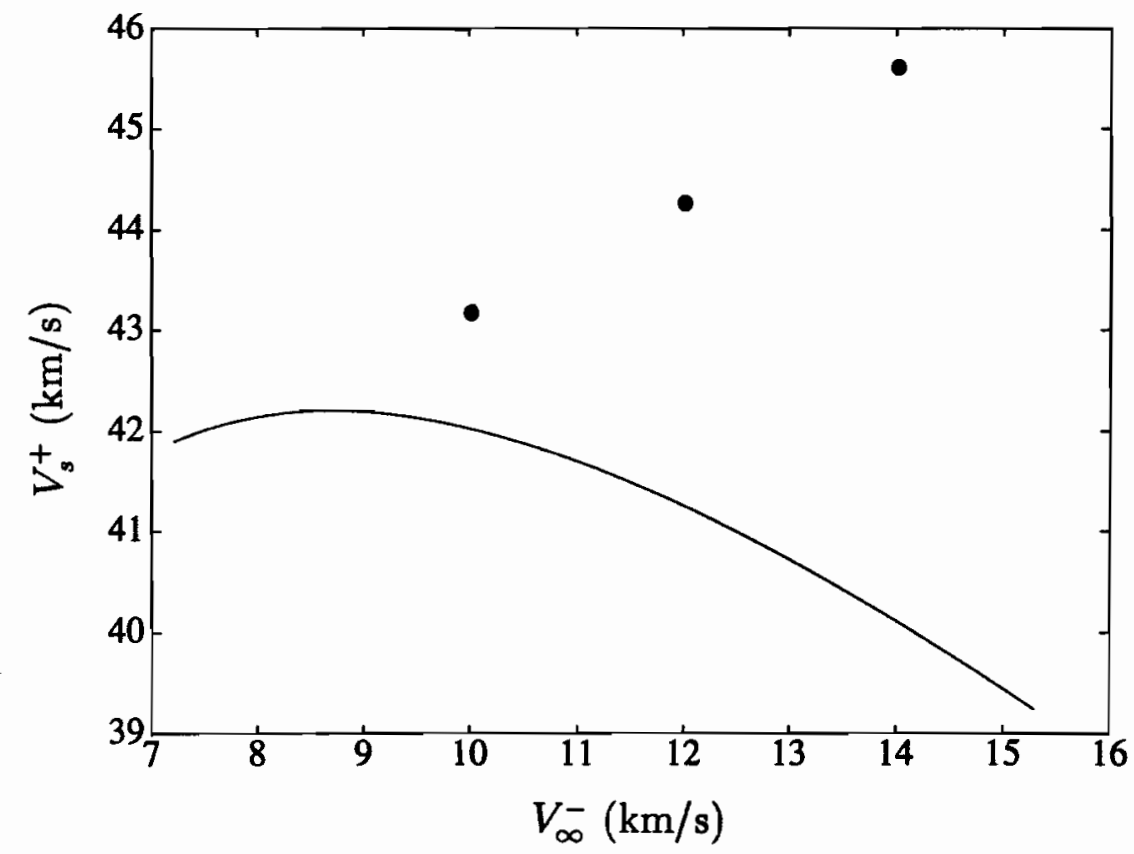

Figure 4.2: Variation of $V_{s}^{+}$vs $V_{\infty}$ for GA and AGA; $\bullet$ for AGA and solid line for GA.

Table 4.1 also presents some detailed results for AGA for three different values of $V_{\infty}^{-}$. An important quantity listed in judging the effectiveness of AGA (or GA) is $\Delta V_{E} ;$ as defined in Sec. 2.2 it is the $\Delta V$ needed at a Low-Earth-Orbit to send the spacecraft to Venus to arrive with a given $V_{s}^{-}$. Note that $V_{s}^{+}$increases with $V_{\infty}^{-}$at Venus; however, a large $V_{\infty}^{-}$implies a large $\Delta V_{E}$ as well, thereby reducing the total gain.

It can be noticed from Table 4.1 that $\delta_{2}$ is greater than $\delta_{1}$; this happens because the loss of kinetic energy of the spacecraft due to the drag during maneuvering through the planetary atmosphere causes a decrease in the eccentricity $e_{2}$. Table 4.1 also shows that $\theta_{f}$ for AGA increases with an increase in $V_{\infty}^{-}$leading to greater $V_{s}^{+}$. This happens because the sum of $\delta_{1}$ and $\alpha$ decreases with an increase in $V_{\infty}^{-}$. Similarly, the difference between $V_{s}^{+}$for AGA and GA decreases with a decrease in $V_{\infty}^{-}$, because at smaller $V_{\infty}^{-}$, e.g. $V_{\infty}^{-}<10 \mathrm{~km} / \mathrm{s}, \cos \epsilon$ becomes close to 1 and there is not much 
Table 4.1: Variation of Venus-AGA Results with $V_{\infty}^{-}$

\begin{tabular}{lrrr}
\hline$V_{\infty}^{-}(\mathrm{km} / \mathrm{s})$ & 10.00 & 12.00 & 14.00 \\
\hline \hline$V_{\infty}^{+}(\mathrm{km} / \mathrm{s})$ & 8.31 & 9.34 & 10.83 \\
$V_{s}^{-}(\mathrm{km} / \mathrm{s})$ & 36.20 & 35.54 & 34.83 \\
$V_{s}^{+}(\mathrm{km} / \mathrm{s})$ & 43.14 & 44.23 & 45.58 \\
$V_{s}^{+}(\mathrm{GA})(\mathrm{km} / \mathrm{s})$ & 42.03 & 41.36 & 40.37 \\
$\delta_{1} / 2($ deg. $)$ & 20.23 & 15.57 & 12.25 \\
$\delta_{2} / 2($ deg. $)$ & 25.70 & 22.17 & 18.10 \\
$\theta_{f}($ deg. $)$ & 46.75 & 63.48 & 69.21 \\
$\beta_{s}^{-}($deg. $)$ & 16.10 & 19.53 & 23.09 \\
$\beta_{s}^{+}($deg. $)$ & 2.86 & 2.45 & 3.60 \\
$\Delta V_{E}(\mathrm{~km} / \mathrm{s})$ & 4.14 & 4.51 & 5.04 \\
$\lambda_{e}$ & 3.02 & 1.93 & 0.59 \\
$\gamma_{e}($ deg. $)$ & -6.00 & -6.00 & -6.00 \\
for $r_{n}=1 \mathrm{~m}$ & & & \\
$q_{c_{\text {max }}}\left(\mathrm{W} / \mathrm{cm}^{2}\right)$ & 361.47 & 464.71 & 611.45 \\
$q_{r_{\text {max }}}\left(\mathrm{W} / \mathrm{cm}^{2}\right)$ & 272.23 & 398.37 & 523.00 \\
for $r_{n}=0.5 \mathrm{~m}$ & & & \\
$q_{c_{\text {max }}}\left(\mathrm{W} / \mathrm{cm}^{2}\right)$ & 511.20 & 657.20 & 864.72 \\
$q_{r_{\max }}\left(\mathrm{W} / \mathrm{cm}^{2}\right)$ & 187.23 & 274.00 & 359.70 \\
\hline
\end{tabular}


benefit of AGA. Hence AGA of Venus is advantageous only when $V_{\infty}^{-} \geq 10 \mathrm{~km} / \mathrm{s}$. Examining in Table 4.1 the values of $\beta_{s}$ before and after AGA, it is clear that after AGA $\beta_{s}$ is reduced significantly, which means that the perihelion distance of the spacecraft becomes quite close to the distance of Venus from the Sun. It may also be noted that $\lambda_{e}$ decreases (while $\gamma_{e}$ is constant) with increasing $V_{\infty}^{-}$, which increases the entrance velocity $V_{e}$. This is logical because higher $V_{e}$ needs less lift force in the beginning to bring the spacecraft to an appropriate altitude for maneuver.

\subsubsection{Nature of the Atmospheric Trajectory}

Figures 4.3 and 4.4 show the variation of $v, \gamma, h$ and $\lambda$ with $\theta$ for the optimal atmospheric trajectory obtained for the two cases of $n=2$ and $n=1.5$ for the planet Venus. Solid and dashed lines show the time history of the variables for $n=2$ and $n=1.5$, respectively. We have taken here $V_{s}^{-} \cos \beta_{s}^{-}=34 \mathrm{~km} / \mathrm{s}$; corresponding values of $V_{\infty}^{-}$is $11.24 \mathrm{~km} / \mathrm{s}, E^{*}=5$ and $C_{L}^{*}=0.3$. We can see that most of the time the vehicle stays close to $\lambda=-1$ (Fig. 4.4), which means $L / D=E^{*}$, so as to limit the loss of kinetic energy. It maintains a constant flight path angle $\gamma$ close to zero. One can also observe the variation of altitude versus $\theta$ in Fig. 4.3 which reveals that after the vehicle enters the atmosphere, it lowers itself to an almost circular trajectory. However, it is not truly circular. As the velocity reduces due to the drag, its altitude increases slightly to maintain $\lambda=-1$. Hence during that portion of the atmospheric trajectory when $\lambda=-1$, the path of the vehicle is more like part of a spiral than circular. $V_{s}^{+}$for $n=2$ and $n=1.5$ are approximately the same after AGA although $\theta_{f}$ for the two cases are different. This is because the main part of the atmospheric trajectory is nearly identical for the two cases. With imposition of a heat constraint, however, the two values of $V_{s}^{+}$will be different, as we will see in Chapter 5 .

\subsubsection{Effect of $E^{*}$ on Optimal $V_{s}^{+}$}

The value of $E^{*}$ was changed to see how it affects the maximum $V_{s}^{+}$after Venus-AGA. The entrance flight path angle $\gamma_{e}$ is $-6^{\circ}$. We can see from the first two columns of 

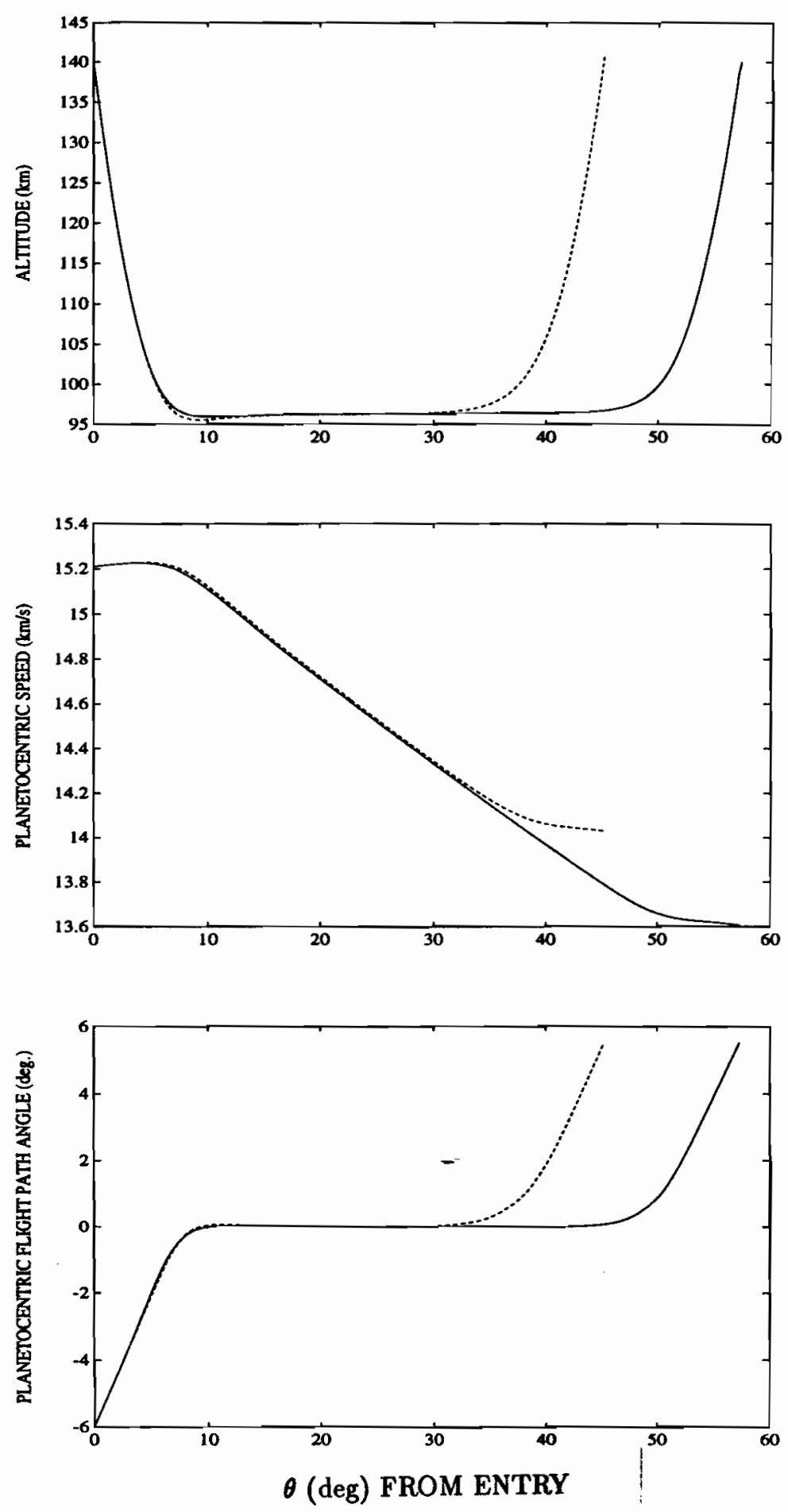

Figure 4.3: Variation of the state variables with $\theta$. Solid and dashed lines are for $n=2$ and $n=1.5$, respectively. 


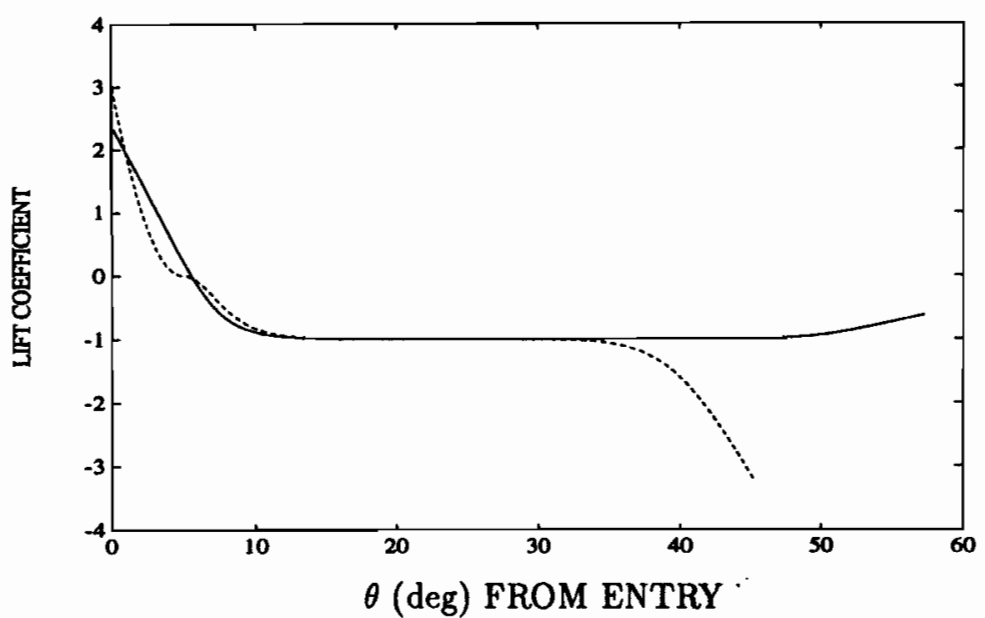

Figure 4.4: Variation of normalized lift coefficient $\lambda$ with $\theta$. Solid and dashed lines are for $n=2$ and $n=1.5$, respectively.

Table 4.2 that for lower $E^{*}, \theta_{f}$ is smaller so as to limit the loss of kinetic energy, which decreases $V_{s}^{+}$compared to the case of higher $E^{*}$. However, even for a small $E^{*}(=1.5)$, the optimal $V_{s}^{+}$can still be significantly higher than $V_{s}$ in the case of simple GA, provided $V_{\infty}^{-}$is sufficiently high; for example, in the case of $V_{\infty}^{-}=14 \mathrm{~km} / \mathrm{s}$ in column 4 of Table 4.2. Table 4.2 also shows that if we double $E^{*}$ then $V_{s}^{+}$can be increased by more than $1 \mathrm{~km} / \mathrm{s}$.

\subsubsection{Effect of $C_{L}^{*}$ on the Heating Rates}

Next we analyse the effect of $C_{L}^{*}$ on the heating rate. $V_{\infty}^{-}$is $11.24 \mathrm{~km} / \mathrm{s}$ and the planet considered is Venus for all the cases. First, we use two values of $C_{L}^{*}, 0.0152$ and 0.0342 , given in a study by Anderson et. al. (1990) for Waverider configurations for AGA. These values of $C_{L}^{*}$ are for Mach numbers 30 and 19, respectively, but let us assume that a slightly different vehicle can have the same $C_{L}^{*}$ and $E^{*}$ at higher Mach numbers, say $M>50$. We can see from Table 4.3 that the maximum heating rates during maneuvering through the atmosphere are very high for these configurations, particularly the radiative heating rate. This is because a small $C_{L}^{*}$ leads to a lower altitude to maneuver at $\lambda=-1$, as shown in the last row in Table 4.3, thus higher 
Table 4.2: Effect of $E^{*}$ on $V_{s}^{+}$

\begin{tabular}{lrr|rr}
\hline$E^{*}$ & 10.00 & 5.00 & 3.00 & 1.5 \\
\hline \hline$C_{L}^{*}$ & 0.30 & 0.30 & 0.30 & 0.30 \\
$V_{\infty}^{-}(\mathrm{km} / \mathrm{s})$ & 11.24 & 11.24 & 14.00 & 14.00 \\
$V_{\infty}^{+}(\mathrm{km} / \mathrm{s})$ & 9.93 & 8.95 & 10.07 & 9.61 \\
$V_{s}^{+}(\mathrm{km} / \mathrm{s})$ & 44.85 & 43.82 & 44.73 & 42.49 \\
$V_{s}^{+}(\mathrm{GA})(\mathrm{km} / \mathrm{s})$ & 41.71 & 41.71 & 40.37 & 40.37 \\
$\theta_{f}($ deg. $)$ & 62.57 & 57.30 & 61.50 & 42.40 \\
$\beta_{s}^{-}($deg. $)$ & 17.76 & 17.76 & 23.09 & 23.09 \\
$\beta_{s}^{+}($deg. $)$ & 2.34 & 2.63 & 4.60 & 7.50 \\
$\lambda_{e}$ & 2.39 & 2.38 & 0.60 & 0.62 \\
$\gamma_{e}($ deg. $)$ & -6.00 & -6.00 & -6.00 & -6.00 \\
\hline
\end{tabular}

density and heating rate. We can also notice that maneuvering of a vehicle with $C_{L}^{*}=0.3$ and $E^{*}=10$ gives significantly lower heating rates. Since the vehicle will have relatively thin wings for such a mission, therefore a vehicle has to be developed which not only has high $E^{*}$ but also high $C_{L}^{*}$ to reduce the heating. It can also be noticed that $\left|\gamma_{e}\right|$ is higher for the lower $C_{L}^{*}$ cases, because the vehicle in these cases is unable to reach an appropriate altitude for maneuvering at $\lambda=-1$. We can also see from Table 4.1 that increasing $V_{\infty}^{-}$from $10 \mathrm{~km} / \mathrm{s}$ to $14 \mathrm{~km} / \mathrm{s}$ does not increase the maximum heating rates drastically.

\subsubsection{Comparison of GA and AGA for Mars and Venus}

Now the two planets, Mars and Venus, are compared with regarding the effectiveness of their AGA. We have considered a spacecraft with $m / S C_{L_{\max }}=50 \mathrm{~kg} / \mathrm{m}^{2}$, which is the same as used by McRonald and Randolph (1992). For Venus, the difference between $V_{s}^{+}$for AGA and GA decreases with a reduction in $V_{\infty}^{-}$, because at smaller $V_{\infty}^{-}$, e.g. $V_{\infty}^{-}<10 \mathrm{~km} / \mathrm{s}, \cos \epsilon$ becomes close to 1 and there is not much benefit of 
Table 4.3: Effect of $C_{L}^{*}$ on the Heating Rates

\begin{tabular}{lrrr}
\hline$C_{L}^{*}$ & 0.0151 & 0.0342 & 0.30 \\
\hline \hline$E^{*}$ & 14.52 & 9.14 & 10.00 \\
$V_{\infty}^{-}(\mathrm{km} / \mathrm{s})$ & 11.24 & 11.24 & 11.24 \\
$V_{\infty}^{+}(\mathrm{km} / \mathrm{s})$ & 10.53 & 9.82 & 9.93 \\
$V_{s}^{+}(\mathrm{km} / \mathrm{s})$ & 45.62 & 44.61 & 44.85 \\
$V_{s}^{+}(\mathrm{GA})(\mathrm{km} / \mathrm{s})$ & 41.71 & 41.71 & 41.71 \\
$\lambda_{e}$ & 2.94 & 4.22 & 2.39 \\
$\gamma_{e}($ deg. $)$ & -7.00 & -7.00 & -6.00 \\
for $r_{n}=1 \mathrm{~m}$ & & & \\
$q_{c_{\max }}\left(\mathrm{W} / \mathrm{cm}^{2}\right)$ & 1894 & 1259 & 429 \\
$q_{r_{\max }}\left(\mathrm{W} / \mathrm{cm}^{2}\right)$ & 14162 & 5232 & 376 \\
for $r_{n}=0.5 \mathrm{~m}$ & & & \\
$q_{c_{\max }}\left(\mathrm{W} / \mathrm{cm}^{2}\right)$ & 2678 & 1780 & 607 \\
$q_{r_{\max }}\left(\mathrm{W} / \mathrm{cm}^{2}\right)$ & 9835 & 3633 & 261 \\
Min altitude $(\mathrm{km})$ & 81.85 & 85.77 & 96.20 \\
\hline
\end{tabular}


AGA (see Fig.4.5). Hence AGA of Venus is advantageous only when $V_{\infty}^{-} \geq 10 \mathrm{~km} / \mathrm{s}$. On the contrary, for Mars, the difference between $V_{s}^{+}$for AGA and GA does not disappear for a small $V_{\infty}^{-}$; this is because Mars has a significantly smaller mass than Venus which causes a very small $\delta$ after Mars-GA. It can also be seen from Table 4.4 that the value of aphelion $r_{a s}$ after AGA is much higher for the case of Mars because of its farther location from the Sun compared with Venus. Also, the heating rates for the case of Mars are much lower than those for the case of Venus, since $V_{e}$ is lower in the former case due to a lower planetary mass. Though Table 4.4 shows that $\Delta V_{E}$ is slightly smaller for Venus-AGA than that for Mars-AGA, this advantage of Venus-AGA is cancelled out due to higher heating rate and lower aphelion $r_{a}$ after AGA. Therefore, one can conclude that Mars is a more suitable candidate for AGA than Venus.

Table 4.4 also reveals that the angle $\alpha$ between $V_{\infty}^{-}$and $V_{p}$ is higher for Mars-AGA in most of the cases than that for Venus-AGA. $\theta_{f}$ for Mars-AGA is also larger than that for Venus-AGA, because the terms $\left(\delta_{1}+\delta_{2}\right) / 2$ for Mars-AGA, which is related to simple GA, is considerably lower than that for Venus-AGA. In order to maximize $V_{s}^{+}$, one needs smaller $\beta_{s}^{+}$such that $\cos \beta_{s}^{+} \approx 1$; thus the drawback of lower $\left(\delta_{1}+\delta_{2}\right) / 2$ for Mars-AGA is compensated with higher $\theta_{f}$. Moreover, $\left|\gamma_{e}\right|$ for Mars-AGA is higher than that for Venus due to the lower mass of Mars (which means lower gravitational force), and as a result of that higher atmospheric density is required in the lower altitude region of maneuvering (where $\lambda \approx-1$ ) so that a sufficient negative lift force is generated to make up for the lower gravitational force. In the optimal trajectory, the average density in the lower altitude region for Mars $\left(1.0 \times 10^{-4} \mathrm{~kg} / \mathrm{m}^{3}\right)$ is higher than that for Venus $\left(4.3 \times 10^{-5} \mathrm{~kg} / \mathrm{m}^{3}\right)$. 
Chap. 4]
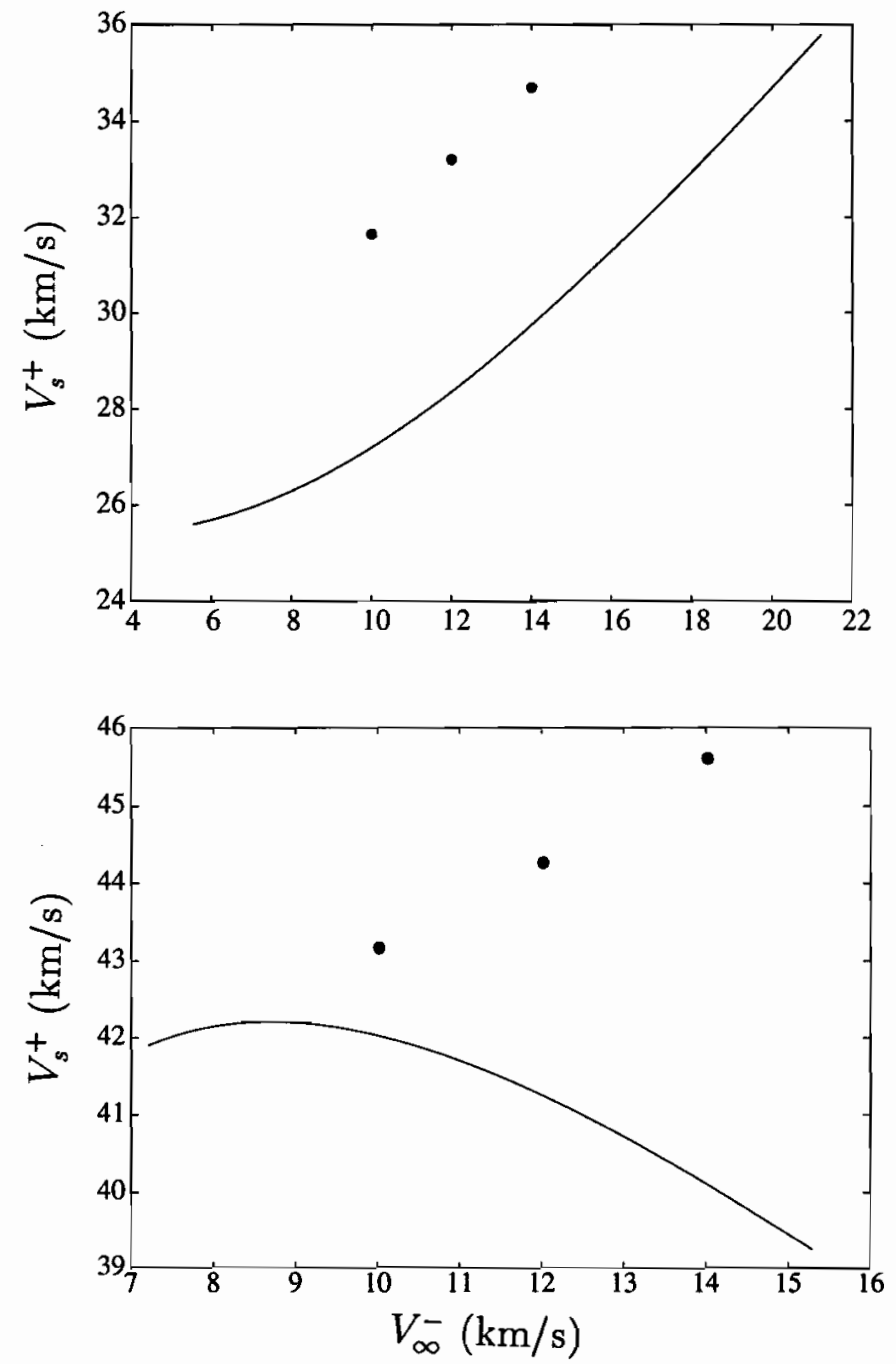

Figure 4.5: Variation of $V_{s}^{+}$vs $V_{\infty}^{-}$for GA and AGA for Mars and Venus; $\bullet$ for AGA and solid line for GA. 
Table 4.4: Comparison of Mars-AGA and Venus-AGA Results with $V_{\infty}^{-}$

\begin{tabular}{|c|c|c|c|c|c|c|}
\hline \multirow[b]{2}{*}{$V_{\mathrm{oo}}^{-}(\mathrm{km} / \mathrm{s})$} & \multicolumn{3}{|c|}{ results for Mars } & \multicolumn{3}{|c|}{ results for Venus } \\
\hline & 10.00 & 12.00 & 14.00 & 10.00 & 12.00 & 14.00 \\
\hline$V_{\infty}^{+}(\mathrm{km} / \mathrm{s})$ & 7.72 & 9.15 & 10.60 & 8.31 & 9.34 & 10.83 \\
\hline$V_{s}^{-}(\mathrm{km} / \mathrm{s})$ & 25.32 & 26.84 & 28.46 & 36.20 & 35.54 & 34.83 \\
\hline$V_{s}^{+}(\mathrm{km} / \mathrm{s})$ & 31.65 & 33.20 & 34.69 & 43.14 & 44.23 & 45.58 \\
\hline$V_{s}^{+}(\mathrm{GA})(\mathrm{km} / \mathrm{s})$ & 27.31 & 28.47 & 29.76 & 42.03 & 41.36 & 40.37 \\
\hline$\delta_{1} / 2($ deg. $)$ & 6.36 & 4.57 & 3.44 & 20.23 & 15.57 & 12.25 \\
\hline$\delta_{2} / 2$ (deg.) & 9.95 & 7.41 & 5.73 & 25.70 & 22.17 & 18.10 \\
\hline$\theta_{f}(\mathrm{deg})$. & 83.94 & 89.15 & 93.40 & 46.75 & 63.48 & 69.21 \\
\hline$\alpha(\mathrm{deg})$ & 84.80 & 89.50 & 92.63 & 87.94 & 82.08 & 79.57 \\
\hline$\beta_{s}^{-}($deg. $)$ & 23.25 & 26.62 & 29.42 & 16.10 & 19.53 & 23.09 \\
\hline$\beta_{s}^{+}(\operatorname{deg})$. & 3.72 & 2.53 & 2.16 & 2.86 & 2.45 & 3.60 \\
\hline$\Delta V_{E}(\mathrm{~km} / \mathrm{s})$ & 4.51 & 5.04 & 5.72 & 4.14 & 4.51 & 5.04 \\
\hline$V_{\infty_{E}}(\mathrm{~km} / \mathrm{s})$ & 5.50 & 6.60 & 7.84 & 4.63 & 5.50 & 6.61 \\
\hline$r_{a s}\left(10^{9} \mathrm{~km}\right)$ & 2.72 & 7.45 & $\infty$ & 0.67 & 0.84 & 1.12 \\
\hline$\lambda_{e}$ & 0.32 & -0.33 & 2.40 & 3.02 & 1.93 & 0.59 \\
\hline$\gamma_{e}$ (deg.) & -9.00 & -9.00 & -10.00 & -6.00 & -6.00 & -6.00 \\
\hline$V_{e}(\mathrm{~km} / \mathrm{s})$ & 11.16 & 12.98 & 14.85 & 14.32 & 15.78 & 17.35 \\
\hline for $r_{n}=1 \mathrm{~m}$ & & & & & & \\
\hline$q_{c_{\max }}\left(\mathrm{W} / \mathrm{cm}^{2}\right)$ & 223.65 & 351.43 & 586.78 & 361.47 & 464.71 & 611.45 \\
\hline$q_{r_{\max }}\left(\mathrm{W} / \mathrm{cm}^{2}\right)$ & 18.10 & 356.01 & 816.42 & 272.23 & 398.37 & 523.00 \\
\hline for $r_{n}=0.5 \mathrm{~m}$ & & & & & & \\
\hline$q_{c_{\max }}\left(\mathrm{W} / \mathrm{cm}^{2}\right)$ & 316.29 & 497.00 & 829.83 & 511.20 & 657.20 & 864.72 \\
\hline$q_{r_{\max }}\left(\mathrm{W} / \mathrm{cm}^{2}\right)$ & 12.38 & 244.85 & 561.51 & 187.23 & 274.00 & 359.70 \\
\hline
\end{tabular}




\section{Chapter 5}

\section{Optimal Aero-Gravity Assist with Heat Constraint}

In the previous chapter, we addressed the problem of augmenting gravity assist by using planetary atmospheric maneuvering such that the heliocentric velocity $V_{s}^{+}$of the spacecraft was maximized. The results showed that the heating rate for higher $V_{\infty}^{-}$ is fairly high. In this chapter, a heating rate constraint is imposed on the atmospheric trajectory for AGA with high $V_{\infty}^{-}$. Firstly, we will discuss the method of solution for obtaining an optimal atmospheric trajectory for AGA with a heating rate constraint. Secondly, we will compare the atmospheric trajectories for $n=2$ and 1.5 for the two cases of presence and absence of a heating rate constraint. We will also present a brief comparison between some important parameters related to the atmospheric trajectories with heating rate constraint for AOT (aero-assisted orbit transfer) and AGA. Finally, a sensitivity study is presented analyzing the effect of fluctuation of the atmospheric density.

\subsection{Method of Solution}

Before discussing the method used in the thesis for optimization of the atmospheric trajectory for AGA with a heating rate constraint, we will discuss briefly the approaches used in the previous research works to solve the problem of optimal atmo- 
spheric trajectory in the presence of a heating rate constraint.

\subsubsection{Inequality Constraints on Heating Rate}

The maximum heat transfer rate, the integrated heat or the average heat rate, and the maximum aero-dynamic acceleration are the important parameters that distinguish different atmospheric flight paths and dictate the thermal protection and structural mass requirements. Miele and his colleagues have conducted an extensive investigation (Miele et al. 1984, 1985, 1986, 1987) of a number of performance indices in order to determine which leads to the most favourable planar atmospheric flight path for changing terrestrial orbits. Among those performance indices two are as follows: (1) the integrated heat; (2) the peak heating rate. The heating rate is computed at the stagnation region. For each problem, the objective was to minimize the relevant performance index. The results of Miele's work show that using the second performance index gives almost identical atmospheric trajectory to that using the first performance index. Therefore, in this thesis we will impose a peak heating rate constraint on the atmospheric trajectory; consideration of intergrated heat will lead to similar results. Furthermore, we will not just minimize the performance index as Miele and his colleagues have done, instead we will impose an inequality heating rate constraint, that is the heating rate will be constrained to be less than a specified peak heating rate.

\section{Heating Rate Constraint}

Now one has to decide which heating rate (convective or radiative) to select in the optimization problem for the heating rate constraint. Although ideally the sum of the two should be used, it leads to implementation problem. Our calculations show that at low altitudes $q_{r}$ will be much higher than $q_{c}$, but at high altitudes $q_{r}$ is much lower than $q_{c}$. Since the altitude of maneuvering of the spacecraft in the present case will be high, therefore $q_{c}$ is selected to be used in the heating rate constraint. Moreover, if we impose a constraint on the heating rate then the integrated heat will automatically be reduced, although it may not be minimal. 
Recall that the convective heating rate, $q_{c}$, along the atmospheric trajectory was given in Chapter 2 as

$$
q_{c}=\frac{k \rho^{1 / 2} V^{3}}{\left(r_{n}\right)^{1 / 2}}
$$

where $q_{c}$ is in Watts $/ \mathrm{cm}^{2}$, while $k$ for Mars can be taken as $1.8425 \times 10^{-8}$. In the calculations we have chosen $r_{n}$ as either $0.5 \mathrm{~m}$ or $1 \mathrm{~m}$.

\section{Approaches to Solve the Problem with a Heating Rate Inequality Con- straint}

There are very few studies in which an inequality constraint was imposed on planar skip trajectories. One of them is the study by Mease and Vinh (1985); they have studied the aero-assisted orbit transfer problem by imposing an inequality peak heating rate constraint on the atmospheric trajectory. It may be noted that this is the same problem that was considered in Section 4.4 (Validation Section), but there it was solved without any heating rate constraint. The method used by Mease and Vinh to solve the problem with a peak heating rate constraint is given below briefly.

When a heating rate constraint is imposed, the solution procedure is somewhat different from that without such a constraint. Previous studies on skip trajectories have shown that the heating rate increases in a monotonic fashion reaching its maximum value shortly after the atmospheric entry. It then decreases during the remainder of the flight. In the approach followed by Mease and Vinh one begins at time zero, the goal being to choose a $\lambda_{\varepsilon}$ such that-at some later time, $q_{c}=q_{c_{\max }}$ and $\dot{q}_{c}=0$. When this state is achieved, then the second step is to find the values of $\lambda$ and $C$ such that boundary conditions are satisfied at the atmospheric exit. We tried to use this approach in our case, but after solving the first part, that is up to, $q_{c}=q_{c_{\max }}$ and $\dot{q}_{c}=0$, we found that convergence for the second part was possible only when the altitude reduced to that corresponding to the maximum $L / D$ ratio, and the heating rate constraint was violated. Hence, we concluded that this approach is difficult to implement for highly nonlinear boundary conditions. Thus we had to look for an alternative technique to solve our problem.

There exist some studies on optimal atmospheric trajectory with terrestrial plane 
change and optimal three-dimensional atmospheric reentry trajectory in which an inequality heating rate constraint was used. The simplest example of flight on the heating boundary is a constant altitude, constant speed trajectory, with the altitude and speed chosen such that $q_{c}=q_{c_{\max }}$. The speed is held constant by thrusting to cancel drag. This mode of flight is called aerocruise, as defined in Chapter 1. But we are not using thrust, therefore this approach can not be applied in our case either.

In a study conducted by Chern and his colleagues (1985), the following approach was used to solve the problem of three-dimensional atmospheric reentry flight of the shuttle with an inequality heating rate constraint. In this approach, the flight of the vehicle starts at $t=0$ and enters the boundary of a specified peak heating rate $q_{c_{\max }}$ such that

$$
q_{c}=q_{c_{\text {max }}} \text { and } \dot{q}_{c}=0 .
$$

Later the flight continues on the prescribed boundary of $q_{c_{\max }}$ for some time $t^{\prime}$ and then the vehicle leaves this boundary. The flight continues until some conditions are satisfied at specific speed of the spacecraft. The time $t^{\prime}$ is adjusted iteratively to satisfy the conditions.

A direct implementation of this approach is not possible here because in this approach the lift control is kept constant on the prescribed boundary and the bank angle is used as the control parameter, while in the present case the flight is planar and we have only one control parameter $\lambda$. However, the concept can be adapted to the present case so that it could be implemented successfully.

\subsubsection{Approach for Solving Optimal AGA Planar Problems with a Heating Rate Constraint}

In this approach the boundary conditions at the exit point B in Fig.2.4 remains the same as in Chapter 4 [Eqs(4.19-4.22)]; however, the problem is solved in parts. In the first part, we begin at $\theta=0$ as in the case of the unconstrained optimization, except that now the aim is to choose $\lambda_{e}$ and $C_{e}$ such that for some value of $\theta$ equal 
to $\hat{\theta}_{1}$,

$$
q_{c}=q_{c_{m a x}}, \frac{d \gamma}{d \theta}=0, \gamma=\gamma_{c}<0,
$$

where $\gamma_{c}$ is a specified flight path angle. The selection of $\gamma_{c}$ is made in such a way that the spacecraft can maneuver close to the boundary of $q_{c_{m a x}}$ in the second part. $\gamma$ remains constant, equal to $\gamma_{c}$, in the second part of the trajectory over an interval $\hat{\theta}=\hat{\theta}_{2}-\hat{\theta}_{1}$. When $\theta=\hat{\theta}_{2}$, the spacecraft leaves the prescribed boundary of $\gamma_{c}$. After that the spacecraft moves along the third trajectory, for which we choose $C$ at $\hat{\theta}_{2}$ such that the boundary conditions are satisfied at the point B in Fig.2.4 (at the altitude of the sensible atmosphere). In numerical intergration, what we actually do is adjust the instant of leaving the $\gamma_{c}$ boundary i.e, the value of $\hat{\theta}$, and check at the exit point if the boundary conditions are satisfied. Several iterations are required to attain this. In the interval $\hat{\theta}$ the control law is changed to

$$
\lambda=\left(\frac{b}{v^{2} h}-1\right) \frac{\cos \gamma}{A \eta h} .
$$

Thus, the function $C$ is discontinuous between the second and third components of the atmospheric trajectory, although the states $h, v, \gamma$ and the adjoint $\lambda$ are always continuous.

\subsection{Numerical Results and Discussion}

For the cases below, the planet Mars is used for AGA. It is assumed that $m / C_{L_{\max }} S=50$ $\mathrm{kg} / \mathrm{m}^{2}$ for all the cases, which is the same as that for some AGA missions being investigated at JPL ( $C_{L \max }$ stands for the maximum $C_{L}$ at stalling angle of attack). The global maximum is identified with the largest value of $V_{s}^{+}$among the local maxima.

The results of the analysis are presented in four parts: (1) Comparison of the optimal trajectories with the heat constraint for $n=2$, with those without heat constraint (in the absence of the heat constraint, the results for $n=2$ and $n=1.5$ are very close); (2) Comparison of $V_{s}^{+}$and other parameters for the above three cases; (3) Comparison of some parameters for AOT with those for AGA; (4) Sensitivity study on the aerodynamic maneuvering for AGA when there is an uncertainty in the knowledge of the atmospheric density. 


\subsubsection{Nature of the Atmospheric Trajectories without and with Heat Constraint}

Figure 5.1 shows the variation of $v, \gamma$ and $h$ with $\theta$ for the optimal atmospheric trajectories for the three cases: without heat constraint for $n=2$ and with heat constraint for $n=2$ as well as $n=1.5$. Since in the previous chapter, it was shown that the optimal atmospheric trajectories without heat constraint for $n=2$ and $n=1.5$ are nearly the same, therefore only one value of $n$ was considered here for that situation. Solid, dashed and dash-dotted lines show the time history for the three cases: in the absence of heat constraint, and with heat constraint for $n=2$ and 1.5, respectively. $V_{\infty}^{-}$was taken as $12 \mathrm{~km}$ with $r_{n}=1 \mathrm{~m}$ and $E^{*}=5$. The maximum heating rate constraint was chosen as $200 \mathrm{Watts} / \mathrm{cm}^{2}$. Since we want to reduce the heating rate as much as possible, the spacecraft should fly at nearly maximum lift coefficient $\lambda_{\max }$ when $q_{c}=q_{c_{\text {mas }}}, d \gamma / d \theta=0, \gamma=\gamma_{c}<0$. Without the heat constraint, we see that most of the time the vehicle stays close to $\lambda=-1$ as shown in Fig.5.2, which implies that the $L / D$ ratio is close to $E^{*}$ to limit the loss of kinetic energy; it also maintains a nearly constant $\gamma$ close to zero. For the same case, the variation of $h$ versus $\theta$ given in Fig.5.1 reveals that a few moments after the vehicle enters the atmosphere, the altitude of the vehicle becomes nearly constant, when $\lambda=-1$, which means that the vehicle flies in a path close to a circular one for some time, and then leaves the atmosphere.

The atmospheric trajectories for heat constraint cases with $n=2$ and $n=1.5$ are nearly identical, except the variation of $v$ versus $\theta$ which is different for the two cases. Figure 5.1 shows that the final velocity for the $n=1.5$ case is close to that without the heat constraint case, and is higher than the $n=2$ case. This is because the overall $L / D$ during the atmospheric trajectory is higher for $n=1.5$ compared to $n=2$ as shown in Fig.5.2. One can observe from Fig.5.1 that the vehicle flies most of the time with a constant flight path angle $\gamma_{c}$ turning the atmospheric trajectory into a spiral shape at the lower altitude.

One may ask why one should not use a circular path instead of a spiral one in the lower altitude region of the atmospheric trajectory, as was used in the earlier 
investigations (Randolph and McRonald 1989, 1992). Calculations show that $V_{s}^{+}$for a circular path will be lower than that for the spiral case, for example, for $V_{\infty}^{-}=12$ $\mathrm{km} / \mathrm{s}$ and $n=1.5$ for AGA with heat constraint, optimal $V_{s}^{+}$is $33.00 \mathrm{~km} / \mathrm{s}$, while for a circular path $V_{s}^{+}$is $32.23 \mathrm{~km} / \mathrm{s}$. After achieving the point where $q_{c}=q_{c_{\text {mas }}}$, $d \gamma / d \theta=0$ in the atmosphere for a circular path, the altitude remains constant i.e., $\gamma_{c}=0$, while $q_{c}$ and $|\lambda|$ decrease with the reduction in the planetocentric velocity due to the aerodynamic drag. Whereas in the case of a spiral path, $q_{c}$ remains constant and $|\lambda|$ decreases further because of the decreasing altitude (increasing density) and drag. Thus $|\lambda|$ is lower in the case of a spiral path than that for the circular path, and this leads to a higher overall $L / D$, and as a result the planetocentric velocity is higher for AGA after maneuvering in the atmosphere through the same $\hat{\theta}$.

Figure 5.3 shows that the heating rate and dynamic pressure are much lower in the presence of a heat constraint. Without the heat constraint, the dynamic pressure reaches a peak value a few moments after the vehicle enters the atmosphere, then gradually decreases because of the loss of kinetic energy. On the other hand, with the heat constraint, the dynamic pressure, given by $p_{d}=\rho V^{2} / 2$, keeps on increasing in the lower altitude region of the atmospheric trajectory despite a decrease in the velocity due to the drag. This happens because of continuously decreasing altitude leading to increasing density. On the other hand, the expression for the heating rate contains the square root of the density $\rho$ and cube of the planetocentric velocity $V$ [see Eq.(5.1)]. Thus the heating rate depends mainly on $V$ and not so much on $\rho$. This is the reason why continuously increasing density in the lower altitude region of the atmospheric trajectory with a slight decrease in $V$ does not affect the heating rate significantly. Hence, we get a nearly constant heating rate.

In Fig.5.3 the variation of g-load with $\theta$ shows that in the presence of the heat constraint, $g$-load is a little bit higher in the beginning than that in the absence of heat constraint. This is because the point where Eqs.(5.2) are satisfied, the planetocentric speed of the spacecraft is higher than that for the case when $\lambda=-1$, as some additional planetocentric velocity is lost due to penetrating further into the atmosphere (see the altitude plot in Fig. 5.1). 

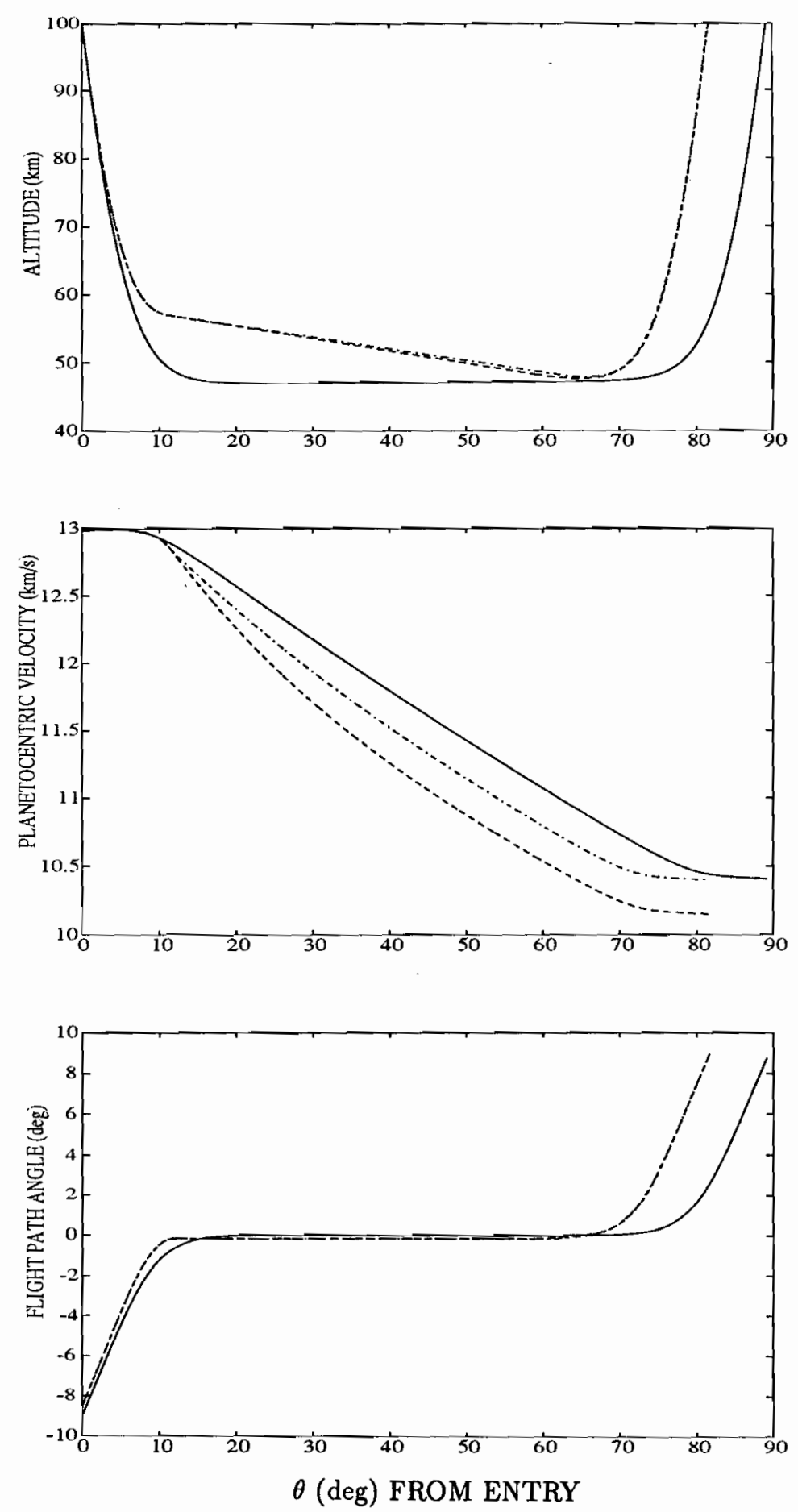

Figure 5.1: Variation of the state variables with $\theta$; - without heat constraint; - with lieat constraint, $n=2 ;-.-$ with heat constraint, $n=1.5$. 

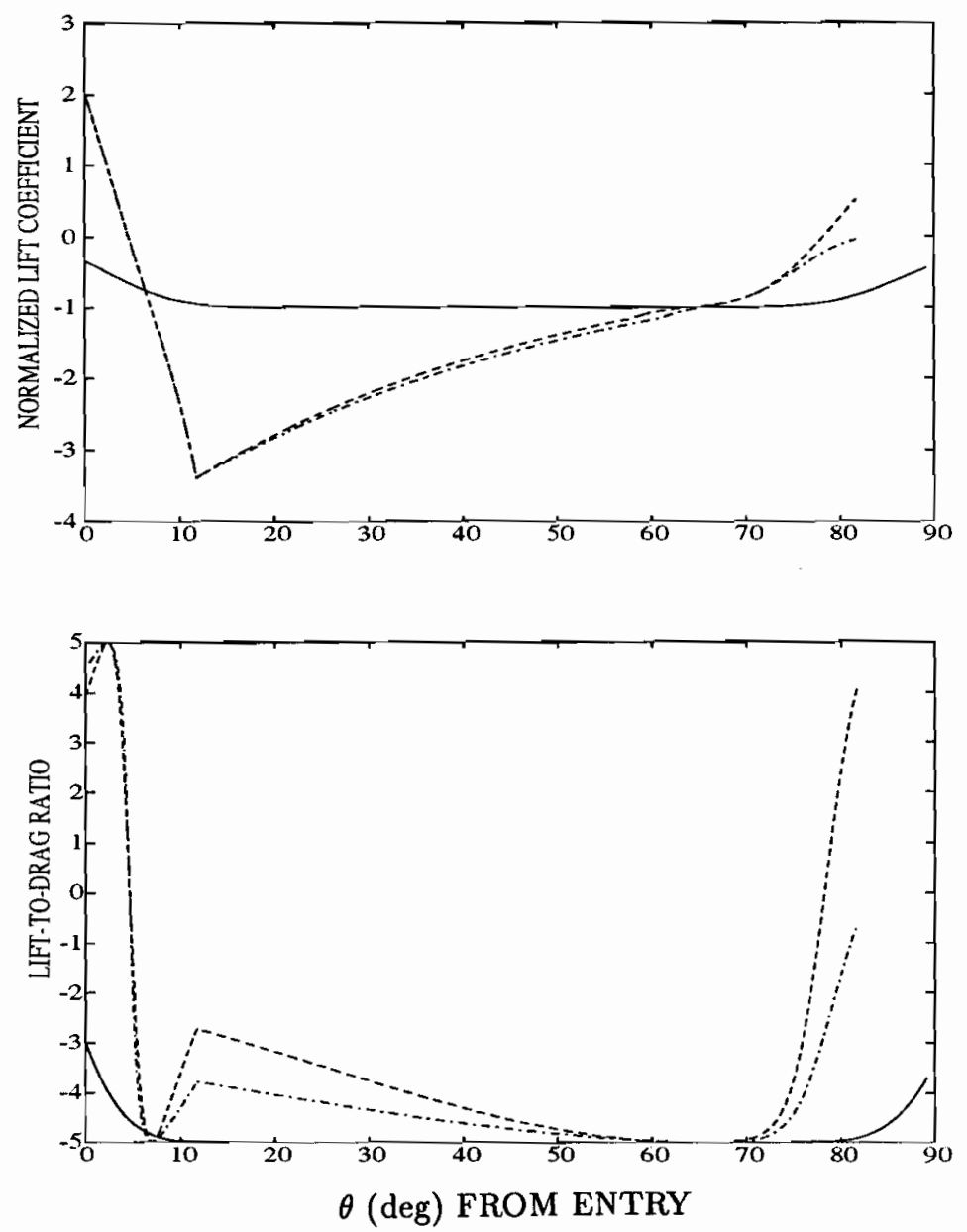

Figure 5.2: Variation of the normalized lift coefficient $\lambda$ and lift-to-drag ratio $L / D$ with $\theta$; - without heat constraint; - - with heat constraint, $n=2 ;-$. - with heat constraint, $n=1.5$. 

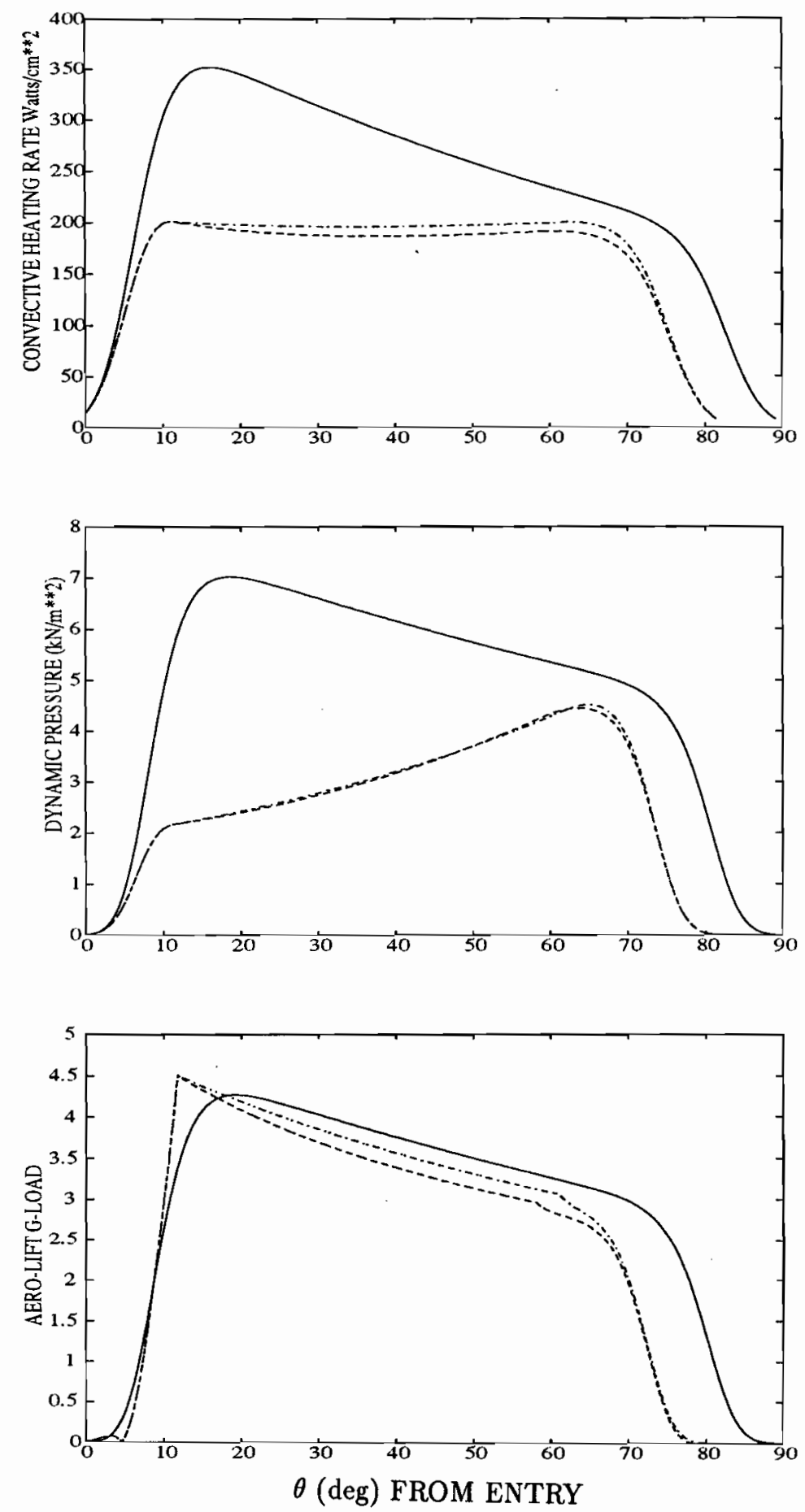

Figure 5.3: Variation of convective heating rate $q_{c}$, dynamic pressure $p_{d}$ and aero-lift g-load with $\theta$; - without heat constraint; - - with heat constraint, $n=2$; - . - with heat constraint, $n=1.5$. 


\subsubsection{Comparison of $V_{s}^{+}$without and with Heat Constraint}

Table 5.1 shows that $V_{*}^{+}$for AGA with heat constraint and $n=1.5$ is somewhat lower than that without a heat constraint. $V_{s}^{+}$for AGA with heat constraint and $n=2$, is reduced even further. A larger $V_{0}^{+}$with $n=1.5$ compared to $n=2$ (with heat constraint) is related to the former's superior overall $L / D$ ratio in the atmospheric trajectory. It was observed that in the absence of heat constraint, $V_{s}^{+}$is more or less the same whether $n$ is assumed to be 2 or 1.5. Furthermore, it can also be observed in Table 5.1 that for all the cases, the difference in $V_{a}^{+}$, for $E^{*}=5$ and $E^{*}=7$, is not negligible, rather it is substantial. This shows the significance of $E^{*}$ for any AGA mission. Hence, using $n=1.5$ in the drag polar, which is more realistic for high hypersonic speed, gives better overall $L / D$ i.e., less aerodynamic drag.

It can also be noticed from Table 5.1 that with the heat constraint case not only $q_{c_{\max }}$ is significantly reduced (for $r_{n}=1 \mathrm{~m}, q_{c_{\max }}=200 \mathrm{~W} / \mathrm{cm}^{2}$ instead of $351 \mathrm{~W} / \mathrm{cm}^{2}$ ) but $q_{r_{\max }}$ is also reduced even more significantly, i.e., from 356 to $68 \mathrm{~W} / \mathrm{cm}^{2}$. This shows that our initial judgement of Section 5.1.1 is correct, that is, when the flight takes place at higher altitudes, then if the convective heating rate is controlled, the radiative heating rate and integrated heat will be controlled automatically.

One can also notice from Table 5.1 that $\gamma_{e}$ is smaller in magnitude for AGA with the heat constraint compared to that of AGA without heat constraint. This is because with the heat constraint, a lower heating rate is required that forces the spacecraft to have a higher periapsis; this means a lower flight path angle $\left|\gamma_{e}\right|$ at the atmospheric entry. Table 5.1 also shows that $\gamma_{c}$ (i.e., the value of the flight path angle in the second regime of the atmospheric maneuvering where $q_{c}$ is constant) for the $n=1.5$ case is a little bit smaller than that for the $n=2$ case, because the overall $L / D$ for $n=1.5$ is superior to that for $n=2$ as mentioned above, which implies less drag. Thus the lower magnitude of $\gamma_{c}$ for $n=1.5$ reduces the rate of decrease of altitude (i.e., the rate of increase of density) to maintain the constant heating rate. 
Table 5.1: Variation of AGA Results with $E^{*}$

\begin{tabular}{|c|c|c|c|c|c|c|}
\hline \multirow[t]{2}{*}{ quantities } & \multicolumn{3}{|c|}{$E^{*}=5$} & \multicolumn{3}{|c|}{$E^{*}=7$} \\
\hline & $\mathrm{w} / \mathrm{o} \mathrm{hc}$ & whe $n=2$ & whe $n=1.5$ & w/o hc & whe $n=2$ & whe $n=1.5$ \\
\hline$V_{\infty}^{+}(\mathrm{km} / \mathrm{s})$ & 9.15 & 8.85 & 9.14 & 9.72 & 9.37 & 9.69 \\
\hline$V_{s}^{-}(\mathrm{km} / \mathrm{s})$ & 26.84 & 26.84 & 26.84 & 26.84 & 26.84 & 26.84 \\
\hline$V_{s}^{+}(\mathrm{km} / \mathrm{s})$ & 33.2 & 32.72 & 33.00 & 33.72 & 33.38 & 33.66 \\
\hline$\theta_{f}(\mathrm{deg})$. & 89.15 & 81.47 & 81.36 & 99.21 & 86.58 & 86.34 \\
\hline$\beta_{s}^{-}(\operatorname{deg})$. & 26.62 & 26.62 & 26.62 & 26.62 & 26.62 & 26.62 \\
\hline$\beta_{s}^{+}(\operatorname{deg})$. & 2.53 & 4.37 & 4.58 & 2.23 & 3.21 & 3.50 \\
\hline$p_{d_{\max }}\left(\mathrm{kN} / \mathrm{m}^{2}\right)$ & 7.05 & 4.43 & 4.50 & 7.07 & 4.50 & 4.61 \\
\hline $\operatorname{Max} g^{\prime} \cdot s$ & 4.25 & 4.50 & 4.50 & 4.30 & 4.53 & 4.53 \\
\hline$r_{a_{s}}\left(10^{9} \mathrm{~km}\right)$ & 7.48 & 4.94 & 6.18 & 22.22 & 9.21 & 14.21 \\
\hline$\gamma_{c}$ (deg.) & $\mathrm{N} / \mathrm{A}$ & -0.175 & -0.165 & $\mathrm{~N} / \mathrm{A}$ & -0.130 & -0.121 \\
\hline$\gamma_{\epsilon}$ (deg.) & -9.00 & -8.50 & -8.50 & -9.00 & -8.50 & -8.50 \\
\hline for $r_{n}=1 \mathrm{~m}$ & & & & & & \\
\hline$q_{c_{\text {max }}}\left(\mathrm{W} / \mathrm{cm}^{2}\right)$ & 351 & 200 & 200 & 362 & 200 & 200 \\
\hline$\psi_{r_{m a x}}\left(\mathrm{~W} / \mathrm{cm}^{2}\right)$ & 356 & 68 & 69 & 365 & 69 & 71 \\
\hline for $r_{n}=0.5 \mathrm{~m}$ & & & & & & \\
\hline$q_{c_{\text {max }}}\left(\mathrm{W} / \mathrm{cm}^{2}\right)$ & 497 & 283 & 283 & 512 & 283 & 283 \\
\hline$q_{r_{\text {max }}}\left(\mathrm{W} / \mathrm{cm}^{2}\right)$ & 245 & 47 & 47 & 251 & 47 & 49 \\
\hline
\end{tabular}

Note: $\mathrm{w} / \mathrm{o} \mathrm{hc}=\mathrm{AGA}$ without heat constraint, whc $=\mathrm{AG}$ A with heat constraint. 


\section{Comparison of AOT and AGA Missions}

Since AOT (aero-assisted orbit transfer) is already going through an experimental flight stage, it may be interesting to compare some important quantities related to the atmospheric trajectories for AOT and AGA. It will give us an idea as to how much an $\mathrm{AGA}$ mission is practical in view of the present technology. For comparison purposes, we have chosen an important study on atmospheric trajectory with heating rate constraint for AOT conducted by Mease and Vinh (the same study referred to in Section 4.4, but here we will discuss another part of the study which considers a heating rate constraint). For $\mathrm{AGA}$ with heating rate constraint, we have taken the results given in Table 5.1 when $V_{\infty}^{-}=12 \mathrm{~km} / \mathrm{s}$ with peak heating rate as $200 \mathrm{~W} / \mathrm{cm}^{2}$. One can see in Table 5.2 that the maximum dynamic pressure $p_{d_{\max }}$ associated with AGA with the heat constraint is only $4.5 \mathrm{kN} / \mathrm{m}^{2}$, which is about half of that for the AOT mission. The maximum aero-lift g-load for the AOT is 3.7, a little lower than that for AGA (see Table 5.2). Though the heating rate constraint imposed on AOT is $150 \mathrm{~W} / \mathrm{cm}^{2}$ which is less than that imposed for the AGA considered, the time $t_{f}$ required for atmospheric maneuvering of the spacecraft in the case of AOT is substantially higher (about 3 times higher) than that for AGA; thus the integrated heat load will be lower for AGA. This implies that an AGA mission for $V_{\infty}^{-}=12 \mathrm{~km} / \mathrm{s}$ is more or less is possible if a comparable AOT mission is flight-demonstrated.

\subsection{Sensitivity Study of Atmspheric Maneuver- ing with Density Uncertainty}

Up to now we have assumed that we know exactly the density at every altitude during the atomspheric flight of the spacecraft. But in reality, there will be some unpredictable fluctuations in the density, as mentioned in Section 2.5.1. Therefore, for real missions, a guidance algorithm capable of compensating for large unpredictable fluctuations in the atmospheric density will be required. The scope of the present study is restricted to the optimization of the atmospheric trajectory and discussion 
Table 5.2: Comparison of some important quantities for AOT and AGA.

\begin{tabular}{|l|r|r|}
\hline quantities & for $A O T$ & for $A G A$ \\
\hline$V_{e}(\mathrm{~km} / \mathrm{s})$ & 10.32 & 12.98 \\
$\gamma_{e}(\mathrm{deg})$ & -6.4 & -9.0 \\
$E^{*}$ & 1.5 & 5.0 \\
$r_{n}(\mathrm{~m})$ & 1.0 & 1.0 \\
$p_{d_{\max }}\left(\mathrm{kN} / \mathrm{m}^{2}\right)$ & 8.8 & 4.5 \\
$q_{c_{\max }}\left(\mathrm{W} / \mathrm{cm}^{2}\right)$ & 150 & 200 \\
Max $g^{\prime} s$ & 3.7 & 4.5 \\
$t_{f}(\operatorname{minutes})$ & 24.4 & 8.1 \\
\hline
\end{tabular}

of the guidance model is not part of the goal of this investigation. Nevertheless, we will discuss here briefly the effect of these fluctuations on the optimal $V_{s}^{+}$.

Lee and Grantham (1989) were the first to address the guidance problem of changing the HEO (High Earth Orbit) of OTV to LEO by using aerodynamic maneuvering while the density is fluctuating. They selected a problem from the same study by Mease and Vinh (1985), which we have chosen for the validation of our necessary optimal conditions and computer programs in Chapter 4, and showed that Pontryagin's maximum principle fails to give solution of this problem when fluctuation in the density model based on the atmospheric data derived from the STS-6 space shuttle flight (which involves $\pm 40 \%$ density fluctuation) is imposed and a feedback control is needed to handle such a problem. They solved the problem by using a Lyapunov optimal function minimizing the feedback control algorithm successfully.

Unfortunately, the problem of Mease and Vinh is much simpler then ours. In their problem, there are only two conditions to be satisfied at the exit point, as we have mentioned in Section 4.5, and they are related to two different state variables. In our case, we have to satisfy three conditions at the exit point and only one is related to a single state variable while the other two are nonlinear functions involving all the three state variables, the independent variable and lift control. Hence the algorithm 
of Lee and Grantham can not solve the present problem.

Since the main portion of the atmospheric trajectory where lift force is significant is the lower altitude region (see Fig. 5.3), therefore we have used the same approach as the one that was used for the heat constraint case to study the effect of the density fluctuations on $V_{s}^{+}$. Here in the first part $\lambda_{e}$ and $C_{e}$ will be chosen such that

$$
\frac{d \gamma}{d \theta}=0.0, \gamma_{c}=0 \text { and } \lambda=-1,
$$

which means that the altitude is constant in the lower altitude region. Furthermore, by using a set of random numbers, the fluctuation is imposed on the density model along the constant altitude boundary. The density model with fluctuations is given by

$$
\rho_{n}=\left(m_{n}+p_{n} R_{n}\right) \rho,
$$

where $\rho_{n}$ is the density after imposing the fluctuations; $m_{n}$ is a number that defines the density without fluctuations. If it is equal to 1 and the second term representing the fluctuation is absent, then the density will be the same as the one predicted by the exponential model [see Eqs (2.34) and (4.2)]. $p_{n}$ defines the percentage of the fluctuations; when $p_{n}=0.2$, the fluctuation in the density is $20 \% ; R_{n}$ is a random number, which can be positive or negative from 1 to -1 . We may point out here that the covergence of the optimal solution was not possible with small tolerances because of the imposed random fluctuations in the density along the constant altitude boundary. Thus, the TPBVP was solved with larger tolerances i.e., $1 \times 10^{-2}$. Since we need the results for rough comparison purposes, this will suffice.

Figure 5.4 shows the variation of altitude with $\theta$ without heat constraint. Solid line shows the solution with the density calculated from the exponential model and dashed line shows the solution when the average density is $20 \%$ less than that given by the exponential model. The results show that when the density is reduced by $20 \%$, the mean altitude of the lower region is decreased from 47 to $45 \mathrm{~km}$. This reduction in the altitude is logical, because to get sufficient lift force for maneuvering at $\lambda=-1$, we need a specific value of the density and if the actual density is decreased lower than that given by the nominal density model, then the spacecraft has to go deeper 
into the atmosphere to compensate for this to maneuver at $\lambda=-1$. However, the value of $V_{s}^{+}$is more or less the same as that shown in Table 5.3.

Table 5.3 shows that when $20 \%$ fluctuation is imposed on the density and $m_{n}=1$ then $V_{s}^{+}$is approximately the same as that without the density fluctuation. Furthermore, when $m_{n}=0.8$ and $p_{n}=0.2, V_{s}^{+}$is not changed much. But for $m_{n}=0.6$ and 0.4 there is a noticeable decrease in the value of $V_{s}^{+}$. As the overall density decreases, it causes an increase in $|\lambda|$ in the lower altitude region, which results in higher aerodynamic drag. Thus $V_{f}$ is decreased as well as $V_{s}^{+}$.

Hence, we can say that the fluctuation will only affect the value of $V_{s}^{+}$if the value of $m_{n}$ is substantially decreased or increased, in which case the value of $|\lambda|$ is greater that 2.0 in the lower altitude region and the overall $L / D$ decreases significantly and causes a rise in the aerodynamic drag.

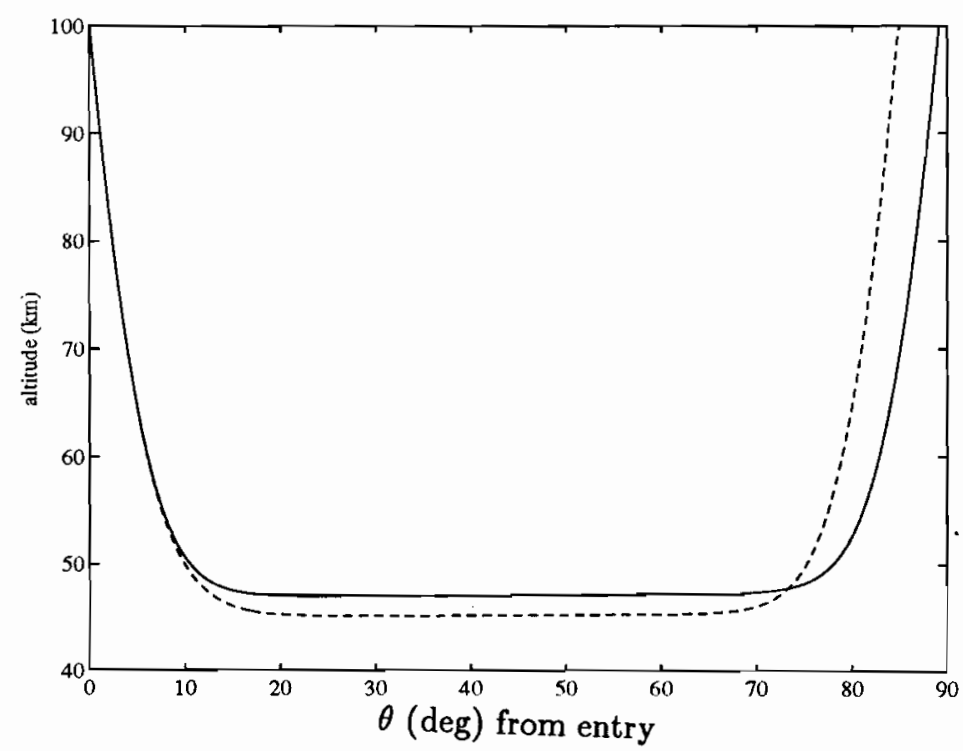

Figure 5.4: Variation of altitude with theta from entry; -- without heat constraint; - - without heat constraint with overall density $20 \%$ less than that predicted by the exponential model. 
Table 5.3: Effect of density fluctuation on $V_{s}^{+}$

\begin{tabular}{l|c|c|c|c}
\hline quantities & $\begin{array}{c}V_{\infty}^{-} \\
(\mathrm{km} / \mathrm{s})\end{array}$ & $\begin{array}{c}V_{s}^{+} \\
(\mathrm{km} / \mathrm{s})\end{array}$ & $\begin{array}{c}\theta_{f} \\
(\mathrm{deg})\end{array}$ & $\begin{array}{c}\text { lowest altitude } \\
(\mathrm{km})\end{array}$ \\
\hline w/o hc & 12.00 & 33.20 & 89.20 & 47.02 \\
w/o hc(20\% less density) & 12.00 & 33.19 & 85.02 & 45.20 \\
$\operatorname{cps}\left(m_{n}=1.0\right)$ & 12.00 & 33.20 & 82.59 & 47.02 \\
$\operatorname{cps}\left(m_{n}=0.8\right)$ & 12.00 & 33.19 & 82.59 & 47.02 \\
$\operatorname{cps}\left(m_{n}=0.6\right)$ & 12.00 & 32.93 & 74.80 & 47.02 \\
$\operatorname{cps}\left(m_{n}=0.4\right)$ & 12.00 & 32.66 & 72.92 & 47.02 \\
\hline
\end{tabular}

Note: $w / o$ hc=solution without heat constraint, $\mathrm{cps}=$ circular path in the lower altitude region when $p_{n}=0.2$. 


\section{Chapter 6}

\section{Aero-Gravity Assist for High}

\section{Energy Missions}

We have mentioned in Chapter 1 that planetary gravity assist (GA) has been used several times successfully for exploring the solar system including the recent Galileo and Ulysses missions. Ulysses has used gravity assist of Jupiter to attain $80^{\circ}$ plane change out-of-ecliptic plane for a solar polar mission. Moreover, Jupiter is the only nearest planet which can produce substantial gravity assist sufficient for any space mission. But the Earth launch energy $C_{3}\left(=V_{\infty_{E}}^{2}\right)$; required for solar flyby and remote planet missions (i.e. high energy missions) involving Jupiter gravity assist is very high, of the order of $C_{3} \approx 120 \mathrm{~km}^{2} / \mathrm{s}^{2}$ ( $C_{3}$ is the unit normally used for measuring the Earth launch energy for a space mission). Using this amount of the Earth launch energy for a space mission is not feasible for solar or remote planet mission due to the current launch constraint on $C_{3}$, which is about $81 \mathrm{~km}^{2} / \mathrm{s}^{2}$. On the other hand, utilization of multiple gravity assists is cheap fuelwise, but it may still be unable to produce a sufficient $\Delta V$ needed for high energy missions.

The main advantage of considering aero-gravity assist is its application for high energy missions. Therefore, in this chapter, aero-gravity assist for high energy missions will be discussed. Firstly, we will discuss the difficulties associated with MarsAGA for high energy missions. Then, we will consider alternative techniques for high energy missions in which AGA and GA are combined together. For example, 
by combining Mars-AGA and Jupiter-GA, not only that high heating rates can be avoided, but also the travel time and the Earth launch energy can be reduced.

In the combined scheme, the trajectory component involving Mars-AGA is obtained by using Pontryagin's maximum principle with heating rate constraint and $n=1.5$. The performance index, method of solution, the atmospheric data and the aerodynamics properties are all the same as those used in Chapter 5.

\subsection{Difficulties Associated with Mars-AGA for High Energy Missions}

Randolph and McRonald (1990) suggested $V_{\infty_{M}}^{+}=14.5 \mathrm{~km} / \mathrm{s}$ (subscript $M$ stands for the planet Mars) for MAGA for a Pluto mission; the time travel for this mission would be 6.6 years from Mars to Pluto. It was estimated by the above authors that this speed $\left(V_{\infty_{M}}^{+}\right)$would be almost within the reach of the present technology. We have now solved this problem using an optimization method with a heat constraint and Table 6.1 gives the corresponding maximum heating rates (based on the approximate models given in the previous Chapters) associated with this mission. One can note that $q_{c_{\max }}$ and $q_{r_{\max }}$ are very high (for $E^{*}=5, r_{n}=0.5 \mathrm{~m}$, they are 903 and $330 \mathrm{~W} / \mathrm{cm}^{2}$, respectively). It will not be easy to handle such heating rates for about 8 minutes without damaging the spacecraft. Table 6.1 also shows that the $V_{\infty_{B}}$ required for this mission violates the current Earth launch energy constraint, as mentioned earlier. On the other hand, heating rates associated with $V_{\infty_{M}}^{+}=20 \mathrm{~km} / \mathrm{s}$, required for a solar flyby using MAGA, will be so high that it will be difficult even to calculate them with a reasonable accuracy because of the occurrence of ablation and spalation. Therefore, it can be concluded that MAGA alone will not be sufficient for any high energy mission at this time. In the following section, some alternative techniques involving AGA will be discussed. 
Table 6.1: Different quantities related to Pluto Mission with $V_{\infty_{M}}^{+}=14.5 \mathrm{~km} / \mathrm{s}$

\begin{tabular}{lrr}
\hline relevant & \multicolumn{2}{c}{ values of $E^{*}$} \\
\cline { 2 - 3 } quantities & 5 & 7 \\
\hline \hline$V_{\infty}^{-}(\mathrm{km} / \mathrm{s})$ & 18.94 & 17.60 \\
$V_{p_{p}}{ }^{*}(\mathrm{~km} / \mathrm{s})$ & 19.57 & 18.28 \\
$V_{\infty_{E}}(\mathrm{~km} / \mathrm{s})$ & 11.05 & 10.30 \\
for $r_{n}=1 \mathrm{~m}$ & & \\
$q_{c_{\max }}\left(\right.$ Watts $\left./ \mathrm{cm}^{2}\right)$ & 634 & 516 \\
$q_{r_{\max }}\left(\right.$ Watts $\left./ \mathrm{cm}^{2}\right)$ & 480 & 313 \\
for $r_{n}=0.5 \mathrm{~m}$ & & \\
$q_{c_{\max }}\left(\right.$ Watts $\left./ \mathrm{cm}^{2}\right)$ & 903 & 729 \\
$q_{r_{\max }}\left(\right.$ Watts $\left./ \mathrm{cm}^{2}\right)$ & 330 & 215 \\
\hline$* V_{p_{p}}=$ velocity at periapsis
\end{tabular}

\subsection{Alternative: Mars-AGA-Jupiter-GA}

In this section we will discuss several alternative techniques for high energy missions. Then these techniques will be compared with respect to the travel time for the Pluto mission. It will be appropriate to first discuss briefly the method used here in calculating the travel time.

\subsubsection{Travel Time}

Kepler's third law is

$$
\tau_{p}=2 \pi\left(\frac{a^{3}}{\mu}\right)^{1 / 2}
$$

where $\tau_{p}$ is the period of an elliptic orbit, $a$ is the semimajor-axis of the orbit and $\mu$ is the universal gravitation constant multiplied by the mass of the Sun. But if we want to calculate the travel time between two points lying on an orbit separated by some angle $\theta$ or the travel time for parabolic or hyperbolic trajectories, then Eq. (6.1) is 
not useful. But by using the geometric method, we can find the travel time between two points on an elliptic, parabolic or hyperbolic trajectory (Deutsch 1963, Battin 1987).

For an elliptic orbit, one can modify Kepler's equation between two points $p_{1}$ and $p_{2}$ as

$$
t=(E-e \sin E) \frac{\tau_{p}}{2 \pi}+T
$$

where $t$ is travel time, $E$ is the eccentric anomaly given by

$$
E=2 \tan ^{-1}\left[\frac{\tan \theta / 2}{\sqrt{(1+e) /(1-e)}}\right]
$$

$\theta$ is the true anomally, $e$ is the eccentricity and $T$ is the time of pericenter passage (time measured from the pericenter to point $p_{1}$ ). If time is measured from the instant that the spacecraft is at the pericenter (i.e., $p_{1}$ is at the pericenter) then $T=0$. In other words $T$ is an integration constant that is determined by specifying the time origin.

For a hyperbolic trajectory, one can modify Kepler's equation between two points $p_{1}$ and $p_{2}$ as

$$
t=(e \sin H-H) \frac{\tau_{p}}{2 \pi}+T
$$

where $H$ is the hyperbolic anomaly and is given by

$$
H=\sinh ^{-1}\left(\frac{\sqrt{e^{2}-1} \sin \theta}{1+e \cos \theta}\right)
$$

while the semi-major axis is given by

$$
a=\frac{r(1+e \cos \theta)}{e^{2}-1} .
$$

Since the heliocentric trajectories are not known and have to be designed, we calculate the time of travel between two planets by using Eqs. (4.1). But for this purpose, we have to modify Eqs. (4.1) by replacing planetary variables with heliocentric variables, changing its independent variable from time $t$ to the radial distance between the center of the Sun and the spacecraft $r_{s}$ and removing the expressions for 


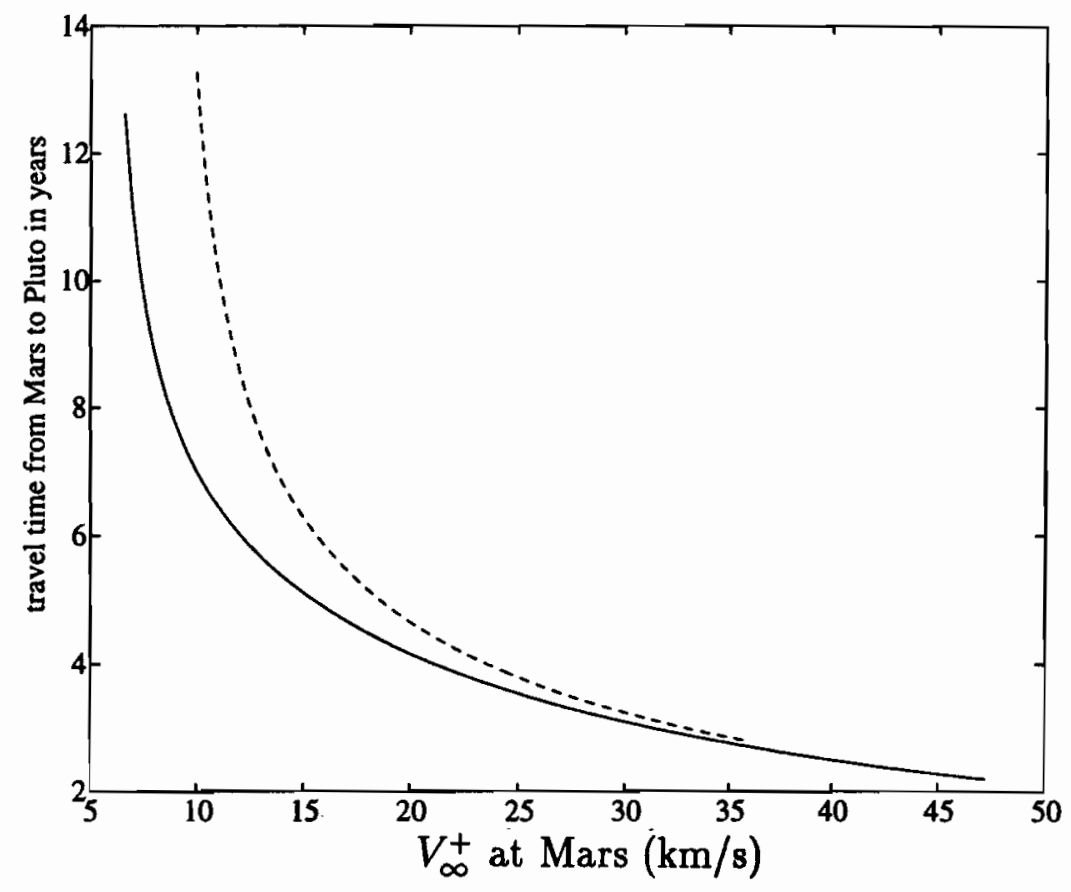

Figure 6.1- Travel time from Mars to Pluto vs $V_{\infty_{M}}^{+}$; - for MAGAJGA or VGAMAGAJGA when $r_{p}=6.0 R_{J}$ at Jupiter; - - for MAGA only, with the perihelion at Mars. 
the lift and drag forces, which will be absent in space. Hence, we have Eqs. (4.1) in modified form as

$$
\begin{aligned}
\frac{d t}{d r_{s}} & =\frac{1}{V_{s} \sin \beta_{s}} \\
\frac{d \theta_{s}}{d r_{s}} & =\frac{1}{r_{s} \tan \beta_{s}} \\
\frac{d V_{s}}{d r_{s}} & =-\frac{\mu_{s}}{r_{s}^{2} V_{s}} \\
\frac{d \beta_{s}}{d r_{s}} & =\left(\frac{1}{r_{s}}-\frac{\mu_{s}}{V_{s}^{2} r_{s}^{2}}\right) \frac{1}{\tan \beta_{s}} .
\end{aligned}
$$

We will integrate the above ordinary differential equations from the initial value of $r_{s}$ to the desired value of $r_{s}$. By this method we can calculate the travel time with good accuracy. Furthermore, above analytical equations are used to check the numerical results.

For calculating time of travel from Mars to Pluto in the case of Mars-AGA along with Jupiter-GA (MAGAJGA), first we calculate travel time from Mars to Jupiter by integrating Eqs. (6.7) with initial conditions at Mars; we assume that the perihelion of the spacecraft is at this point. Then at Jupiter, after MAGAJGA, with new initial conditions we integrate the above equations to calculate the travel time from Jupiter to Pluto. Finally, we add the travel times from Mars to Jupiter and from Jupiter to Pluto, and this will be the travel time from Mars to Pluto for MAGAJGA mission.

\subsubsection{Pluto Mission}

The disadvantages associated with using MAGA alone can be avoided by using MarsAGA-Jupiter-GA (MAGAJGA). In Fig. 6.1, a comparison of MAGAJGA and MAGA with respect to the travel time is given, which shows that MAGAJGA is considerably superior to MAGA up to $V_{\infty_{M}}^{+} \approx 15.0 \mathrm{~km} / \mathrm{s}$ in term of travel time to Pluto. The $r_{p}$ (distance of closest approach) taken in Fig. 6.1 is $6 R_{J}$ (Jupiter radius, subscript $J$ shows quantities related to the planet Jupiter) and the advantage of MAGAJGA could be even more if $r_{p}$ at Jupiter is lowered from $6 R_{J}$. Since the present technology restricts us from going up to the limit of $V_{\infty_{M}}^{+}=15 \mathrm{~km} / \mathrm{s}$, a safe $V_{\infty_{M}}^{+}$will be $10 \mathrm{~km} / \mathrm{s}$ 
Table 6.2: Comparison of JGA, MAGAJGA and VGAMAGAJGA for a Pluto mission

\begin{tabular}{|c|c|c|c|c|c|c|c|}
\hline $\begin{array}{l}\text { type of } \\
\text { mission }\end{array}$ & $\begin{array}{r}V_{\infty_{J}} \\
(\mathrm{~km} / \mathrm{s}) \\
\end{array}$ & $\begin{array}{r}V_{\infty_{M}}^{+} \\
(\mathrm{km} / \mathrm{s}) \\
\end{array}$ & $\begin{array}{r}V_{\infty_{M}}^{-} \\
(\mathrm{km} / \mathrm{s})\end{array}$ & $\begin{array}{r}V_{\infty_{E}} \\
(\mathrm{~km} / \mathrm{s})\end{array}$ & $\begin{array}{r}r_{p} \\
\left(R_{J}\right) \\
\end{array}$ & $\begin{array}{r}q_{c \max } \\
\left(W / \mathrm{cm}^{2}\right) \\
\end{array}$ & $\begin{array}{r}q_{r_{\max }} \\
\left(W / \mathrm{cm}^{2}\right)\end{array}$ \\
\hline \multicolumn{8}{|c|}{ Pluto mission, 8.5 years from Mars } \\
\hline JGA & 13.1 & N/A & N/A & 10.7 & 5.0 & N/A & $\mathrm{N} / \mathrm{A}$ \\
\hline MAGAJGA & 13.1 & 8.65 & 10.2 & 5.6 & 6.0 & 120 & 10 \\
\hline VGAMAGAJGA & 13.1 & 8.65 & 10.4 & 4.2 & 6.0 & 130 & 10 \\
\hline \multicolumn{8}{|c|}{ Pluto mission, 7 years from Mars } \\
\hline JGA & 15.7 & $\mathrm{~N} / \mathrm{A}$ & N/A & 11.7 & 5.0 & N/A & N/A \\
\hline MAGAJGA & 15.7 & 9.86 & 12.0 & 6.6 & 6.0 & 200 & 65 \\
\hline
\end{tabular}

Note: $E^{*}=7$ and $r_{n}=1 \mathrm{~m}$;

for $r_{n}=0.5 \mathrm{~m}$ the values of $q_{\mathrm{c}_{\max }}$ are 170,183 and 283 , respectively.

or less. In that velocity region MAGAJGA is significantly superior (see Fig. 6.1) so that even with $V_{\infty_{M}}^{+}=7 \mathrm{~km} / \mathrm{s}$ the travel time from Mars to Pluto is around 12 years, while in the case of simple MAGA, it is not even possible to reach Pluto with this $V_{\infty_{M}}^{+}$. One reason for lower travel time to Pluto by including Jupiter-GA, is because we can get a higher $V_{\infty}$ for a comparatively lower $V_{\infty_{M}}^{+}$, as shown in Fig. 6.2. For a given $V_{\infty_{M}}^{+}$we are able to get a $V_{\infty}$ as high as 1.6 times the former velocity. Another reason is that the heliocentric escape velocity at Jupiter is considerably lower than that at Mars. Hence, a small increase in $V_{\infty}$, can reduce the travel time to Pluto considerably.

Moreover, one can notice in Fig. 6.1 that at $V_{\infty}^{+}=10 \mathrm{~km} / \mathrm{s}$, the difference between the travel times of MAGAJGA and MAGA is very high, but this difference diminishes gradually and becomes negligible at $V_{\infty_{M}}^{+}=35 \mathrm{~km} / \mathrm{s}$. There are two reasons for this: (1) The escape velocity with respect to the Sun at Mars is 34.52 $\mathrm{km} / \mathrm{s}$. Thus, for $V_{\infty_{M}}^{+}=10.37 \mathrm{~km} / \mathrm{s}$, we have $V_{s}=$ escape velocity after AGA with respect to the Sun (i.e., parabolic trajectory), but for $V_{\infty_{M}}^{+}=11 \mathrm{~km} / \mathrm{s}$ we have a hyperbolic heliocentric trajectory after AGA with $V_{\infty_{s}}$ (heliocentric velocity very far away from the Sun) $=8.42 \mathrm{~km} / \mathrm{s}$. Furthermore, $V_{\infty}$ increases significantly with 
a relatively smaller increase in $V_{\infty_{M}}^{+}$such that for $V_{\infty_{M}}^{+}=15.00 \mathrm{~km} / \mathrm{s}$, we have a hyperbolic heliocentric trajectory after AGA with $V_{\infty_{s}}=19.81 \mathrm{~km} / \mathrm{s}$. Hence, we can see in Fig. 6.1 that above $V_{\infty}^{+}=15 \mathrm{~km} / \mathrm{s}$ the difference in travel time between MAGAJGA and MAGA is reduced considerably as at this $V_{\infty_{M}}^{+}$the values of $V_{\infty}$ 。 after MAGAJGA and MAGA are very close. (2) For MAGA we have assumed that the perihelion is at Mars, i.e., $\epsilon=0^{\circ}$. But, we have chosen $r_{p}=6.0 R_{J}$ at Jupiter for MAGAJGA. From Eqs. (2.19) and (2.20), when $V_{\infty}$, increases, $\delta$ decreases, and hence the advantage of gravity assist also decreases. Hence if $V_{\infty_{J}}$ continues to increase, then at some point $\delta$ will be so small that $\cos \epsilon=0.5$ and $V_{s}^{+}$will be much smaller than its ideal value of $\cos \epsilon=1.0$. Therefore, in Fig. 6.1, $V_{\infty_{M}}^{+}=35 \mathrm{~km} / \mathrm{s}$ is the point where due to high $V_{\infty}, V_{s}^{+}$after MAGAJGA is much smaller than that of the ideal value and the difference between the travel times of MAGAJGA and MAGA is negligible.

Table 6.2 shows that the travel time for a Pluto mission using MAGAJGA can be 7 years from Mars when $V_{\infty_{M}}^{-}=12 \mathrm{~km} / \mathrm{s}$, with substantially smaller heating rates and a $V_{\infty_{E}}$ that is considered less than what was suggested by Randolph and McRonald (64\% less in the case of MAGAJGA). If we use MAGA alone for the same $V_{\infty_{M}}^{-}=12$ $\mathrm{km} / \mathrm{s}$, then the travel time will be around 14 years.

\subsubsection{Solar Flyby Mission}

MAGAJGA can also be used effectively for solar flyby missions, with the perihelion at say 4 solar radii ( $4 R_{S}$ ) [the same perihelion is considered by McRonald and Randolph (1992) to study the Sun very closely], without having extremely high heating rates, as could be encountered in the case of solely MAGA. Table 6.3 shows that $V_{\infty_{M}}^{-}=10.2$ and $9.8 \mathrm{~km} / \mathrm{s}\left(V_{\infty_{M}}^{+}=8.65\right.$ and $\left.8.15 \mathrm{~km} / \mathrm{s}\right)$ will be required for the solar polar and the ecliptic flyby mission, respectively by using MAGAJGA, assuming that $E^{*}=7$ during maneuvering through the Martian atmosphere. It can also be noted that the associated heating rates given in Table 6.3 are substantially lower compared to that of MAGA alone (given in Table 6.1). 


\subsubsection{Venus-GA-MAGAJGA}

Venus-AGA-MAGAJGA can be very complicated. Venus-AGA is advantageous only when $V_{\infty}^{-} \geq 10 \mathrm{~km} / \mathrm{s}$, but this results in high heating rates as was shown in Chapter 4 [this was also concluded by McRonald and Randolph (1992)]. Nevertheless, Venus-Gravity-assist-MAGAJGA (or VGAMAGAJGA) could be used for high energy missions, as it will reduce $V_{\infty_{E}}$. For example, Table 6.2 shows that a travel time of 8.5 years from Mars to Pluto needs a $V_{\infty_{E}}=5.6 \mathrm{~km} / \mathrm{s}$ when MAGAJGA is used, but with VGAMAGAJGA it is reduced to $4.2 \mathrm{~km} / \mathrm{s}$ for the same travel time. Fig. 6.3 shows that the maximum value of $V_{\infty_{M}}$ that can be achieved by utilizing VGA is $V_{\infty_{E}}=4.2 \mathrm{~km} / \mathrm{s}$ and it can be sufficient for a solar flyby using MAGAJGA with $E^{*} \geq 7$. VGAMAGAJGA, of course, will impose more restrictions on launch opportunities compared to MAGAJGA.

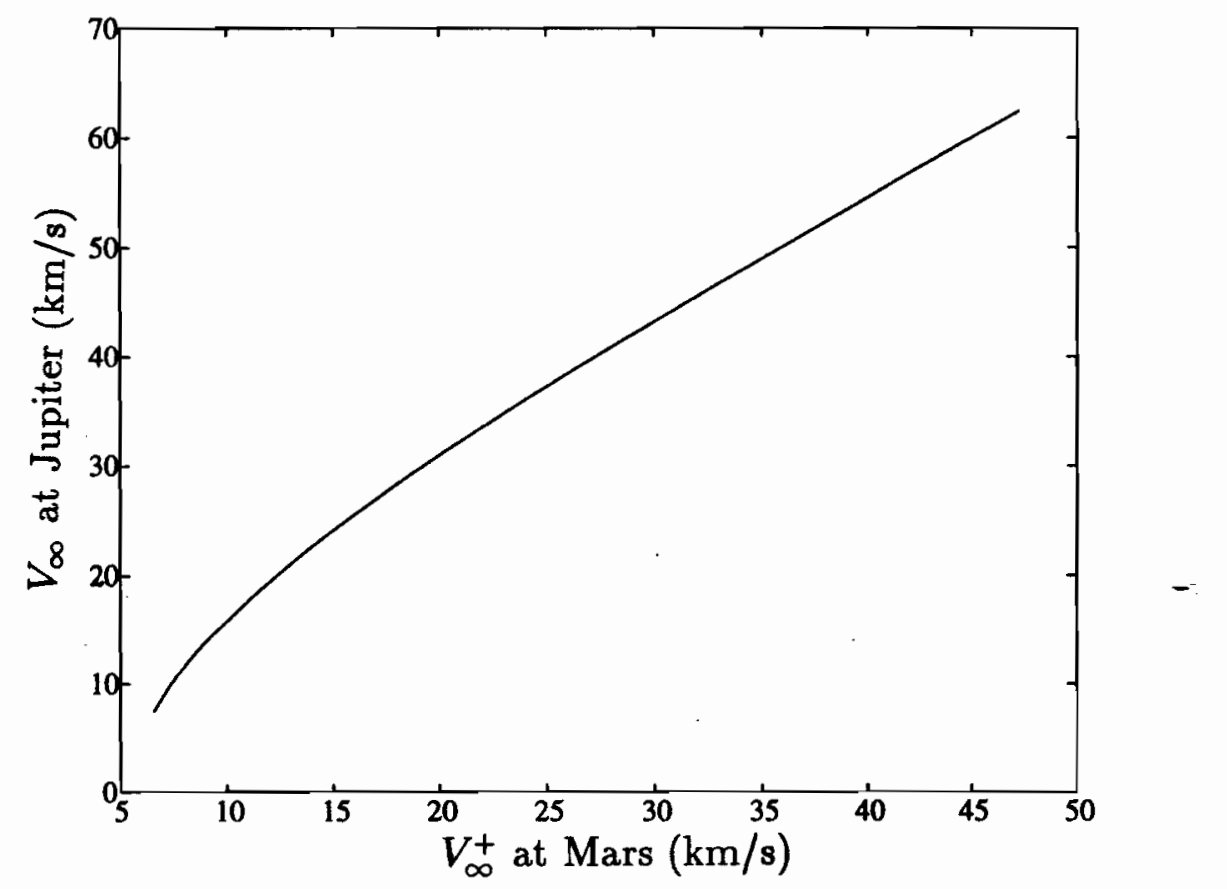

Figure 6.2: Variation of $V_{\infty_{M}}^{+}$with respect to $V_{\infty}$, after MAGA or VGAMAGA. 
Table 6.3: Potential of JGA, MAGAJGA and VGAMAGAJGA for Solar Missions

\begin{tabular}{lrrrrrrr}
$\begin{array}{l}\text { type of } \\
\text { mission }\end{array}$ & $\begin{array}{r}i_{s} \\
(\mathrm{deg})\end{array}$ & $\begin{array}{r}V_{\infty_{J}} \\
(\mathrm{~km} / \mathrm{s})\end{array}$ & $\begin{array}{r}V_{\infty_{M}}^{-} \\
(\mathrm{km} / \mathrm{s})\end{array}$ & $\begin{array}{r}V_{\infty_{E}} \\
(\mathrm{~km} / \mathrm{s})\end{array}$ & $\begin{array}{r}r_{p} \\
\left(R_{J}\right)\end{array}$ & $\begin{array}{r}q_{c_{\max }} \\
\left(W / \mathrm{cm}^{2}\right)\end{array}$ & $\begin{array}{r}q_{r_{\max }} \\
\left(W / \mathrm{cm}^{2}\right)\end{array}$ \\
\hline \hline \multirow{3}{*}{ JGA } & 0.0 & 12.0 & N/A & 10.3 & 11.1 & N/A & N/A \\
\multirow{3}{*}{ MAGAJGA } & 90 & 13.1 & N/A & 10.7 & 7.4 & N/A & N/A \\
& 0.0 & 12.0 & 9.8 & 5.5 & 8.1 & 110 & 10 \\
\multirow{4}{*}{ VGAMAGAJGA } & 90 & 13.1 & 10.2 & 5.6 & 5.8 & 120 & 6 \\
& 0.0 & 12.0 & 10.1 & 3.8 & 8.1 & 110 & 10 \\
& 90 & 13.1 & 10.4 & 4.2 & 5.8 & 130 & 6 \\
\hline
\end{tabular}

Note: $E^{*}=7, i_{s}=$ vehicle orbit inclination;

for $r_{n}=0.5 \mathrm{~m}$ the values of $q_{c_{\max }}$ in ascending order are 155,170 and 183 , respectively.

\subsection{Discussion}

MAGAJGA and VGAMAGAJGA have been discussed and compared above and it was shown that they can be alternative techniques for high energy missions with low heating rates and $V_{\infty_{E}}$. But one might raise some objections to both MAGAJGA and VGAMAGAJGA missions, which are discussed below.

- A closer flyby of Jupiter, to get large gravitational bending for achieving a higher $V_{s}$, subjects the spacecraft to considerable electron and proton bombardments, hence requiring extra shielding for the electronic parts.

The above objection is valid but needs to be examined carefully. Table 6.4 presents closest approaches of various spacecraft sent to Jupiter for gravity assist to-date from Pioneer 10 to Ulysses (Kondratyev and Hunt 1982, McLaughlin 1992). Out of these spacecraft only Pioneer 11 showed damage to its electronic parts due to intense radiation because its $r_{p}$ was $1.6 R_{J}$ (i.e., an altitude of $0.6 R_{J}$ ). In our analysis that led to Fig. $6.1, r_{p}=6 R_{J}$ was used, which is higher than the majority of the missions sent to Jupiter. Therefore the Pluto mission will not require greater shielding than 
Table 6.4: Closest approaches of spacecrafts sent for JGA

\begin{tabular}{llr}
$\begin{array}{l}\text { name of } \\
\text { spacecraft }\end{array}$ & $\begin{array}{l}\text { date of } \\
\text { launch }\end{array}$ & $\begin{array}{r}r_{p} \\
\left(R_{J^{*}}\right)\end{array}$ \\
\hline \hline Pioneer 10 & March, 1972 & 2.86 \\
Pioneer 11 & April, 1973 & 1.60 \\
Voyager 1 & September, 1977 & 4.80 \\
Voyager 2 & August, 1977 & 10.05 \\
Ulysses & October, 1990 & 6.28 \\
\hline
\end{tabular}

${ }^{*} R_{J}=71,818 \mathrm{~km}$

that used in the past. Table 6.3 shows that for the case of the solar polar flyby (perihelion=4 $R_{S}$ ) $r_{p}$ will be $5.8 R_{J}$ which is only slightly smaller than that of Pluto mission discussed above. Some additional shielding to electronic parts will prevent the damage from the radiation; this is within the grasp of present technology. On the other hand, present technology does not provide shielding to intense heating due to ablation and spalation in the case of MAGA without changing the shape of the spacecraft.

- The launch window for a Pluto mission using JGA comes every 12 years while in the case of MAGA it comes every 23 months. Hence any Pluto mission that involves JGA will restrict opportunity of launch dates.

This objection is also based on facts, but Pluto missions will not be launched very often, at least at this stage. Therefore this objection is not very serious when we know that MAGAJGA decreases heating rates, travel time and $V_{\infty_{E}}$ compared to MAGA alone. It is worth mentioning here that MAGAJGA and VGAMAGAJGA require $V_{\infty_{E}}=5.6$ and $4.2 \mathrm{~km} / \mathrm{s}$ respectively, for a solar polar flyby mission, while $V_{\infty_{E}}$ used in the case of Galileo mission (VEEGA) was only $3.7 \mathrm{~km} / \mathrm{s}$. This shows the need of MAGAJGA and VGAMAGAJGA missions; without these assists Pluto or solar missions will be difficult to realize with a small Earth launch energy. Moreover, 
the travel time from the Earth to Jupiter for these missions will be around 2.0 and 2.3 years respectively, while in the case of VEEGA mission it is 6 years.

- The final period of the solar probe in the case of MAGA will be 0.7 years whereas in the case of MAGAJGA it will be 4.5 years.

A solar flyby mission with MAGA alone is not possible at present, due to the intense heating, high $V_{\infty_{E}}$ and guidance problems. Furthermore, the Ulysses solar probe was launched with $V_{\infty_{E}}=15.4 \mathrm{~km} / \mathrm{s}\left(\Delta V_{E}=11.16 \mathrm{~km} / \mathrm{s}\right)$ with the perihelion=1.3 $\mathrm{AU}$; it used Jupiter-GA and its final period will be approximately 6 years. $V_{\infty_{E}}$ used for Ulysses mission is more than twice of that required for a MAGAJGA solar polar mission; the perihelion for the latter was chosen in this study as $4 R_{S}$, which is much lower than that of the Ulysses solar probe. Table 6.3 also shows that $V_{\infty_{E}}$ for MAGAJGA is almost half of that needed for a Jupiter-GA solar probe mission.

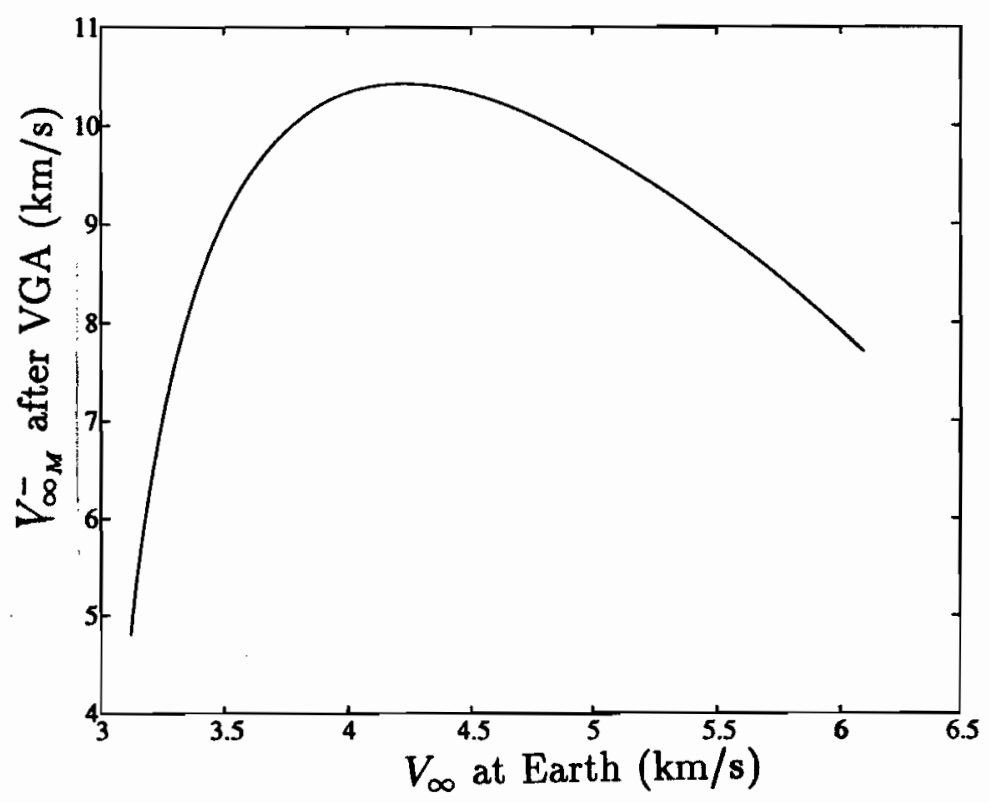

Figure 6.3: $V_{\infty}$ at Mars after VAGA with $V_{\infty_{E}}$. 


\section{Chapter 7}

\section{Optimal Aero-Gravity Assist with Heliocentric Plane Change}

Up to now, we have solved aero-gravity assist problems by maximizing the heliocentric velocity $V_{s}^{+}$, both in the presence and absence of a heating rate constraint, assuming that the orbital planes of the planets and the spacecraft are coplanar. In this chapter, we will optimize the atmospheric trajectory for AGA such that while maximizing or minimizing $V_{s}^{+}$of the spacecraft a change in the plane of the heliocentric trajectory of the spacecraft is also desired. Firstly, we will discuss the equations of motion. Then the problem is formulated variationally. Finally, the numerical results will be given for optimal aero-gravity assist with heliocentric plane change.

\subsection{Equations of Motion}

The AGA problems solved in the previous chapters show that the boundary conditions for planar atmospheric trajectory are highly nonlinear. Our experience in solving these problem also shows that it is very difficult to get convergence of the TPBVP (Two-Point Boundary Value Problem) due to the highly nonlinear boundary conditions; a lot of time is required to find the right set of initial guesses for determining the global maximum. The boundary conditions related to the heliocentric velocity $V_{s}^{+}$after three-dimensional atmospheric maneuvering will be even more 
plex than that for a planar atmospheric trajectory and the optimization problem will be a five parameter problem instead of a two parameter problem (see Section 2.6.2). Furthermore, lift force sufficient for the flight of a spacecraft with some bank angle, will be possible only in the lower altitude region, where the atmospheric density will be high enough to produce a large amount of lift. Therefore some researchers have used the constant altitude approach for solving the problem of Aero-assisted Orbit Transfer (AOT) with terrestrial plane change (Cuadra and Arthur 1966, Mease et al. 1988, Mease et al. 1991). We will also use the same constant altitude approach for solving the aero-gravity assist problem to facilitate the solution procedure. The constant altitude approach will not affect the accuracy of the results much, because a very small reduction in the planetocentric speed occurs due to the aerodynamic drag from the entrance of the atmosphere to arrival at the lower altitude region or the counterpart trajectory at the exit side. The loss in the planetocentric speed due to the drag from the atmospheric entry point to the constant altitude region will be incorporated in the problem, but we will assume that while leaving the constant altitude region to go to the atmospheric exit point, the spacecraft will use thrust to cancel drag and there will be no loss in the planetocentric speed during this period of the flight.

We can modify the equations of motion [Eqs. (2.28)-(2.33)] for the constant altitude case $\left(r=R_{r}=\right.$ constant, $\gamma=$ constant $)$ and put them in the following dimensionless form:

$$
\begin{aligned}
& \frac{d \theta}{d \tau}=\frac{v \cos \psi}{\cos \phi} \\
& \frac{d \phi}{d \tau}=v \sin \psi \\
& \frac{d v}{d \tau}=\frac{-A v^{2}}{2 E^{*}}\left\{1+\frac{\left(1-v^{2}\right)^{2}}{\cos ^{2} \sigma v^{4} A^{2}}\right\} \\
& \frac{d \psi}{d \tau}=\frac{\left(1-v^{2}\right)}{v} \tan \sigma-v \cos \psi \tan \phi
\end{aligned}
$$

The trivial equations for the constant altitude and planetocentric flight path angle are not shown. The dimensionless variables and parameters used in Eqs (7.1) are 
defined as follows:

$$
v=\frac{V}{\sqrt{R_{r} g_{p}}}, \quad \tau=\frac{t \sqrt{R_{r} g_{p}}}{R_{r}}, \quad A=\frac{\rho_{r} S R_{r} C_{L}^{*}}{2 m},
$$

where $R_{r}=$ constant altitude for aerodynamic maneuvering, $g_{p}=\mu / R_{r}^{2}$, and $\rho_{r}$ is the density at the constant altitude. Since the altitude is considered constant i.e., $d \gamma / d t=0$, we have, from Eq. (2.32),

$$
\lambda=\frac{\left(1-v^{2}\right)}{A v^{2} \cos \sigma} .
$$

\subsection{Variational Formulation}

Let $V_{s}^{+}$be the heliocentric velocity after completing the three-dimensional atmospheric maneuvering at the constant altitude $R_{r}-r_{o}$. The objective is to maximize $V_{s}^{+}$subject to the differential constraints represented by the equations of motion. Thus one can define the performance index

$$
J=V_{s}^{+}
$$

Referring to Fig 2.1, we have $V_{s}^{+}$in dimensionless form as

$$
v_{s}^{+}=\left(v_{\theta_{s}}^{2}+v_{r_{s}}^{2}\right)^{1 / 2}
$$

where

$$
v_{\theta_{s}}=v_{p}+v_{\infty}^{+} \cos \epsilon ; \quad v_{r_{s}}=v_{\infty}^{+} \sin \epsilon ; \quad v_{\infty}^{+}=\left(v_{f}^{2}-2\right)^{1 / 2} ;
$$

all the velocities are in dimensionless form i.e., dimensional velocities are divided by $\sqrt{\mu / R_{r}}$. If we want to maximize $V_{s}^{+}$then Eq. (2.60) gives the value of $\epsilon$ as

$$
\epsilon=\pi-\alpha_{t}
$$

where

$$
\alpha_{t}=\cos ^{-1}\left(\cos \theta_{t} \cos \phi_{t}\right)
$$

and

$$
\theta_{t}=\delta^{\prime}+\alpha
$$




$$
\begin{gathered}
\delta^{\prime}=\frac{\delta_{1}}{2}+\theta_{f}+\tan ^{-1}(\cos i \tan \Lambda)-\Omega, \\
\Lambda=u+\frac{\delta_{2}}{2}
\end{gathered}
$$

Since the altitude is constant therefore $\theta_{1}$ and $\theta_{2}$ are omitted. Other equations [Eqs. (2.53)-(2.61)] associated with the above equation Eqs. (7.6), given in Chapter 2, will not be repeated for the sake of brevity.

If $V_{s}^{+}$is to be minimized instead, Eqs. (7.3)-(7.5) will be modified to

$$
J=-V_{s}^{+} .
$$

Using Eqs. (2.17) and (2.18), we can rewrite $V_{s}^{+}$in dimensionless form

$$
v_{s}^{+}=\left(v_{\theta_{s}}^{2}+v_{r_{s}}^{2}\right)^{1 / 2}
$$

where

$$
v_{\theta_{s}}=v_{p}-v_{\infty}^{+} \cos \epsilon ; \quad v_{r_{s}}=v_{\infty}^{+} \sin \epsilon ; \quad v_{\infty}^{+}=\left(v_{f}^{2}-2\right)^{1 / 2}
$$

all the velocities are in dimensionless form (dimensional velocities are divided by $\sqrt{\mu / R_{r}}$. From Chapter 2, we have $\epsilon$ for the front side passage (minimizing $V_{s}^{+}$case) as

$$
\epsilon=\alpha_{t}
$$

Other relations [such as Eqs.(2.53)-(2.61)] for the maximizing $V_{s}^{+}$case will be valid here as well, except the relation for $\theta_{t}$, which is given by

$$
\theta_{t}=\alpha-\delta^{\prime}
$$

The necessary conditions for the optimal solution using Pontryagin's maximum principle are given now. First, introducing the adjoint vector $\boldsymbol{p}=\left\{p_{v}, p_{\theta}, p_{\phi}, p_{\psi}\right\}$ to form the Hamiltonian, one obtains, for $n=2$ :

$$
\begin{aligned}
\mathcal{H}= & p_{\theta} \frac{v \cos \psi}{\cos \phi}+p_{\phi} v \sin \psi+p_{v}\left[\frac{-A v^{2}}{2 E^{*}}\left\{1+\frac{\left(1-v^{2}\right)^{2}}{\cos ^{2} \sigma v^{4} A^{2}}\right\}\right] \\
& +p_{\psi}\left[\frac{\left(1-v^{2}\right)}{v} \tan \sigma-v \cos \psi \tan \phi\right]
\end{aligned}
$$


With respect to the bank angle $\sigma$, the Hamiltonian $\mathcal{H}$ can now be maximized. The first order necessary condition for an interior maximum is $\partial \mathcal{H} / \partial \sigma=0$, which leads to

$$
\tan \sigma=\frac{p_{\psi} A E^{*} v}{p_{v}\left(1-v^{2}\right)}
$$

For $\mathcal{H}$ to be maximum at a particular bank angle satisfying the above equation, we must also have

$$
\frac{\partial^{2} \mathcal{H}}{\partial \sigma^{2}}=\frac{-p_{v}\left(1-v^{2}\right)^{2}}{E^{*} A v^{2} \cos ^{2} \sigma}<0
$$

which means that always $p_{v}>0$.

The adjoints satisfy the differential equations

$$
\begin{aligned}
\frac{d p_{\theta}}{d \tau}= & 0 \\
\frac{d p_{\phi}}{d \tau}= & \frac{-p_{\theta} v \cos \psi \sin \phi}{\cos ^{2} \phi}+\frac{p_{\psi} v \cos \psi}{\cos ^{2} \phi} \\
\frac{d p_{v}}{d \tau}= & \frac{-p_{\theta} \cos \psi}{\cos \phi}-p_{\phi} \sin \psi+\frac{p_{v} A v}{E^{*}}\left[1-\frac{\left(1-v^{4}\right)}{\cos ^{2} \sigma v^{4} A^{2}}\right] \\
& +p_{\psi}\left\{\frac{\left(1+v^{2}\right) \tan \sigma}{v^{2}}+\cos \psi \tan \phi\right\} \\
\frac{d p_{\psi}}{d \tau}= & \frac{p_{\theta} v \sin \psi}{\cos \phi}-p_{\phi} v \cos \psi-p_{\psi} v \sin \psi \tan \phi .
\end{aligned}
$$

\subsubsection{Transversality Conditions}

At the initial time $\tau_{o}=0$, we have the values of the state variables

$$
\theta_{o}=\phi_{o}=\psi_{o}=0 \text { and } v_{o} \text { prescribed. }
$$

At the final time $\tau_{f}$, we have $i_{s}$ prescribed and the transversality conditions to be satisfied are

$$
\frac{p_{\phi_{f}}}{p_{\theta_{f}}}=\frac{\partial J / \partial \phi_{f}}{\partial J / \partial \theta_{f}}, p_{\psi_{f}}=\frac{\partial J}{\partial \psi_{f}}, p_{v_{f}}=\frac{\partial J}{\partial v_{f}}
$$

where

$$
\begin{aligned}
& \frac{\partial J}{\partial \theta_{f}}=\frac{v_{\infty}^{+}\left(v_{\theta_{s}} \sin \epsilon-v_{r_{s}} \cos \epsilon\right)}{v_{s}^{+}} \frac{\partial \alpha_{t}}{\partial \theta_{f}} \\
& \frac{\partial J}{\partial \phi_{f}}=\frac{v_{\infty}^{+}\left(v_{\theta_{s}} \sin \epsilon-v_{r_{s}} \cos \epsilon\right)}{v_{s}^{+}} \frac{\partial \alpha_{t}}{\partial \phi_{f}}
\end{aligned}
$$




$$
\begin{aligned}
\frac{\partial J}{\partial \psi_{f}} & =\frac{v_{\infty}^{+}\left(v_{\theta_{s}} \sin \epsilon-v_{r_{s}} \cos \epsilon\right)}{v_{s}^{+}} \frac{\partial \alpha_{t}}{\partial \psi_{f}} \\
\frac{\partial J}{\partial v_{f}} & =\frac{v_{f}\left(v_{\theta_{s}} \cos \epsilon+v_{r_{s}} \sin \epsilon\right)}{v_{s}^{+} v_{\infty}^{+}}+\frac{\partial \alpha_{t}}{\partial v_{f}} \frac{v_{\infty}^{+}\left(v_{\theta_{s}} \sin \epsilon-v_{r_{s}} \cos \epsilon\right)}{v_{s}^{+}} .
\end{aligned}
$$

In Eqs. (7.21), we have to use ratio of two of the final co-states, because of the additional condition of prescribed heliocentric plane change $i_{s}$ to be satisfied, we are left with only three transversality conditions to solve the TPBVP. Since all of the four state variables appear in the performance index, therefore all the final four co-states have be included in the transversality conditions. Thus we have arbitrarily taken the ratio of the final co-states of $\phi$ and $\theta$ so that all the co-states should be part of the transversality conditions to be satisfied. For the case of minimizing, $V_{s}^{+}$, Eqs. (7.22) become

$$
\begin{aligned}
\frac{\partial J}{\partial \theta_{f}} & =\frac{-v_{\infty}^{+}\left(v_{\theta_{s}} \sin \epsilon+v_{r_{s}} \cos \epsilon\right)}{v_{s}^{+}} \frac{\partial \alpha_{t}}{\partial \theta_{f}} \\
\frac{\partial J}{\partial \phi_{f}} & =\frac{-v_{\infty}^{+}\left(v_{\theta_{s}} \sin \epsilon+v_{r_{s}} \cos \epsilon\right)}{v_{s}^{+}} \frac{\partial \alpha_{t}}{\partial \phi_{f}} \\
\frac{\partial J}{\partial \psi_{f}} & =\frac{-v_{\infty}^{+}\left(v_{\theta_{s}} \sin \epsilon+v_{r_{s}} \cos \epsilon\right)}{v_{s}^{+}} \frac{\partial \alpha_{t}}{\partial \psi_{f}} \\
\frac{\partial J}{\partial v_{f}} & =\frac{-v_{f}\left(-v_{\theta_{s}} \cos \epsilon+v_{r_{s}} \sin \epsilon\right)}{v_{s}^{+} v_{\infty}^{+}}-\frac{\partial \alpha_{t}}{\partial v_{f}} \frac{v_{\infty}^{+}\left(v_{\theta_{s}} \sin \epsilon+v_{r_{s}} \cos \epsilon\right)}{v_{s}^{+}} .
\end{aligned}
$$

The values of $\partial \alpha_{t} / \partial \theta_{f}, \partial \alpha_{t} / \partial \phi_{f}, \partial \alpha_{t} / \partial \psi_{f}$ and $\partial \alpha_{t} / \partial v_{f}$ are quite lengthy and therefore are omitted here for brevity; for details see Appendix B.

To search for the optimal aerogravity assist, we must guess the initial $p_{\phi_{o}}, p_{\psi_{\circ}}$ and $p_{v_{o}}$ while computing $p_{\theta_{o}}$ from the Hamiltonian expression given in Eq. (7.13). Since the time is free and the Hamiltonian does not contain the independent variable, therefore from Section 3.1.1 we have $\mathcal{H}=0$.

Now there are eight differential equations, namely Eqs.(7.1) for the four states and Eqs. (7.16)-(7.19) for the four co-states. Integration of these equations will yield extremal trajectories for a number of situations depending on the initial values of the co-states. This problem is a three parameter TPBVP, and we have to guess the initial values of $p_{\phi_{0}}, p_{\psi_{o}}$ and $p_{v_{o}}$ for solving the problem. The accuracy of the numerical integration is checked by examining the Hamiltonian, which must be equal to zero. 


\subsection{Method of Solution}

The calculations were carried out with a specified value of $V_{\infty}^{-}$for the planet Mars. Different values of $V_{\infty}^{-}$imply different heliocentric trajectories form the Earth to Mars. The TPBVP can be solved using the shooting method by choosing the initial guesses as mentioned above, Eqs. (7.1), (7.16)-(7.19) are integrated from $\tau=0$ until $i_{s}$ is equal to its prescribed value. The integration is performed by Adams-Moulton method up to order twelve, while the local absolute error is controlled to be less than $1.0 \times 10^{-8}$. In all the cases studied, it has been possible, after iterations, to find a set of initial values of $p_{\phi_{o}}, p_{\psi_{o}}$ and $p_{v_{o}}$ such that the boundary conditions are satisfied at the specified $i_{s}$ value.

The trajectory and control computed are optimal since the necessary conditions (7.16)-(7.19), initial conditions (7.20) and the transversality conditions (7.21) are satisfied. There are more than one local maxima but the global maximum can be identified by examining the constant altitude atmospheric trajectory. For example, in the case of the global maximum, $\theta_{f}$ must be significantly larger, $\phi_{f}$ should be very small so that the spacecraft can get high $\phi_{t}$ at a distance far away from the planet and $i_{n}$ should be small to give higher $V_{s}^{+}$. Moreover, the spacecraft should fly with minimum aerodynamic drag during atmospheric maneuvering.

\subsection{Results and Discussion}

For all the cases considered below, the planet chosen for AGA with heliocentric plane change was Mars and $V_{\infty}^{-}$was taken as $10 \mathrm{~km} / \mathrm{s}$. The data for the heliocentric orbit transfer, the planetary orbit transfer, the planetary atmospheric density and the aerodynamic properties of the spacecraft are the same as those used in the previous chapters. The constant altitude was taken as $44 \mathrm{~km}$ for all the cases in this section.

The results are presented in three sets. In the first set, AGA results for the heliocentric plane change problem while maximizing $V_{s}^{+}$are presented. In the second set, AGA results for the heliocentric plane change problem while minimizing $V_{s}^{+}$are presented. Finally, AGA for the heliocentric plane change problem while minimizing 
$V_{s}^{+}$is examined for different angles of prescribed heliocentric plane change.

\subsubsection{Maximum $V_{s}^{+}$with Heliocentric Plane Change}

The results for optimal AGA with prescribed heliocentric plane change $i_{s}=4.5^{\circ}$, while maximizing $V_{s}^{+}$, are shown in Table 7.1 for two cases i.e., without initial heliocentric inclination and with an initial heliocentric plane inclination of $i_{s_{o}}=3.0^{\circ}$.

Table 7.1 shows that $V_{s}^{+}$for AGA with a heliocentric plane change is less than that without plane change (i.e., $30.27 \mathrm{~km} / \mathrm{s}$ opposed to $31.65 \mathrm{~km} / \mathrm{s} ; E^{*}=5$ in both cases). If a thrust impulse, $\Delta V_{t}$, is used for the same $4.5^{\circ}$ plane change after planar optimal AGA, then one will need $\Delta V_{t}=2.47 \mathrm{~km} / \mathrm{s}$. This means that AGA with heliocentric plane change is more fuel efficient and saves a $\Delta V$ of $1.09 \mathrm{~km} / \mathrm{s}(2.47$ $(31.65-30.27))$ [fuel saving $=\Delta V_{t}-V_{s}^{+}($without heliocentric plane change $)+V_{s}^{+}($with heliocentric plane change)].

From Table 7.1 one notes that $V_{s}^{+}$for $E^{*}=7$ cases are substantially higher than those for $E^{*}=5$. This shows the importance of using a higher $E^{*}$ for an AGA mission, which we have already seen in the case of planar optimal AGA. In all cases, we can see that $\phi_{f}$ is very small because Eqs. (2.57) and (7.7) imply that to get a higher $\phi_{t}$, one must have a lower $\phi_{f}$ and higher $\psi_{f}$ (as $i_{s}$ is a function of $\phi_{t}$ ). Furthermore, when $V_{s}^{+}$is being maximized, $i_{t}^{\prime}$ can not be large enough to give high $i_{s}\left(i_{s}\right.$ is a function of $\zeta$ and $i_{t}^{\prime}$ ). Because in this case, $V_{s}$ will always be significantly greater than $V_{\infty}^{+}$. Hence we can get only a small heliocentric plane change in the case of maximizing $V_{s}^{+}$. This point will be explained in more detail in the next section.

We can also see from Table 7.1 that an initial inclination $i_{s_{o}}=3^{\circ}$ does not change the values of $V_{s}^{+}$much. Thus we can conclude that a small initial heliocentric inclination will not give us much advantage in getting a higher $V_{s}^{+}$and $i_{s}$ after AGA. Higher value of $i_{s_{o}}$ will increase $\Delta V_{E}$ substantially and will increase $V_{\infty}^{-}$to some extent; this case needs further study and will not be discussed here further.

The maximum convective and radiative heating rates are also given in Table 7.1. If they are compared with the heating rate of unconstrained optimal Mars-AGA case given in Table 5.4, then we can see that although the density at the constant altitude 
Table 7.1: Maximization of $V_{s}^{+}$with $i_{s}=4.5 \mathrm{deg}$.

\begin{tabular}{|c|c|c|c|c|}
\hline & \multicolumn{2}{|c|}{$E^{*}=5$} & \multicolumn{2}{|c|}{$E^{*}=7$} \\
\hline & $i_{s_{o}}=0$ & $i_{s_{o}}=3^{\circ}$ & $i_{s_{0}}=0$ & $i_{s_{o}}=3^{\circ}$ \\
\hline$V_{\infty}^{+}(\mathrm{km} / \mathrm{s})$ & 6.49 & 6.50 & 7.45 & 7.52 \\
\hline$V_{s}^{-}(\mathrm{km} / \mathrm{s})$ & 25.32 & 25.32 & 25.32 & 25.32 \\
\hline$V_{s}^{+}(\mathrm{km} / \mathrm{s})$ & 30.27 & 30.29 & 31.26 & 31.34 \\
\hline$V_{s}^{+}\left(\right.$for $\left.i_{s}=0, \mathrm{~km} / \mathrm{s}\right)$ & 31.65 & - & 32.35 & 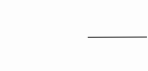 \\
\hline$\delta^{\prime}$ (deg.) & 100.02 & 100.08 & 91.86 & 91.39 \\
\hline$\phi_{f}$ (deg.) & 3.96 & 3.72 & -2.76 & -0.68 \\
\hline$\psi_{f}$ (deg.) & 23.20 & 22.75 & 20.35 & 19.43 \\
\hline$i$ (deg.) & 23.52 & 23.04 & 20.53 & 19.44 \\
\hline$\theta_{f}($ deg. $)$ & 81.36 & 81.42 & 75.50 & 75.15 \\
\hline$\epsilon$ (deg.) & 21.94 & 21.60 & 19.78 & 19.42 \\
\hline$\phi_{t}(\mathrm{deg})$. & 21.52 & 21.15 & 19.42 & 18.96 \\
\hline$i_{n}$ (deg.) & 11.54 & 5.01 & 11.23 & 5.78 \\
\hline$\zeta($ deg. $)$ & 4.60 & 4.53 & 4.62 & 4.69 \\
\hline$\phi_{t}^{\prime}($ deg. $)$ & 4.62 & 4.54 & 4.63 & 4.55 \\
\hline$i_{t}^{t}$ (deg.) & 78.46 & 78.15 & 78.77 & 77.28 \\
\hline$\beta_{s}^{-}$(deg.) & 23.25 & 23.25 & 23.25 & 23.25 \\
\hline$\beta_{s}^{+}($deg. $)$ & 0.92 & 0.39 & 0.89 & 0.45 \\
\hline for $r_{n}=0.5 \mathrm{~m}$ & & & & \\
\hline$q_{c_{\max }}\left(\mathrm{W} / \mathrm{cm}^{2}\right)$ & 393 & 393 & 393 & 393 \\
\hline$q_{r_{\max }}\left(\mathrm{W} / \mathrm{cm}^{2}\right)$ & 78 & 78 & 78 & 78 \\
\hline for $r_{n}=1 \mathrm{~m}$ & & & & \\
\hline$q_{c_{\max }}\left(\mathrm{W} / \mathrm{cm}^{2}\right)$ & 278 & 278 & 278 & 278 \\
\hline$q_{r_{\max }}\left(\mathrm{W} / \mathrm{cm}^{2}\right)$ & 114 & 114 & 114 & 114 \\
\hline
\end{tabular}


region in the present case is significantly higher than that of Table 5.4 for Mars-AGA (where the constant altitude was approximately $47 \mathrm{~km}$ as opposed to $44 \mathrm{~km}$ here), the heating rate is higher by only $55 \mathrm{~W} / \mathrm{cm}^{2}$. The calculations were carried out with $r_{n}=1 \mathrm{~m}$. The heating rate could be decreased if we increase the constant altitude and use the $n=1.5$ drag polar i.e., to fly at around $\left|\lambda_{\text {max }}\right|$.

\subsubsection{Minimum $V_{s}^{+}$with Heliocentric Plane Change}

The results for optimal aero-gravity assist with a prescribed heliocentric plane change of $6^{\circ}$, while minimizing $V_{s}^{+}$, are shown in Table 7.2 for two cases, without and with initial heliocentric inclination $i_{s_{o}}=3^{\circ}$, for two values of $E^{*}, 5$ and 7 .

Table 7.2 shows that $V_{s}^{+}$with heliocentric plane change $i_{s}=6^{\circ}$ is less than that without heliocentric plane change. If a thrust impulse, $\Delta V_{t}$, is used to realize the heliocentric plane change after planar AGA for the same $i_{s}=6^{\circ}$, then we need $\Delta V_{t}=1.74 \mathrm{~km} / \mathrm{s}$, for $E^{*}=5$. This means AGA with heliocentric plane change is 1.13 $\mathrm{km} / \mathrm{s}$ more (1.74-(17.25-16.64)) fuel efficient.

Similar to Table 7.1, Table 7.2 also shows that the minimized $V_{s}^{+}$for $E^{*}=7$ is noticeably lower than that for $E^{*}=5$. Hence AGA mission with heliocentric plane change would be considerably more fuel efficient for $E^{*}=7$. We can notice by comparing Tables 7.1 and 7.2 that $i_{s}$ used for the minimum $V_{s}^{+}$case is not only higher but $V_{\infty}^{+}$for it is also larger than that of the maximum $V_{s}^{+}$case. This is because the value of latitude at far away distance, $\phi_{t}^{\prime}$, is higher for the minimum $V_{s}^{+}$case, where $\phi_{t}^{\prime}$ is the function of the ratio of $V_{\infty}^{+}$and $V_{s}^{+}$respectively. In the case of minimization of $V_{s}^{+}$, the ratio is greater than that for the maximization of $V_{s}^{+}$, and as a result of that we find that the average value of $\phi_{t}^{\prime}$ for the minimization case is 2 deg. higher than that for the maximization case. Thus the heliocentric plane change will always be higher for the minimization case than the maximization case (provided $V_{\infty}^{-}$is the same). We can notice from Tables 7.1 and 7.2 that $i_{n}\left(\phi_{t}^{\prime}, \zeta\right)$ is always small, because $\phi_{t}^{\prime}$ is function of $V_{\infty}^{+}, V_{s}^{+}$and $\phi_{t}$, a small value of $i_{n}$ is only possible when the ratio of $\sin \phi_{t}^{\prime}$ and $\sin \zeta$ is nearly equal to 1 . Since in the case of $V_{s}^{+}$maximization, $\phi_{t}^{\prime}$ is smaller and to get $\sin \phi_{t}^{\prime} / \sin \zeta \approx 1$ we need a small $V_{r s}$, which is only possible with a 
Table 7.2: Minimization of $V_{s}^{+}$with $i_{s}=6.0 \mathrm{deg}$.

\begin{tabular}{|c|c|c|c|c|}
\hline & \multicolumn{2}{|c|}{$E^{*}=5$} & \multicolumn{2}{|c|}{$E^{*}=7$} \\
\hline & $i_{s_{o}}=0$ & $i_{s_{o}}=3^{\circ}$ & $i_{s_{o}}=0$ & $i_{s_{o}}=3^{\circ}$ \\
\hline$V_{\infty}^{+}(\mathrm{km} / \mathrm{s})$ & 7.24 & 7.25 & 7.89 & 7.90 \\
\hline$V_{s}^{-}(\mathrm{km} / \mathrm{s})$ & 25.32 & 25.32 & 25.32 & 25.32 \\
\hline$V_{s}^{+}(\mathrm{km} / \mathrm{s})$ & 17.25 & 17.21 & 16.55 & 16.53 \\
\hline$V_{s}^{+}\left(i_{s}=0, \mathrm{~km} / \mathrm{s}\right)$ & 16.64 & - & 15.95 & - \\
\hline$\delta^{\prime}$ (deg.) & 81.53 & 81.32 & 82.23 & 82.01 \\
\hline$\phi_{f}$ (deg.) & 4.74 & 3.91 & 3.20 & 3.49 \\
\hline$\psi_{f}$ (deg.) & 15.7 & 13.42 & 13.57 & 13.26 \\
\hline$i$ (deg.) & 16.45 & 13.71 & 14.03 & 13.71 \\
\hline$\theta_{f}$ (deg.) & 63.92 & 63.74 & 66.00 & 65.77 \\
\hline$\epsilon$ (deg.) & 14.83 & 14.51 & 12.98 & 12.75 \\
\hline$\phi_{t}$ (deg.) & 14.47 & 12.45 & 12.72 & 12.45 \\
\hline$i_{n}(\mathrm{deg})$. & 13.39 & 7.41 & 12.23 & 6.35 \\
\hline$\zeta$ (deg.) & 6.17 & 6.03 & 6.14 & 6.05 \\
\hline$\phi_{t}^{\prime}$ (deg.) & 6.02 & 5.21 & 6.15 & 5.95 \\
\hline$i_{t}^{\prime}$ (deg.) & 76.61 & 75.64 & 77.77 & 79.58 \\
\hline$\beta_{s}^{-}$(deg.) & 23.25 & 23.25 & 23.25 & 23.25 \\
\hline$\beta_{s}^{+}$(deg. $)$ & 1.42 & 0.78 & 1.31 & 0.00 \\
\hline for $r_{n}=0.5 \mathrm{~m}$ & & & & \\
\hline$q_{c_{\max }}\left(\mathrm{W} / \mathrm{cm}^{2}\right)$ & 393 & 393 & 393 & 393 \\
\hline $\begin{array}{l}q_{r_{\max }}\left(\mathrm{W} / \mathrm{cm}^{2}\right) \\
\text { for } r_{n}=1 \mathrm{~m}\end{array}$ & 78 & 78 & 78 & 78 \\
\hline$q_{c_{\max }}\left(\mathrm{W} / \mathrm{cm}^{2}\right)$ & 278 & 278 & 278 & 278 \\
\hline$q_{r_{\max }}\left(\mathrm{W} / \mathrm{cm}^{2}\right)$ & 114 & 114 & 114 & 114 \\
\hline
\end{tabular}


higher $\theta_{f}$ when $\sin \epsilon \approx 0$. Therefore we can see that $\theta_{f}$ for the maximization case is higher than that for minimization, while $V_{\infty}^{+}$is substantially smaller due to the drag. Furthermore, in all cases $i_{n}$ is small, which is useful when the prescribed $i_{s}$ is higher. But in the cases when $i_{s}$ is $6^{\circ}$ or less, then a constraint on $i_{n}$ of $25^{\circ}$ to $35^{\circ}$ can give us a slightly smaller minimum $V_{s}^{+}$and a substantially higher maximum $V_{s}^{+}$(specially for $E^{*}=5$ when higher $\theta_{f}$ reduces $V_{\infty}^{+}$considerably).

We can also observe from Tables 7.1 and 7.2 that the final latitude, $\phi_{f}$, is higher for $E^{*}=5$ than for $E^{*}=7$. This happens because to increase or reduce $V_{s}^{+}, \theta_{f}$ must be larger for $E^{*}=7$ (since in that case the drag will be lower and a higher $\theta_{f}$ will decrease $\epsilon$ without reducing $V_{\infty}^{+}$much); after that if $\phi_{f}$ is kept the same as for $E^{*}=5$, then $\phi_{t}$ will be smaller due to larger $\theta_{f}$ [see Eqs. (2.57), (7.7) and (2.59)]. Thus to maintain the value of $\phi_{t}$ closer to that for the $E^{*}=5$ cases, $\phi_{f}$ must be lower for the $E^{*}=7$ cases to give the same $i_{s}$ and a greater change in $V_{s}^{+}$.

\subsubsection{Minimization of $V_{s}^{+}$with Different Angles of Helio- centric Plane Change}

The results for optimal AGA with different prescribed angles of heliocentric plane change i.e., $6^{\circ}, 9^{\circ}$ and $12^{\circ}$, while minimizing $V_{s}^{+}$, are shown in Table 7.3 for $E^{*}=7$. One can note that as $i_{s}$ increases, the fuel saving increases as well. $V_{\infty}^{+}$and $\theta_{f}$ for all the cases are nearly equal, but $V_{s}^{+}$increases as $i_{s}$ increases; because for higher $i_{s}$, we need to have higher $\phi_{t}$ or $i$ which causes $\delta^{\prime}$ and $\alpha_{t}$ to decrease and eventually we get a higher $V_{s}^{+}$[see Eqs. (7.6), (7.7) and (2.61)]. Hence $V_{s}^{+}$increases to $17.85 \mathrm{~km} / \mathrm{s}$ for $i_{s}=12^{\circ}$, compared with 16.55 and $16.99 \mathrm{~km} / \mathrm{s}$ for $i_{s}=6^{\circ}$ and $9^{\circ}$, respectively.

Table 7.3 also reveals that final latitude, $\phi_{f}$, increases as $i_{s}$ decreases, because to have a higher $\phi_{t}$ we must increase final heading angle, $\psi_{f}$, so that $i$ can be high when $\phi_{f}$ is low and a higher $\psi_{f}$ automatically gives rise to $\phi_{f}$ as $d \phi / d t(\psi, V)$. Furthermore, if we want to change the heliocentric plane of the spacecraft by $12^{\circ}$ then the fuel saving is $1.45 \mathrm{~km} / \mathrm{s}$ compared to planar AGA combined with a thrust impulse, which is a substantial amount of fuel saving for a space mission. Though we have used a fairly small $V_{\infty}^{-}(10 \mathrm{~km} / \mathrm{s})$ to avoid high heating rates, it could give us a heliocentric plane 
change up to $12^{\circ}$ in addition to a substantial decrease in $V_{s}^{+}$(i.e., $7.43 \mathrm{~km} / \mathrm{s}$ ) and there is a $1.45 \mathrm{~km} / \mathrm{s}$ fuel saving.

Figures 7.1 and 7.2 show the variation of $\sigma, \lambda$ and $\phi$ with time for $i_{s}=6^{\circ}$ and $12^{\circ}$, respectively. Figure 7.1 shows that in the beginning, the normalized lift coefficient $|\lambda|$ decreases (it is clearer for $i_{s}=6^{\circ}$ case), then it increases while the absolute value of bank angle $|\sigma|$ continuously increases. For the $i_{s}=12^{\circ}$ case, the only reason for the slight decrease in $|\lambda|$ is that, as long as $|\sigma|$ is very small $|\lambda|$ decreases due to the aerodynamic drag, which constantly reduces the planetocentric speed $V$. But in the case of $i_{s}=6^{\circ},|\lambda|$ decreases further because of decreasing $|\sigma|$, which makes it more noticeable.

We can see from Fig. 7.1 that for $i_{s}=6^{\circ}$, the value of $\sigma$ is positive in the beginning but later it becomes negative; during this period the spacecraft attains a slight negative latitude $\phi$ and heading angle $\psi$, then they become positive due to the negative bank angle $\sigma$. In this way we get more bending angle $\theta_{f}$ without getting higher $\phi_{f}$ and $\psi_{f}$ as the rate of decrease of $\sigma$ is nearly constant. For the case of $i_{s}=12^{\circ}$, the rate of increase $|\sigma|$ is higher (initially $|\sigma|$ is around zero and then increases), since we need a high $\phi_{t}$ for a larger value of $i_{s}$. The high value of $\phi_{t}$ is only possible with higher $i\left(\phi_{f}, \psi_{f}\right)$ [provided $\Lambda=u+\delta_{2} / 2-\theta_{2}$ is small, see Eq. (2.57)], as shown in Table 7.3.

For all the other cases considered earlier, the variations of $\phi, \psi, \sigma$ and $\lambda$ are similar to those given in Figs. 7.1 and 7.2. It was noted that a reduction in the computational tolerance does not affect the value of $V_{s}^{+}$, but it causes a slight decrease in the initial value of $\sigma$. 
Table 7.3: Minimum $V_{s}^{+}$with different $i_{s}$

\begin{tabular}{|c|c|c|c|}
\hline & \multicolumn{3}{|c|}{$i_{s}$ (deg.) } \\
\hline & 6.00 & 9.00 & 12.00 \\
\hline$V_{\infty}^{+}(\mathrm{km} / \mathrm{s})$ & 7.89 & 7.93 & 7.81 \\
\hline$V_{s}^{-}(\mathrm{km} / \mathrm{s})$ & 25.32 & 25.32 & 25.32 \\
\hline$V_{s}^{+}(\mathrm{km} / \mathrm{s})$ & 16.55 & 16.99 & 17.85 \\
\hline$V_{s}^{+}\left(i_{s}=0, \mathrm{~km} / \mathrm{s}\right)$ & 15.95 & 15.95 & 15.95 \\
\hline$\Delta V_{t}(\mathrm{~km} / \mathrm{s})$ & 1.67 & 2.51 & 3.35 \\
\hline fuel saving $(\mathrm{km} / \mathrm{s})$ & 1.07 & 1.47 & 1.45 \\
\hline$\delta^{\prime}$ (deg.) & 80.23 & 76.90 & 74.32 \\
\hline$\phi_{f}$ (deg.) & 3.62 & 8.14 & 11.85 \\
\hline$\psi_{f}$ (deg.) & 13.57 & 21.67 & 32.29 \\
\hline$i$ (deg.) & 14.03 & 23.08 & 34.17 \\
\hline$\theta_{f}(\mathrm{deg})$. & 66.00 & 61.03 & 58.86 \\
\hline$\epsilon($ deg. $)$ & 12.98 & 21.65 & 30.41 \\
\hline$\phi_{t}$ (deg.) & 12.72 & 19.69 & 28.71 \\
\hline$i_{n}(\mathrm{deg})$. & 12.23 & 22.20 & 20.73 \\
\hline$\zeta($ deg. $)$ & 6.14 & 9.71 & 12.81 \\
\hline$\phi_{t}^{\prime}$ (deg.) & 6.15 & 8.64 & 12.61 \\
\hline$\beta_{s}^{-}$(deg.) & 23.25 & 23.25 & 23.25 \\
\hline$\beta_{s}^{+}(\operatorname{deg})$. & 1.31 & 3.65 & 4.50 \\
\hline for $r_{n}=0.5 \mathrm{~m}$ & & & \\
\hline$q_{c_{\max }}\left(\mathrm{W} / \mathrm{cm}^{2}\right)$ & 393 & 393 & 393 \\
\hline$q_{r_{\max }}\left(\mathrm{W} / \mathrm{cm}^{2}\right)$ & 78 & 78 & 78 \\
\hline for $r_{n}=1 \mathrm{~m}$ & & & \\
\hline$q_{c_{\max }}\left(\mathrm{W} / \mathrm{cm}^{2}\right)$ & 278 & 278 & 278 \\
\hline$q_{r_{\max }}\left(\mathrm{W} / \mathrm{cm}^{2}\right)$ & 114 & 114 & 114 \\
\hline
\end{tabular}



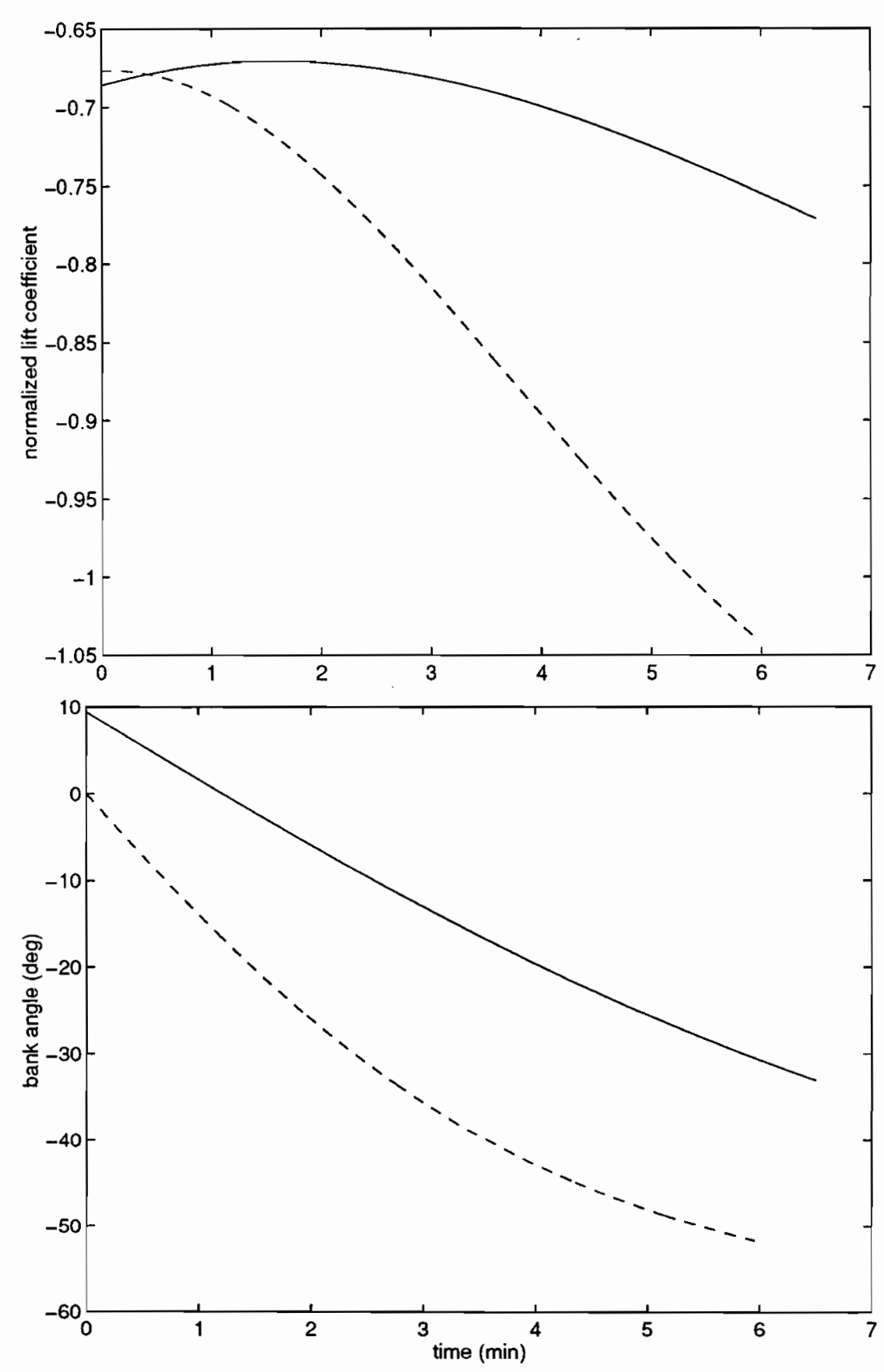

Figure 7.1: Variation of the normalized lift coefficient $\lambda$ and bank angle $\sigma$ with respect to time; — for $i_{s}=6.00 \mathrm{deg}$; - - for $i_{s}=12.00 \mathrm{deg}$. 

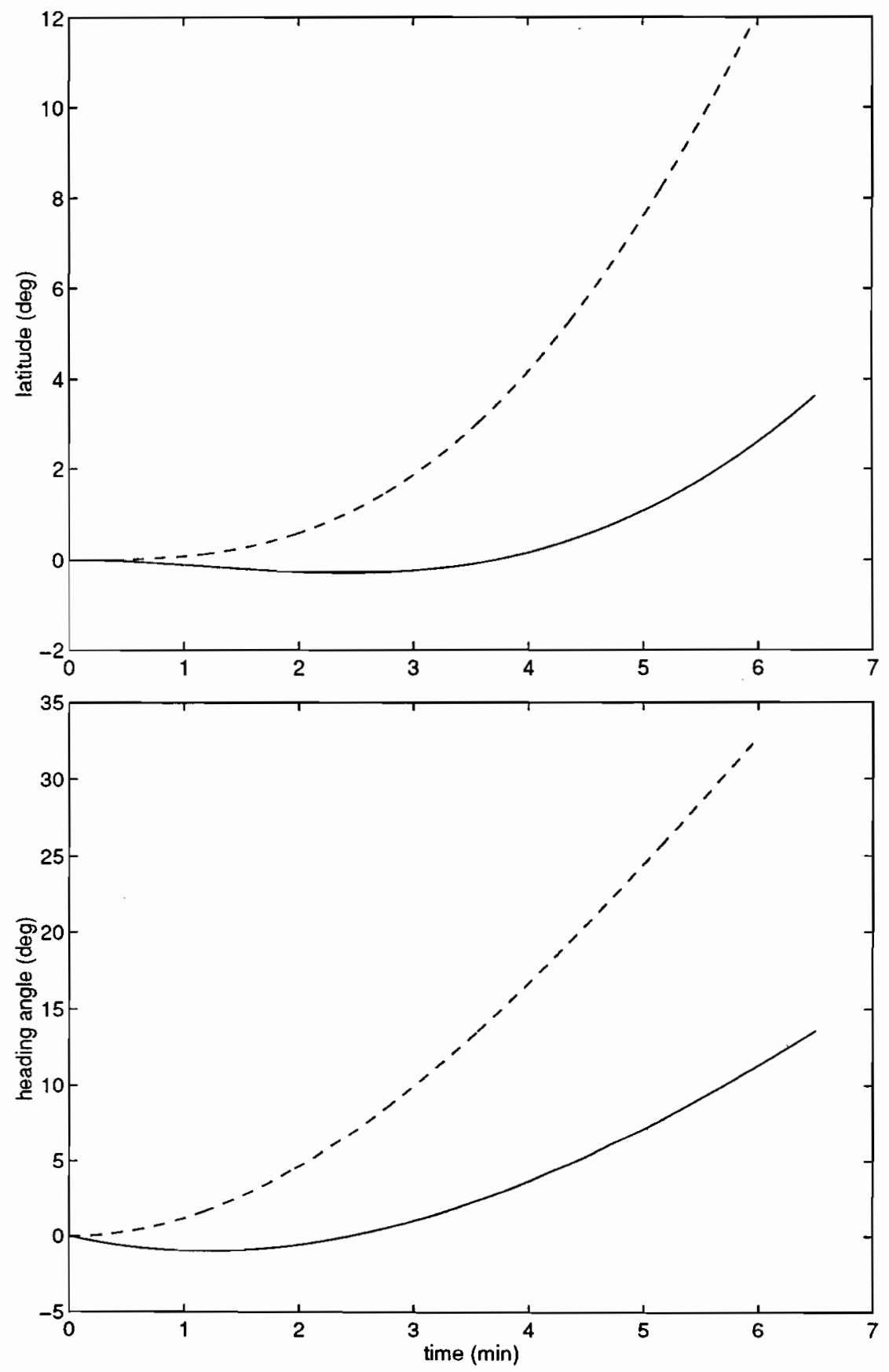

Figure 7.2: Variation of latitude $\phi$ and heading angle $\psi$ with respect to time; — for $i_{s}=6.00 \mathrm{deg} . ;-$ - for $i_{s}=12.00 \mathrm{deg}$. 


\section{Chapter 8}

\section{Conclusion}

In this thesis, we have attempted the task of studying aero-gravity assist for possible use in the future planetary and solar missions. The previous investigations were restricted to very simple analytical models only. We extended the analysis further by developing more accurate analytical models and using optimization techniques to maximize the advantage of aero-gravity assist. In the following, the conclusions of each of the chapters from Chapter 4 to 7 are given separately. Then suggestions for further study are given. Finally, original contributions of this thesis to the engineering and scientific knowledge are summarized.

In Chapter 2, we developed the objective function $V_{s}^{+}$(heliocentric velocity after aero-gravity assist) for planar and three-dimensional atmospheric trajectories. Later, in Chapter 4, the optimization problem for planar atmospheric trajectory was formulated mathematically, and the necessary conditions for the optimal atmospheric trajectory and control were derived. The results show the superiority of aero-gravity assist (AGA) over conventional gravity assist (GA) for both planets Mars and Venus; but for Venus it is superior only when $V_{\infty}^{-}$is high. To make results closer to the real situation, $n=1.5$ was considered in the drag polar. However, $V_{s}^{+}$was found to be more or less the same for both $n=2$ and 1.5 , because the trajectories in the two cases are very similar without any heating rate constraint. The heating rates were calculated, which show that increasing $C_{L}^{*}$ can reduce heating rate. Comparison of AGA from Mars and Venus revealed that Mars-AGA is more advantageous than 
Venus-AGA. In the case of Venus, AGA is better than GA only when $V_{\infty}^{-} \geq 10 \mathrm{~km} / \mathrm{s}$; the heating rates are also high. But this is not the case with Mars-AGA. The heating rates for Mars-AGA are lower than Venus-AGA, because smaller mass of Mars results in smaller $V_{e}$ for the same $V_{\infty}^{-}$, which means low heating rates. Although, $\Delta V_{E}$ (the Earth launch $\Delta V$ ) is slightly less for Venus-AGA than that for Mars-AGA, this merit of Venus-AGA vanishes when one considers the values of aphelion for the two cases. In the case of Mars-AGA, aphelion $r_{a_{s}}$ is much bigger compared to VenusAGA, because of the former's greater distance from the Sun. Hence, Mars is a better candidate for AGA than Venus.

In Chapter 5 optimal AGA results with heat constraint are given. The detailed results of AGA with heat constraint show that AGA with heat constraint gives a slightly lower heliocentric velocity $V_{s}^{+}$than that without any heat constraint, particularly when the drag polar based on the Newtonian theory for hypersonic regime is used in numerical calculations i.e, when $n=1.5$. Furthermore, the comparison of AGA and a typical aero-assisted orbit transfer (AOT) reveals that the spacecraft for an AGA mission will face somewhat higher maximum heating rate, but the time of maneuvering and the dynamic pressure in AGA will be substantially lower than that for the AOT considered. Chapter 5 also studies the effect of random fluctuation of density on the optimal $V_{s}^{+}$. It was shown that $V_{s}^{+}$is more or less unchanged if the mean density is the same; however, if the mean density is reduced to 0.6 times the normal value or less, then $V_{s}^{+}$also decreases substantially.

Chapter 6 studies the use of AGA for high energy missions (solar flyby and Pluto missions). The results show that high energy missions using only Mars-AGA are not feasible due to extremely high associated heating rates. A combination of Mars-AGA and Jupiter-GA is considered for high energy missions, which lowers heating rates and $\Delta V_{E}$ substantially. For example, the Pluto mission in 6.6 years using MAGA needs $V_{\infty_{E}}=10.3 \mathrm{~km} / \mathrm{s}$, which violates the Earth launch velocity constraint while the associated heating rate is at least $829 \mathrm{~W} / \mathrm{cm}^{2}$, which is quite high. By using MarsAGA-Jupiter-GA for a 7-year Pluto mission the Earth launch velocity and associated heating rates are reduced by $45 \%$ and $70 \%$ respectively (i.e., to $6.6 \mathrm{~km} / \mathrm{s}$ and 265 
$\mathrm{W} / \mathrm{cm}^{2}$ ). Venus-GA-Mars-AGA-Jupiter-GA can reduce $\Delta V_{E}$ further, but it may limit launch opportunities for the mission. The shielding of electronic parts from the radiation of Jupiter will not be different from the previous missions involving Jupiter gravity assist, because the closest approach considered here will be higher than most of them.

In Chapter 7, we solved the AGA problem of maximizing or minimizing $V_{s}^{+}$with heliocentric plane change. The extremely nonlinear conditions have led us to use a constant altitude approach for solving this problem. We formulated the problem for the three-dimensional atmospheric trajectory mathematically, and necessary conditions for the optimal atmospheric trajectory and control were derived. The results show that AGA with heliocentric plane change is more fuel efficient than AGA without heliocentric plane change followed by a plane change by thrust impulse. Furthermore, in the case of minimization of $V_{s}^{+}$, the fuel saving increases with the increase of the heliocentric plane change. For example, the fuel saving for $i_{s}=6^{\circ}$ is $1.07 \mathrm{~km} / \mathrm{s}$, but for $i_{s}=12^{\circ}$ the fuel saving increases to $1.45 \mathrm{~km} / \mathrm{s}$. The results also show that maximizing $V_{s}^{+}$produces smaller heliocentric plane changes compared to minimizing $V_{s}^{+}$. A small initial heliocentric inclination does not change $V_{s}^{+}$much. The results also reveal that $E^{*}$ affects the value of $V_{s}^{+}$considerably. Hence for any AGA mission, the value of $E^{*}$ will play a crucial role.

\section{Suggestions for Further Study}

In this thesis the patched conic method was used to calculate the interplanetary trajectories, which is reasonably accurate. But for calculating the transfer times this method can lead to significant errors. Therefore in future studies, where the calculation of transfer time is the main objective, more accurate methods should be used to find it (in which a three body approach should be used instead of the two body approach used in the patched conic method); particularly, for examining the launch opportunities for combined Mars-aero-gravity assist and Jupiter-gravity assist (MAGAJGA) and combined Venus-gravity assist and MAGAJGA. 
In the optimization problem, we have assumed that heating rate at only the stagnation region is of concern. But in reality, the entire leading edge might be under significant heating conditions and that needs to be studied. In the case of the moderate planetocentric velocities suggested in Chapter 6, we feel that the heating conditions will not be very severe. But if a study shows that the entire leading edge is under sever heating condition, then the total integrated heat load would also be important and active cooling will be required to keep the temperature inside the spacecraft under control.

In Chapter 7, we have restricted our analysis to only one value of $V_{\infty}^{-}$, i.e., 10 $\mathrm{km} / \mathrm{s}$. Further study is needed to examine the potential of lower or higher values of the planetocentric velocity other than $10 \mathrm{~km} / \mathrm{s}$.

Furthermore, in the case of AGA the spacecraft will maneuver at very high speed and at this speed successful control and stability will be very crucial to the mission; particularly when it encounters uncertainty in the density. Therefore, the stability and control aspects for AGA mission should be studied thoroughly. It may be pointed out that our suggested speeds for AGA are more or less of the same order as those being considered for AOT; thus on going studies on AOT will also be very helpful for the researchers working on AGA. 


\section{Original Contributions to Knowledge}

The original contributions of this study to scientific and engineering knowledge are the following:

- This is the first time that a realistic mathematical model for aerogravity assist (AGA) was presented, as opposed to calculation of some ball park figures. This is the first time that optimization techniques were used to obtain the atmospheric trajectory component of aerogravity assist (AGA).

- This is the first investigation in which the drag polar based on the Newtonian theory for hypersonic regime was used to analyze the atmospheric flight trajectory.

- This is the only study which investigates AGA with a heliocentric plane change and shows that it can lead to large fuel savings, compared with an all-propulsive maneuver.

- It was shown for the first time that combination of AGA and GA for

- high energy missions can reduce the Earth launch energy and heating rates significantly. 


\section{Appendix A}

\section{Transversality Conditions for}

\section{Planar Atmospheric Trajectory}

We have given a brief derivation of transversality conditions for planar atmospheric trajectory in Chapter 4. Here, the complete derivation of the transversality conditions are given

$$
\begin{aligned}
& p_{v_{f}}=\frac{v_{f}\left(v_{\theta} \cos \epsilon+v_{r} \sin \epsilon\right)}{v_{s} v_{\infty}^{+}}+\frac{\partial \delta}{\partial v_{f}} \frac{v_{\infty}^{+}\left(v_{\theta} \sin \epsilon-v_{r} \cos \epsilon\right)}{v_{s}}, \\
& p_{\gamma_{f}}=\frac{v_{\infty}^{+}\left(v_{\theta} \sin \epsilon-v_{r} \cos \epsilon\right)}{v_{s}} \frac{\partial \delta}{\partial \gamma_{f}} \\
& p_{\theta_{f}}=\frac{v_{\infty}^{+}\left(v_{\theta} \sin \epsilon-v_{r} \cos \epsilon\right)}{v_{s}} .
\end{aligned}
$$

First, we can write of $\partial \delta / \partial v_{f}$ as

$$
\frac{\partial \delta}{\partial v_{f}}=\frac{\partial\left(\delta_{2} / 2-\theta_{2}\right)}{\partial v_{f}},
$$

where

$$
\delta_{2} / 2=\sin ^{-1}\left(1 / e_{2}\right) .
$$

Eq. (4.6a) shows the value of $e_{2}$, and

$$
\begin{gathered}
\frac{\partial \delta_{2} / 2}{\partial v_{f}}=\frac{1}{\left(1-u_{1}\right)^{1 / 2}} \frac{\partial u_{1}}{\partial v_{f}} \text { where } u_{1}=1 / e_{2}, \\
\frac{\partial u_{1}}{\partial v_{f}}=\frac{-2 v_{f}\left(v_{f}^{2}-1\right) \cos ^{2} \gamma_{f}}{e_{2}^{3}}
\end{gathered}
$$




$$
\frac{\partial \theta_{2}}{\partial v_{f}}=\frac{1}{u^{2}+1} \frac{\partial u_{2}}{\partial v_{f}} \text { where } u_{2}=\tan \theta_{2}
$$

$\mathrm{Eq}$. (4.6b) shows the value of $\theta_{2}$, and

$$
\frac{\partial u_{2}}{\partial v_{f}}=\frac{-2 v_{f} \cos \gamma_{f} \sin \gamma_{f}}{\left(v_{f}^{2} \cos ^{2} \gamma_{f}-1\right)^{2}}
$$

Then we can write $\partial \delta / \partial \gamma_{f}$ as

$$
\frac{\partial \delta}{\partial \gamma_{f}}=\frac{\partial\left(\delta_{2} / 2-\theta_{2}\right)}{\partial \gamma_{f}}
$$

where

$$
\begin{gathered}
\frac{\partial \delta_{2} / 2}{\partial \gamma_{f}}=\frac{1}{\left(1-u_{1}\right)^{1 / 2}} \frac{\partial u_{1}}{\partial \gamma_{f}} \\
\frac{\partial u_{1}}{\partial \gamma_{f}}=\frac{\left(v_{f}^{2}-2\right) \cos \gamma_{f} \sin \gamma_{f}}{e_{2}^{3}} \\
\frac{\partial \theta_{2}}{\partial \gamma_{f}}=\frac{1}{u^{2}+1} \frac{\partial u_{2}}{\partial \gamma_{f}}, \\
\frac{\partial u_{2}}{\partial \gamma_{f}}=\frac{v_{f}^{2}\left(v_{f}^{2} \cos ^{4} \gamma_{f}+2 \sin ^{2} \gamma_{f}+v_{f}^{2} \sin ^{2} \gamma_{f} \cos ^{2} \gamma_{f}-1\right)}{\left(v_{f}^{2} \cos ^{2} \gamma_{f}-1\right)^{2}}
\end{gathered}
$$

Finally, we have $\partial \delta / \partial \theta_{f}=1$. 


\section{Appendix B}

\section{Transversality Conditions for 3-Dimensional Atmospheric}

\section{Trajectory}

In Chapter 7 , we have given the transversality conditions briefly, the complete derivation of them are given now. For the case of maximization of $V_{s}^{+}$, the transversality conditions are as follows:

$$
\begin{aligned}
& \frac{\partial J}{\partial \theta_{f}}=\frac{v_{\infty}^{+}\left(v_{\theta_{s}} \sin \epsilon-v_{r_{s}} \cos \epsilon\right)}{v_{s}^{+}} \frac{\partial \alpha_{t}}{\partial \theta_{f}}, \\
& \frac{\partial J}{\partial \phi_{f}}=\frac{v_{\infty}^{+}\left(v_{\theta_{s}} \sin \epsilon-v_{r_{s}} \cos \epsilon\right)}{v_{s}^{+}} \frac{\partial \alpha_{t}}{\partial \phi_{f}} \\
& \frac{\partial J}{\partial \psi_{f}}=\frac{v_{\infty}^{+}\left(v_{\theta_{s}} \sin \epsilon-v_{r_{s}} \cos \epsilon\right)}{v_{s}^{+}} \frac{\partial \alpha_{t}}{\partial \psi_{f}}, \\
& \frac{\partial J}{\partial v_{f}}=\frac{v_{f}\left(v_{\theta_{s}} \cos \epsilon+v_{r_{s}} \sin \epsilon\right)}{v_{s}^{+} v_{\infty}^{+}}+\frac{\partial \alpha_{t}}{\partial v_{f}} \frac{v_{\infty}^{+}\left(v_{\theta_{s}} \sin \epsilon-v_{r_{s}} \cos \epsilon\right)}{v_{s}^{+}} .
\end{aligned}
$$

For the case of minimization of $V_{s}^{+}$, the above equations become

$$
\begin{aligned}
& \frac{\partial J}{\partial \theta_{f}}=\frac{-v_{\infty}^{+}\left(v_{\theta_{s}} \sin \epsilon+v_{r_{s}} \cos \epsilon\right)}{v_{s}^{+}} \frac{\partial \alpha_{t}}{\partial \theta_{f}} \\
& \frac{\partial J}{\partial \phi_{f}}=\frac{-v_{\infty}^{+}\left(v_{\theta_{s}} \sin \epsilon+v_{r_{s}} \cos \epsilon\right)}{v_{s}^{+}} \frac{\partial \alpha_{t}}{\partial \phi_{f}} \\
& \frac{\partial J}{\partial \psi_{f}}=\frac{-v_{\infty}^{+}\left(v_{\theta_{s}} \sin \epsilon+v_{r_{s}} \cos \epsilon\right)}{v_{s}^{+}} \frac{\partial \alpha_{t}}{\partial \psi_{f}} \\
& \frac{\partial J}{\partial v_{f}}=\frac{-v_{f}\left(-v_{\theta_{s}} \cos \epsilon+v_{r_{s}} \sin \epsilon\right)}{v_{s}^{+} v_{\infty}^{+}}-\frac{\partial \alpha_{t}}{\partial v_{f}} \frac{v_{\infty}^{+}\left(v_{\theta_{s}} \sin \epsilon+v_{r_{s}} \cos \epsilon\right)}{v_{s}^{+}}
\end{aligned}
$$


For $p_{\theta_{f}}$, we have

$$
\frac{\partial \alpha_{t}}{\partial \theta_{f}}=P_{1} \frac{\partial u_{3}}{\partial \theta_{f}}
$$

and

$$
\begin{aligned}
P_{1} & =\frac{-1}{\left(1-u_{3}^{2}\right)^{1 / 2}}, \\
u_{3} & =\cos \theta_{t} \cos \phi_{t}, \\
\frac{\partial u_{3}}{\partial \theta_{f}} & =-\cos \phi_{t} \sin \theta_{t},
\end{aligned}
$$

as we have $\partial \theta_{t} / \partial \theta_{f}=1$ for the maximizing $V_{s}^{+}$case while for the minimizing $V_{s}^{+}$case $\partial \theta_{t} / \partial \theta_{f}=-1$

For $p_{\phi_{f}}$, we have

$$
\frac{\partial \alpha_{t}}{\partial \phi_{f}}=P_{1} \frac{\partial u_{3}}{\partial \phi_{f}}
$$

where

$$
\frac{\partial u_{3}}{\partial \phi_{f}}=-P_{2} \cos \theta_{t} \sin \phi_{t} \frac{\partial u_{4}}{\partial \phi_{f}}-\cos \phi_{t} \sin \theta_{t}\left(P_{3} \frac{\partial u_{5}}{\partial \phi_{f}}-\frac{\partial \Omega}{\partial \phi_{f}}\right)
$$

and

$$
\begin{aligned}
P_{2} & =\frac{1}{\left(1-u_{4}\right)^{1 / 2}}, \\
P_{3} & =\frac{1}{1+u_{5}^{2}}, \\
u_{4} & =\sin i \sin \Lambda^{\prime}, \\
\Lambda^{\prime} & =\pi / 2+u+\delta_{2} / 2, \\
\delta_{2} & =2 \sin \left(\frac{1}{e_{2 c}}\right),
\end{aligned}
$$

( $e_{2}$, is the eccentricity of $H_{T_{2}}$ in the case of constant altitude during maneuvering through the atmosphere and is defined as $e_{2_{c}}=v_{f}^{2}-1$.)

$$
\begin{aligned}
u_{5} & =\tan \Lambda \cos i \\
\frac{\partial u_{4}}{\partial \phi_{f}} & =P_{4} \cos i \sin \Lambda^{\prime} \sin \phi_{f} \cos \psi_{f}+\sin i \cos \Lambda^{\prime} \frac{\partial u}{\partial \phi_{f}} \\
\frac{\partial u_{5}}{\partial \phi_{f}} & =-\tan (\Lambda) \cos \psi_{f} \sin \phi_{f}+\cos i \sec ^{2} \Lambda \frac{\partial u}{\partial \phi_{f}} \\
\Lambda & =u+\delta_{2} / 2
\end{aligned}
$$




$$
\begin{aligned}
\frac{\partial u}{\partial \phi_{f}} & =P_{4} \frac{\partial u_{6}}{\partial \phi_{f}} \\
P_{4} & =\frac{-1}{\left(1-u_{6}^{2}\right)^{1 / 2}} \\
u_{6} & =\cos \Omega \cos \phi_{f}, \\
\frac{\partial u_{6}}{\partial \phi_{f}} & =-\cos \phi_{f} \sin \Omega \frac{\partial \Omega}{\partial \phi_{f}}-\sin \phi_{f} \cos \Omega \\
\frac{\partial \Omega}{\partial \phi_{f}} & =\frac{\cos i \sin ^{2} i-\sin ^{2} \phi_{f} \cos \phi_{f} \cos \psi_{f}}{\sin ^{3} i \cos \Omega} .
\end{aligned}
$$

For $p_{v_{f}}$, we have

$$
\frac{\partial \alpha_{t}}{\partial v_{f}}=P_{1} \frac{\partial u_{3}}{\partial v_{f}}
$$

where

$$
\frac{\partial u_{3}}{\partial v_{f}}=-P_{2} \cos \theta_{t} \sin \phi_{t} \frac{\partial u_{4}}{\partial v_{f}}-\cos \phi_{t} \sin \theta_{t}\left(P_{3} \frac{\partial u_{5}}{\partial v_{f}}\right)
$$

and

$$
\begin{aligned}
\frac{\partial u_{4}}{\partial v_{f}} & =\sin i \cos \Lambda^{\prime} \frac{\partial\left(\delta_{2} / 2\right)}{\partial v_{f}}, \\
\frac{\partial u_{5}}{\partial v_{f}} & =\cos i \sec ^{2} \Lambda \frac{\partial\left(\delta_{2} / 2\right)}{\partial v_{f}} \\
\frac{\partial\left(\delta_{2} / 2\right)}{\partial v_{f}} & =\frac{-2 v_{f}\left(v_{f}^{2}-1\right)}{e_{2}^{3}\left[1-\left(1 / e_{2}\right)^{2}\right]^{1 / 2}} .
\end{aligned}
$$

For $p_{\psi_{f}}$, we have

$$
\frac{\partial \alpha_{t}}{\partial \psi_{f}}=P_{1} \frac{\partial u_{3}}{\partial \psi_{f}}
$$

where

$$
\frac{\partial u_{3}}{\partial \psi_{f}}=-P_{2} \cos \theta_{t} \sin \phi_{t} \frac{\partial u_{4}}{\partial \psi_{f}}-\cos \phi_{t} \sin \theta_{t}\left(P_{3} \frac{\partial u_{5}}{\partial \psi_{f}}-\frac{\partial \Omega}{\partial \psi_{f}}\right)
$$

and

$$
\begin{aligned}
\frac{\partial u_{4}}{\partial \psi_{f}} & =P_{4} \cos i \sin \Lambda^{\prime} \sin \psi_{f} \cos \phi_{f}+\sin i \cos \Lambda^{\prime} \frac{\partial u}{\partial \psi_{f}} \\
\frac{\partial u_{5}}{\partial \psi_{f}} & =-\tan \Lambda \cos \phi_{f} \sin \psi_{f}+\cos i \sec ^{2} \Lambda \frac{\partial u}{\partial \psi_{f}} \\
\frac{\partial u}{\partial \psi_{f}} & =P_{4} \frac{\partial u_{6}}{\partial \psi_{f}}
\end{aligned}
$$




$$
\begin{aligned}
\frac{\partial u_{6}}{\partial \psi_{f}} & =-\cos \phi_{f} \sin \Omega \frac{\partial \Omega}{\partial \phi_{f}} \\
\frac{\partial \Omega}{\partial \phi_{f}} & =\frac{-\sin \phi_{f} \sin \psi_{f}}{\cos \Omega \sin ^{3} i}
\end{aligned}
$$




\section{Bibliography}

[1] J. D. Anderson, et al., Hypersonic Waveriders for Planertary Atmospheres, Journal of pacecraft and Rockets, Vol.28, No.4, July-August, 1991, pp. 401-410.

[2] J. D. Anderson, Hypersonic and High Temperature Gas Dynamics, McGraw-Hill, New York, 1989.

[3] U. M. Ascher, R. M. M. Mattheij and R. D. Russel, Numerical Solution of Bounday Value Problems for Ordinary Differential Equations, Prentice Hall, Englewood Cliffs, New Jersey, 1988.

[4] R. H. Battin, An Introduction to the Mathematics and Methods of Astrodynamics, American Institute of Aeronautics and Astronautics Inc., New York, 1987.

[5] J. S. Chern, C. Y. Yang, N. X. Vinh and J. M. Hanson, Deceleration and Heating Constrained Footprint of Shuttle Vehicles, Acta Astranautica, Vol. 12, No. 10, 1985, pp. 819-829.

[6] S. D. Conte and Carle de Boor, Elementary Numerical Analysis, McGraw-Hill, New York, 1980.

[7] E. Cuadra and P. D. Arther, Orbit Plane Change by External Burning Aerocruise, Journal of Spacecraft and Rockets, Vol. 3, No. 3, 1966, pp. 347-352.

[8] L. A. D'Amario, D. V. Byrnes, J. R. Johannesen, and B. G. Nolan, Galileo 1989 VEEGA Trajectory Design, Journal of the the Astronautical Sciences, Vol. 37, No. 3, July-September 1989, pp. 281-306. 
[9] J. M. Deerwester, Jupiter Swingby Missions to the Outer Planet, Journal of Spacecraft and Rockets, Vol. 3, No. 10, 1966, pp. 1564-1567.

[10] R. Deutsch, Orbital Dynamics of Space Vehicles, Prentice-Hall, New Jersey, 1963.

[11] R. Diehl, et al., Low Launch Energy Trajectories to the Outer Solar System via Venus and Earth Gravity-Assist Flybys, Advances in the the Astronautical Sciences, Vol. 65, Part I, 1987, pp. 191-206.

[12] M. W. Hunter II, Future Unmanned Exploration of the Solar System, Astronautics Aeronautics, Vol. 2, 1964, pp. 16-26.

[13] M. H. Kaplan, Modern Spacecraft Dynamics and Control, pp. 92-96, John Wiley and Sons, New York, 1976.

[14] K. Y. Kondratyev and G. E. Hunt, Weather and Climate on Planets, Pergamon Press, Oxford, 1982, pp. 406-409.

[15] B. Lee and W. J. Grantham, Aeroassisted Orbital Maneuvering Using Lyapunove Optimal Feedback Control, Journal of Guidance, Control and Dynamics, Vol. 12, No. 2, 1989, pp. 237-242.

[16] M. J. Lewis and A. P. Kothari, Hypersonic Waverider for Planetary Maneuvering, presented at 1st International Hypersonic Waverider Symposium, University of Maryland, College Park, Maryland, Oct. 17-19, 1990.

[17] M. J. Lewis and A. D. McRonald, Design of Hypersonic Waveriders for Aeroassisted Interplanetary Trajectories, Journal of Spacecraft and Rockets, Vol. 29, No. 5,1992 , pp. 653-660.

[18] H. S. London, Change of Satellite Orbit Plane by Aerodynamic Maneuvering, Journal of the Aerospace Sciences, Vol. 29, March 1962, pp. 323-332.

[19] I. McCausland, Introduction to Optimal Control, Wiley, New York, 1969. 
[20] W. I. McLaughlin, Ulysses Swings by Jupiter, Spaceflight, Vol. 34, 1992, pp. 166-167.

[21] A. D. McRonald and J. E. Randolph, Hypersonic Maneuvering to Provide Planetary Gravity Assist, Journal of Spacecraft and Rockets, Vol. 29, No. 2, MarchApril, 1992, pp. 216-222.

[22] K. D. Mease, Optimization of Aeroassisted Orbital Transfer: Current Status, Journal of the the Astronautical Sciences, Vol. 36, No. 1/2, 1988, pp. 7-33.

[23] K. D. Mease, J. Y. Lee and N. X. Vinh, Orbital Changes during Hypersonic Aerocruise, Journal of the Astronautical Sciences, Vol. 36, No. 1, Jan.-Jun. 1988, pp 103-137.

[24] K. D. Mease and N. X. Vinh, Minimum Fuel Aero-assisted Coplanar Orbital Transfer Using Lift Modulation, Journal of Guidance and Control, Vol. 8, No. 1, Jan.-Feb. 1985, pp 134-141.

[25] K. D. Mease, N. X. Vinh and S. H. Kuo, Optimal Plane Change During Constant Altitude Hypersonic Flight, Journal of Guidance, Control and Dynamics, Vol. 14, No. 4, Jul.-Aug. 1991, pp 797-806.

[26] S. Medepalli and N. X. Vinh, Optimal Plane Change of an Elliptic Orbit During Aerocruise, Journal of the Astronautical Sciences, Vol. 40, No. 4, 1992, pp. 503525.

[27] A. Miele, Flight Mechanics Vol. 1, Theory of Flight Paths, Addison-Wesley Publishing Company, Inc., 1966.

[28] A. Miele and V. K. Basapur, Approximate Solutions of Minimax Optimal Control Problems for Aeroassisted Orbital Transfer, Acta Astranautica, Vol. 12, No. 10, 1985, pp. 808-819.

[29] A. Miele, V. K. Basapur and K. D. Mease, Nearly-Grazing Optimal Trajectories for Aeroassisted Orbital Transfer, Journal of the Astronautical Sciences, Vol. 34, No. 1, 1986, pp. 3-18. 
[30] A. Miele, V. K. Basapur and W. Y. Lee, Optimal Trajectories for Aeroassisted Coplanar Orbital Transfer, Journal of Optimization Theory and Applications, Vol. 52, No. 1, 1987, pp. 1-24.

[31] A. Miele, V. K. Basapur and W. Y. Lee, Optimal Trajectories for Aeroassisted, Noncoplanar Orbital Transfer Part 1, Acta Astranautica, Vol. 15, No. 6/7, 1987, pp. 399-411.

[32] A. Miele, W. Y. Lee, and K. D. Mease, Optimal Trajectories for Aeroassisted, Non-coplanar Orbital Transfer, Part 2: HEO-to-LEO Transfer, Paper No. IAF87-328, 38th Congress of the International Astronautical Federation, Brighton, England, 1987.

[33] A. Miele and P. Venkataraman, Optimal Trajectories for Aeroassisted Orbital Transfer, Acta Astranautica, Vol. 11, Nos. 7-8, 1984, pp. 423-433.

[34] J. C. Niehoff, Gravity-Assisted Trajectory to Solar System, Journal of Spacecraft and Rockets, Vol. 3, No. 9, 1966, pp. 1351-1356.

[35] W. A. Page and H. T. Woodward, Radiative and Convective Heating During Venus Entry, AIAA Journal, Vol.10, No.10, 1972, pp. 1379-1381.

[36] J. A. Penlund, Wall Temperature Effects on the Aerodynamics of a HydrogenFueled Transport Concept in Mach 8 Blowdown and Shock Tunnels, NASA TP 2159, July, 1983.

[37] L. S. Pontryagin, et al., The Mathematical Theory of Optimal Processes, Wiley Interscience, New York, 1962.

[38] J. E. Randolph and A. D. McRonald, Solar Probe Mission Status, AAS Paper 89-212, presented at the AAS/GSFC International Symposium on Orbital Mechanics and Mission Design, Washington D.C., 24-27 April, 1989.

[39] M. Rycroft, The Cambridge Encyclopedia of Space, Cambridge University Press, UK, 1990. 
[40] Alvin Seiff, Atmospheres of Earth, Mars and Venus, as Defined by Entry Probe Experiments, Journal of Spacecraft and Rockets, Vol.28, No.3, May-June, 1991, pp. 265-275.

[41] R. S. Shevell, Fundamentals of Flight, Printice-Hall, Englewood Cliffs, New Jersey, 1989.

[42] J. Stoer and R. Bulirsch, Introduction to Numerical Analysis, Springer-Verlag, New York, 1980.

[43] K. Sutton and R. A. Graves, Jr., A General Stagnation Point Convective Heating Equation for Arbitrary Gas Mixtures, NASA TR R-376, Nov., 1971.

[44] M. E. Tauber and K. Sutton, Stagnation-Point Radiative Heating Relations for Earth and Mars Entries, Journal of Spacecraft and Rockets, Vol.28, 1991, pp. 40-42.

[45] N. X. Vinh, A. Buseman and R. D. Culp, Hypersonic and Planetary Entry Flight Mechanics, The University of Michigan Press, Ann Arbor, Michigan, 1980.

[46] N. X. Vinh, Optimal Trajectories in Atmospheric Flight, Elsevier Scientific Publishing Company, New York, 1981.

[47] N. X. Vinh, Flight Mechanics of High Performance Aircraft, Cambridge University Press, Cambridge, UK, 1993.

[48] N. X. Vinh and J. M. Hanson, Optimal Aeroassited Return from High Earth Orbit with Plane Change, Acta Astranautica, Vol. 12, No. 1, 1985, pp. 11-25.

[49] N. X. Vinh and J. R. Johannesen, Optimal Aeroassited Transfer between Coplanar Elliptical Orbits, Acta Astranautica, Vol. 13, No.6/7, 1986, pp. 291-299.

[50] G. D. Walberg, A Survey of Aero-assisted Orbit Transfer, Journal of Spacecraft and Rockets, Vol. 22, No. 1, Jan.-Feb., 1985, pp. 3-18.

[51] S. Yakowitz and F. Szidarovszky, An Introduction to Numerical Computations, Macmillan, New York, 1986. 